\title{
Field-Portable Dissolved Gas Sensing and Perspectives in Aqueous Microplastic Detection
}

\author{
by
}

Morgan Grace Blevins

B.S., Harvey Mudd College (2019)

Submitted to the Department of Aeronautics and Astronautics

in partial fulfillment of the requirements for the degree of

Master of Science in Aeronautics and Astronautics

at the

MASSACHUSETTS INSTITUTE OF TECHNOLOGY AND WOODS HOLE OCEANOGRAPHIC INSTITUTION

June 2021

(C) 2021 Massachusetts Institute of Technology and Woods Hole Oceanographic Institution. All rights reserved.

The author hereby grants to MIT and WHOI permission to reproduce and to distribute publicly paper and electronic copies of this thesis document in whole or in part in any medium now known or hereafter created.

Author

Department of Aeronautics and Astronautics

May 18, 2021

Certified by

Anna P. M. Michel

Associate Scientist of Applied Ocean Physics and Engineering, WHOI Thesis Supervisor

Accepted by

Zoltan Spakovszky

Professor of Aeronautics and Astronautics Chair, Graduate Program Committee

Accepted by

David Ralston Associate Scientist with Tenure of Applied Ocean Physics and Engineering Chair, Joint Committee on Applied Ocean Science and Engineering 


\title{
Field-Portable Dissolved Gas Sensing and Perspectives in Aqueous Microplastic Detection
}

\author{
by \\ Morgan Grace Blevins \\ Submitted to the Department of Aeronautics and Astronautics \\ on May 18, 2021, in partial fulfillment of the \\ requirements for the degree of \\ Master of Science in Aeronautics and Astronautics
}

\begin{abstract}
Global temperature rise and increased atmospheric carbon dioxide $\left(\mathrm{CO}_{2}\right)$ levels have affected the health of the world's ocean and water ecosystems, impacting the balances of natural carbon cycling and causing ocean acidification. Additionally, as global temperatures rise, thawing permafrost has stimulated increased release of methane $\left(\mathrm{CH}_{4}\right)$, a gas with a shorter lifetime in the atmosphere but with even more heat trapping ability than $\mathrm{CO}_{2}$. In situ analysis of dissolved gas content in surface waters is currently performed with large, expensive instruments, such as spectrometers, which are coupled with gas equilibration systems, which extract dissolved gas from water and feed it to the sensor. Accurate, low cost, and portable sensors are needed to measure the dissolved $\mathrm{CH}_{4}$ and $\mathrm{CO}_{2}$ concentration in water systems to quantify their release and understand their relationship to the global carbon budget. At the same time, while greenhouse gases are well established threats to water ecosystems, the ubiquity and potential consequences of microplastics in aqueous environments are just beginning to be recognized by the environmental research community. Microplastics (MPs) are small particles of polymer debris, commonly defined as being between $1 \mu \mathrm{m}$ and $1000 \mu \mathrm{m}$. Despite the pervasiveness of MPs, our ability to characterize MPs in the environment is limited by the lack of technologies for rapidly and accurately identifying and quantifying MPs. This thesis is concerned with the engineering challenges prompted by the need for high quality and quantity environmental data to better study and the impact, cycling, and prevalence of these pollutants in aqueous environments.

Three distinct investigations are presented here. First, the design of the LowCost Gas Extraction and Measurement System (LC-GEMS) for dissolved $\mathrm{CO}_{2}$ is presented. At just under $\$ 600$ dollar to build, the LC-GEMS is an ultra-portable, toolbox-sized instrument for dissolved gas sensing in near-surface waters. The LCGEMS was characterized in the lab and demonstrated linear relationships with dissolved $\mathrm{CO}_{2}$ as well as temperature. Lab calibrations and subsequent field testing in the Little Sippewissett Marsh, in Falmouth, Massachusetts showed that the LCGEMS captures both diurnal and minute-time scale trends in dissolved $\mathrm{CO}_{2}$.
\end{abstract}


Second, this thesis presents the novel design of three simple and low-cost planar nanophotonic and plasmonic structures as optical transducers for measuring dissolved $\mathrm{CH}_{4}$. Through simulations, the sensitivity of the structures are evaluated and found to exhibit superior performance in the reflectance intensity readout mode to that of the standard surface-plasmon-polariton-mode Spreeta sensor. A practical, small, and low-cost implementation of this chip with a simple intensity-based measurement scheme is proposed. This design is novel in the space of dissolved gas monitoring because it shows potential to measure directly in the water phase while being robust and low-cost to implement.

Finally, this thesis presents a literature review and perspective to motivate the development of field-deployable microplastic sensing techniques. A framework for field-deployable microplastic sensing is presented and seeks to inform the MP community of the potential in both traditional MP analysis techniques and unconventional methods for creating rapid and automated MP sensors. The field-deployabilty framework addresses a full scope of practical/technological trade-offs to be considered for portable MP detection.

Thesis Supervisor: Anna P. M. Michel

Title: Associate Scientist of Applied Ocean Physics and Engineering, WHOI 


\section{Acknowledgments}

Firstly, I extend my gratitude to my advisor, Dr. Anna Michel, for her mentorship, insights into the world of ocean instrumentation, and example as a scientist and engineer. I truly appreciate all that I have learned and experienced as a member of WHOI's Chemical Sensing Laboratory as well as all of the incredible scientists and engineers I have have the pleasure to work with at WHOI and MIT. To my labmates Beckett and Victoria, I give thanks for their support, thoughtful feedback, and collaboration. I also thank Sarah Youngs for her crucial support in conducting fieldwork for this thesis and getting stuck in the mud with me. In addition, I would like to acknowledge the Wampanoag Nation, made up of the Mashpee, Aquinnah, Herring Pond Tribes, whose ancestors are the original inhabitants of present day Massachusetts and Cape Cod where I have conducted by research and fieldwork for this thesis.

I acknowledge and thank the Karen L. Von Damm Graduate Student Fellowship and the Draper Fellow Program for funding my graduate studies. I would also like to extend my thanks to Dr. Mark Wikinski for his mentorship as my Draper Fellowship advisor and Dr. Louis Kratchman for providing his advisement on field-portable microplastic analysis. For their technical collaborations and insights in this thesis work, I also thank Dr. Svetlana Boriskina, Jason Kapit, and Shawnee Traylor.

I thank my partner, Charles, for all of the miles run around the Charles River and Fells which were essential to finding balance in graduate school. To my mom, Linda, I owe thanks for always rooting for me and supporting me in my endeavors and dreams. Finally, I am grateful to my dad, Earl, and brother, Quentin, for providing endless inspiration. 


\section{Contents}

1 Introduction $\quad 15$

1.1 Background on Greenhouse Gases and Aqueous Environments . . . 16

1.1.1 Measuring the carbon cycle in aqueous environments . . . . 16

1.2 Background on In Situ and Field-Deployable Aqueous Sensing Tech-

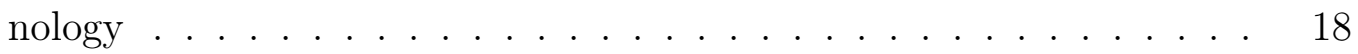

1.2.1 Gas Extraction and Equilibration . . . . . . . . . . . . 19

1.2 .2 Gas Analysis . . . . . . . . . . . . . . . . . . . 23

\section{A Low-Cost Gas Extraction and Measurement System (LC-GEMS)} for dissolved gas analysis in aqueous environments 25

2.1 Introduction . . . . . . . . . . . . . . . 25

$2.2 \mathrm{pCO}_{2}$ in salt marshes $\ldots \ldots \ldots \ldots \ldots \ldots \ldots \ldots$

2.2.1 Measuring dissolved $\mathrm{CO}_{2}$ in aqueous environments $\left(\mathrm{pCO}_{2}\right) \ldots 26$

2.2.2 State of the art in dissolved $\mathrm{CO}_{2}$ sampling and analysis . . . 27

2.3 Design of the Low-Cost Gas Extraction and Measurement System (LC-

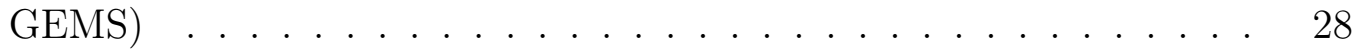

2.3 .1 Gas Extraction System . . . . . . . . . . . . . . . . . . . 29

2.3 .2 Sensor Payload . . . . . . . . . . . . . . . . . . . . 34

2.3.3 Portable Power System . . . . . . . . . . . . . . . . . . . . . 39

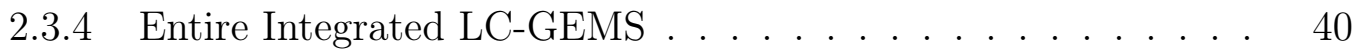

$2.4 \mathrm{~K}^{2} \mathrm{CO}_{2}$ Sensor Calibrations . . . . . . . . . . . . . 40

2.5 LC-GEMS Calibrations and Characterization . . . . . . . . . . 42

2.5.1 LC-GEMS Gas Extraction Calibration . . . . . . . . . 42 
2.5.2 LC-GEMS Time Response . . . . . . . . . . . . . . . . 51

2.6 Field Measurements . . . . . . . . . . . . . . . . . . . . . 53

2.6 .1 Field site $\ldots \ldots \ldots \ldots \ldots \ldots \ldots \ldots$

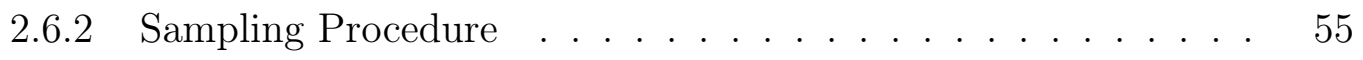

2.6.3 Field Site Measurement Results _ . . . . . . . . . . . . 56

2.6.4 Discussion of Field Performance . . . . . . . . . . . . . 62

2.6 .5 Conclusion . . . . . . . . . . . . . . . . . . 64

3 Planar nanophotonic structures for intensity based readout refractive index sensing applied to dissolved methane detection $\quad 67$

3.1 The Motivation for a Photonic Approach to Methane Sensing . . . . 68

3.2 Methods . . . . . . . . . . . . . . . . . . . . 71

3.2.1 Reflectance Intensity based readout mode . . . . . . . . . 71

3.2.2 Design of planar nanophotonic structures for reflectance intensity based readout . . . . . . . . . . . . . 73

3.2.3 Reference Surface Plasmon Resonance (SPR) sensor . . . . . . 74

3.2.4 Bragg reflector with a single defect layer (Bragg defect structure) 75

3.2.5 Multi-layer stack that supports Tamm plasmon mode (Tamm structure $\ldots \ldots \ldots \ldots \ldots \ldots \ldots \ldots$

3.2.6 Hybrid multi-layer stack that acts as a magnetic mirror (Mag-

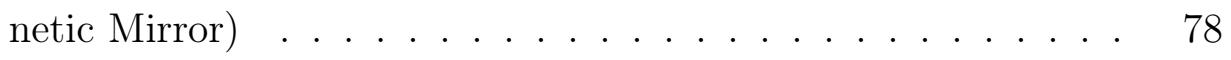

3.2.7 Simulation Methodology _. . . . . . . . . . . . . . . 79

3.3 Results and Discussion . . . . . . . . . . . . . . . 80

3.3.1 Comparable performance of the planar sensor structures . . . 80

3.3.2 Measuring both polarizations for sensitivity enhancement . . . 84

3.3.3 Discussion of chip robustness in seawater . . . . . . . . 86

3.3 .4 Conclusion . . . . . . . . . . . . . . . . . . 88

4 Perspectives on Field-Deployable Microplastic Sensing $\quad 91$

4.1 Introduction . . . . . . . . . . . . . . . . 91

4.2 Framework of Field-Deployable Microplastic Sensing . . . . . . . . . 93 
4.2.1 Field-Deployability Tradespace . . . . . . . . . . . . . . 94

4.2.2 Principles of Operation of MP Measurement Techniques and their Data Products . . . . . . . . . . . . . . . . 94

4.3 Review Results . . . . . . . . . . . . . . . . . . . . . . . 96

4.4 Discussion . . . . . . . . . . . . . . . . . . . 98

$\begin{array}{lll}5 & \text { Conclusion } & 101\end{array}$

5.1 The LC-GEMS for $\mathrm{pCO}_{2}$ Sensing . . . . . . . . . . . . . . . . 101

5.2 Planar Nanophotonic Chips for dissolved $\mathrm{CH}_{4}$ Sensing . . . . . . . . . 102

5.3 Field Portable Microplastic Sensing . . . . . . . . . . . . . . . . 102

5.4 Final words . . . . . . . . . . . . . . . . . . . . 103

$\begin{array}{ll}\text { Bibliography } & 104\end{array}$ 


\section{List of Figures}

1-1 A CTD rosette being recovered on a National Oceanic and Atmospheric Administration (NOAA) ship . . . . . . . . . . . . . 19

1-2 Illustration of headspace equilibration . . . . . . . . . . . . . 20

2-1 System diagram of the LC-GEMS . . . . . . . . . . . . . . 29

2-2 Diagram of gas extraction system of the LC-GEMS . . . . . . . . . . 32

2-3 Photos of gas extraction system of the LC-GEMS . . . . . . . . . 33

2-4 Diagram of sensor payload system of the LC-GEMS . . . . . . . . . . 34

2-5 Photos of sensor payload system of the LC-GEMS . . . . . . . . . 38

2-6 Diagram of power routing system of the LC-GEMS . . . . . . . . . 39

2-7 Photos of the full LC-GEMS system, front and back, in the field. . . . 40

2-8 Multivariate linear regression model for $\mathrm{K}^{2} \mathrm{CO}_{2}$ sensors . . . . . . . 43

2-9 The showerhead equilibrator set-up for LC-GEMS calibration . . . . 44

2-10 The temperature control system for lab calibration of LC-GEMS . . . 44

2-11 LI-COR showerhead equilibration data . . . . . . . . . 45

2-12 Raw extraction calibration measurements for the LC-GEMS . . . . . 47

2-13 Gas extraction calibration plots for LC-GEMS for fresh and saltwater with multivariate linear regression fits . . . . . . . . . . . . . 49

2-14 Confidence and prediction intervals for LC-GEMS . . . . . . . . . 50

2-15 LC-GEMS step response and steady-state reading . . . . . . . . . 51

2-16 Satellite imagery of the Little Sippewissett Marsh field testing site . . 54

2-17 Photographs of the Little Sippewissett Marsh field testing site. . . . . 54

2-18 LC-GEMS making measurement in the field along side reference sensors. 55 
2-19 Turner Designs C-sense reference $\mathrm{CO}_{2}$ sensor and RBRconcerto CTD $\quad 57$

2-20 Field testing results of LC-GEMS, April 19th and 20th, 2021 . . . . 58

2-21 Field testing results of LC-GEMS, April 28th . . . . . . . . . . 59

2-22 Field testing results, 12:40 April 28th, 2021 hour long LC-GEMS measurement . . . . . . . . . . . . . . . . 60

2-23 Measurement agreement scatter plot of LC-GEMS and C-sense $\mathrm{CO}_{2}$ readings ......................... 63

3-1 Working Principle of Cryptophane-A in Methane Sensing . . . . . . . 69

3-2 Intensity-based read of a photonic refractive index sensing structure . 72

3-3 Design, resonance condition, and electric field distribution of a SPR

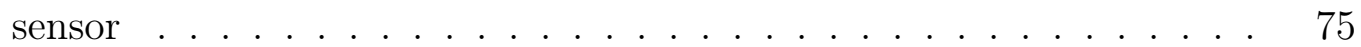

3-4 Design, resonance condition, and electric field distribution of a Bragg defect sensor . . . . . . . . . . . . . . . . . . 76

3-5 Design, resonance condition, and electric field distribution of a Tamm plasmon sensor . . . . . . . . . . . . . . . . . . . 78

3-6 Design, resonance condition, and electric field distribution of a magnetic mirror sensor . . . . . . . . . . . . . . . . . . . . . . . 79

3-7 Reflectance spectra for Bragg defect, Tamm Plasmon, and magnetic mirror sensor configurations and for the reference SPR sensor . . . . . 81

3-8 Angular shift of the resonant dip for each sensing structure . . . . . . 82

3-9 Intensity based readout of each sensing structure. . . . . . . . . . . . 84

3-10 The s and p-polarization reflectance profiles of the Bragg Defect structure and their ratios. . . . . . . . . . . . . . . 85

3-11 Schematic of a reflectance intensity-based readout implementation of the Bragg defect structure and its expected readout. . . . . . . . . . 89

4-1 The framework of field-portable MP sensing techniques: Key data products and characteristics . . . . . . . . . . . . . 95 


\section{List of Tables}

1.1 Carbon metrics for ocean and aqueous analysis . . . . . . . . . . . 17

2.1 Differences between the FaRAGE gas extraction system and the LCGEMS gas extration stage . . . . . . . . . . . . . . . 31

2.2 Parts list and cost of the LC-GEMS . . . . . . . . . . . . . . . . . 41

2.3 Gas extraction time constants, $t_{63 \%}$, of the LC-GEMS . . . . . . . . . 52

3.1 Performance metrics for each RI sensing structure . . . . . . . . 83

4.1 Microplastic sensing techniques . . . . . . . . . . . . . 96

4.1 Microplastic sensing techniques Cont. . . . . . . . . . . . 97 


\section{Chapter 1}

\section{Introduction}

The world's oceans and freshwater ecosystems are a sink for pollution, contamination, and excess greenhouse gases [1,2]. The health and natural cycling of aqueous environments are vital to maintaining healthy ecosystems and human civilization as we know it $[3,4]$. While scientists are working to better understand the mechanisms and impacts of pollution and climate change on our environment, engineers are tasked with equipping them with the instrumentation they need. This thesis is concerned with the engineering challenges prompted by the scientific need for environmental data to better study and understand climate change, carbon cycling and pollution in our oceans. Specifically, this thesis seeks to push the field of ocean and aqueous sensing from requiring large equipment or tedious sample collection followed by analysis in the lab, and move it toward miniaturized, accessible, and field-deployable techniques.

This thesis addresses the need to monitor dissolved carbon dioxide $\left(\mathrm{CO}_{2}\right)$ and methane $\left(\mathrm{CH}_{4}\right)$ as well as the emerging need to monitor microplastic contamination in aqueous environments. First, this introduction offers important background on the field of in situ and field deployable aqueous sensing, the significance of dissolved $\mathrm{CO}_{2}$ and $\mathrm{CH}_{4}$ in the environment, and scientific and technical background on gas sensing and gas-water equilibration. Following this, Chapter 2 presents a low-cost, portable solution for dissolved $\mathrm{CO}_{2}$ monitoring. Chapter 3 presents the design and simulation

results of novel nanophotonic solutions for dissolved $\mathrm{CH}_{4}$ sensing. Chapter 4 opens up to the emerging field of microplastic studies and monitoring and presents an in-depth 
literature review and perspective to motivate the development of field-deployable microplastic sensing techniques. This thesis concludes with a brief discussion of the future work in the field of portable, in situ ocean and freshwater sensing.

\subsection{Background on Greenhouse Gases and Aqueous Environments}

From 1960 to 2021, the atmospheric concentration of $\mathrm{CO}_{2}$ has risen approximately 100 ppm, to a current level of roughly 417 ppm [5]. Similarly, from 1985 to 2021, atmospheric levels of $\mathrm{CH}_{4}$ have risen $0.26 \mathrm{ppm}$ to a current level of $1.89 \mathrm{ppm}$ [5]. $\mathrm{CO}_{2}$ and $\mathrm{CH}_{4}$ are the two most important greenhouse gases [6,7], and their overabundance in our atmosphere has produced a global warming effect. Consequently, the increased global temperatures and concentrations of $\mathrm{CO}_{2}$ have interfered with the chemistry of ocean and freshwater aqueous environments [8]. This has implications in ocean acidification [2] and how carbon is cycled through aqueous environments [9]. This thesis is interested with how to enable scientists to better study the relationships our oceans and freshwater ecosystems have with the carbon cycle and changing climate.

\subsubsection{Measuring the carbon cycle in aqueous environments}

There are multiple metrics you can interrogate to evaluate the carbon content and export of water ecosystems. These metrics include dissolved organic carbon (DOC), dissolved inorganic carbon (DIC), stable and radio-carbon isotope composition $\left(\delta^{13} \mathrm{C}\right.$ and $\left.{ }^{14} \mathrm{C}\right)$, dissolved $\mathrm{CO}_{2}\left(\mathrm{pCO}_{2}\right)$, dissolved $\mathrm{CH}_{4}$, and flux of $\mathrm{CO}_{2}$ and $\mathrm{CH}_{4}$.

Dissolved organic carbon (DOC) encompasses the entirety of organic matter contained in a sample of water [10], including $\mathrm{CH}_{4}$, natural materials, and man-made pollutants such as polychlorinated biphenyls (PCBs) [11,12]. Dissolved inorganic carbon (DIC) includes $\mathrm{CO}_{2}$, bicarbonate $\left(\mathrm{HCO}_{3}{ }^{-}\right)$, and carbonate $\left(\mathrm{CO}_{3}{ }^{-}\right)$[13]. Table 1.1 lists theses metrics alongside the important information they provide scientists as well as how they are traditionally measured. 


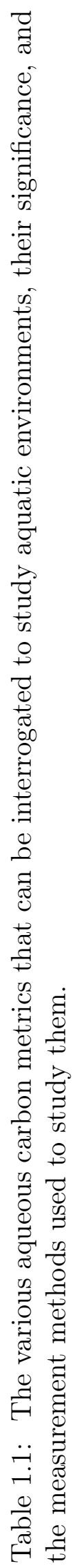


Of the metrics in Table 1.1, investigations of dissolved $\mathrm{CO}_{2}$ and $\mathrm{CH}_{4}$ are motivated by recent findings that ecosystems like estuaries, coastal and inland wetlands, and lakes store and emit these gases [26-31]. Ecosystems like these are emerging as key players in the global carbon cycle budget. Further data collection has much to reveal about the mechanisms of carbon cycling through these aqueous ecosystems [26]. Instruments that can enable rich temporal and spatial dissolved $\mathrm{CO}_{2}$ and $\mathrm{CH}_{4}$ datasets will drive this effort. Sections 2.1 and 3.1 provide a richer look at the motivations of dissolved $\mathrm{CO}_{2}$ and $\mathrm{CH}_{4}$ studies.

\subsection{Background on In Situ and Field-Deployable Aque- ous Sensing Technology}

The state-of-the-art in dissolved gas sensing is reflected in the methods and instrumentation currently used by ocean science research groups and institutions. Perhaps the most familiar sensing equipment to ocean researchers are conductivity, temperature, depth measurement instruments (CTDs) incorporated among a rosette of Niskin bottles [32]. Figure 1-1 shows a CTD rosette where the Niskin bottles are visible in a circle around the CTD which is housed in the middle. The conductivity measurement combined with the temperature and pressure measurements enable the calculation of water salinity $[32,33]$.

The Niskin bottles capture water samples at different depths of the water column while the CTD makes continuous measurements, returning a vertical profile of the water contents at the ship's location. The bottle contents are immediately subsam-

pled into collection vials and a chemical preservative is often added to them [34]. The samples are later analyzed in a lab, either in a ship lab [35] or after the cruise has concluded and the samples are returned to the main research campus. Analysis in the lab enables a researcher to analyze the sample with the entire suite of instruments available in their laboratory. Similar bottle sampling methods are also used for freshwater sampling, collected by hand or with an automated sampler [36] and returned 


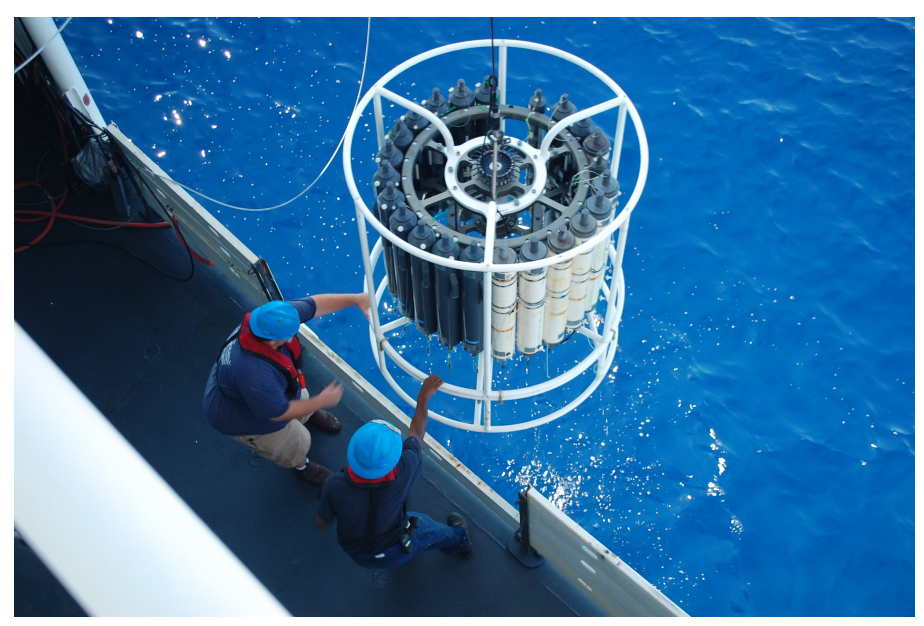

Figure 1-1: A CTD rosette being recovered on a National Oceanic and Atmospheric Administration (NOAA) ship. Credit: Courtesy of Officers and Crew of NOAA Ship PISCES; Collection of Commander Jeremy Adams, NOAA Corps, licensed under CC BY 2.0

to lab for analysis. This bottle-collection approach is the convention in the field of dissolved gas analysis, dating back to the 1970s [37] and is still used in practice today. For example, a 12-year study by Laach et al. (2016) was conducted to evaluate the seasonal variability of carbon export from peatlands and the $\mathrm{CO}_{2}$ and $\mathrm{CH}_{4}$ measurements were made using sample bottle collection followed by lab analysis with a gas chromatograph and a flame ionization detector (GC-FID) [38]. The drawbacks of these sampling techniques for dissolved gas analysis is that they are inherently time consuming, as sample collection and analysis happen at different locations and times. These tedious methods lead to temporally and spatially sparse data sets.

\subsubsection{Gas Extraction and Equilibration}

This section introduces the basic chemistry of gas equilibration in air and water and the state-of-the-art in gas extraction methods.

\section{The Chemistry of Dissolved Gas}

When gas is in contact with water, Henry's Law states that the gas will dissolve into the water proportionally to the partial pressure of the gas until the dissolved 


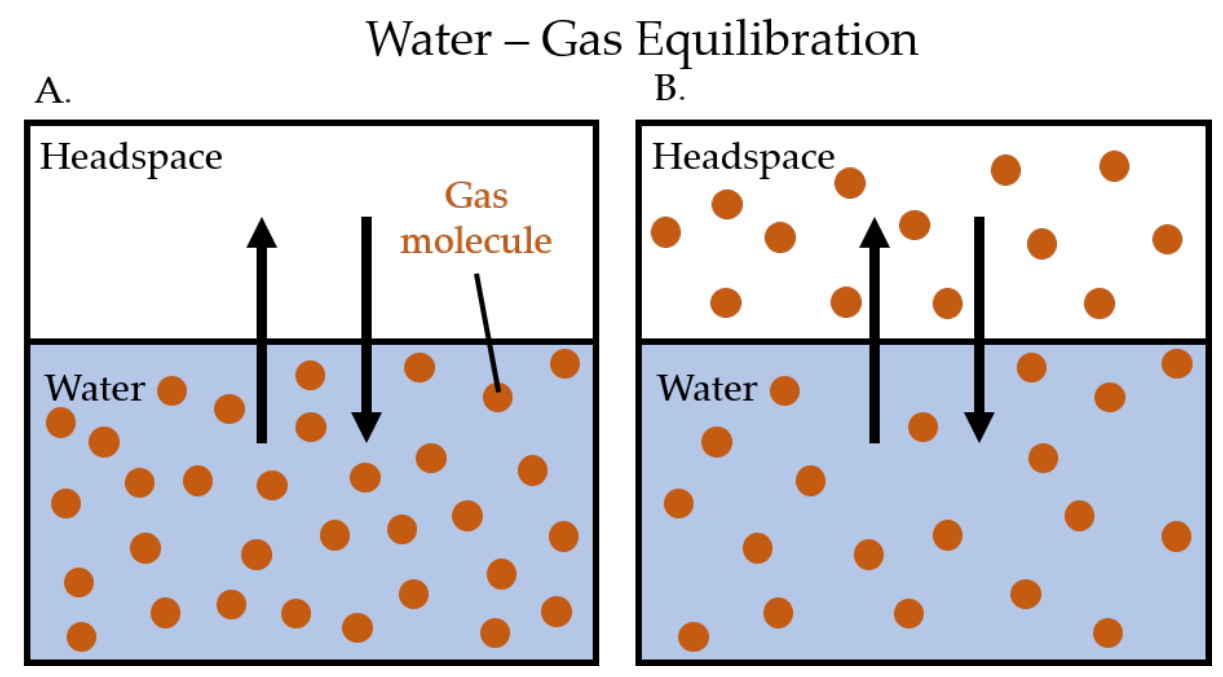

Figure 1-2: An illustration of headspace equilibration. (A) A water sample containing the target gas (orange circles) is placed in a container with a headspace of known target gas concentration (in this image, none). (B) Once the headspace and water equilibrate, the headspace gas concentration can be measured and used to calculate the original dissolved gas concentration.

and undissolved gas reach equilibrium [39]. Each gas has a Henry's law volatility constant, $K_{H}$, defined experimentally for certain temperatures:

$$
K_{H}=\frac{p_{a}}{c_{w}}
$$

where $p_{a}$ is the partial pressure of the gas in the air and $c_{w}$ is the concentration of gas in the water [39]. Henry's law is key to gas equilibration and extraction. Different techniques take advantage of Henry's law and varied methods of gas transfer to equilibrate dissolved gas from the sample water into the headspace. For any extraction system, the measured final concentration of the target gas in the headspace can be used to calculate the true dissolved gas concentration.

In the following sections, I present the principles of the most common state-ofthe-art gas extraction and equilibration techniques. 


\section{Headspace Equilibration}

Headspace equilibration is the most traditional and simple method of gas extraction [40]. In headspace equilibration, a water sample is collected in a gas-tight vial and the headspace in the vial above the water is filled with a gas of known target gas concentration [34]. In many cases a gas free of any of the target gas is used in the headspace, such as helium gas for dissolved $\mathrm{CO}_{2}$ or $\mathrm{CH}_{4}$ analysis [34]. The vial is then shaken vigorously, allowing the headspace and water to come to equilibrium via Henry's law. The headspace is then injected into a gas sensor, such as infrared absorption spectrometer like the Ultraportable Greenhouse Gas Analyzer (ABB-Los Gatos Research, Inc., Mountain View, CA, USA) or a gas chromatograph and the dissolved gas concentration can be calculated. The key equations for calculating the original dissolved gas concentration of a sample of water are as follows. Assume that a water sample of volume $V_{W}$ containing $\mathrm{CO}_{2}$ as its target gas is placed in a vial with a headspace of volume $V_{H S}$ and the headspace is filled with helium gas (like the situation illustrated in Figure 1-2). The desired measurement is the dissolved $\mathrm{CO}_{2}$ concentration in the water, $\left[\mathrm{CO}_{2}\right]$ :

$$
\left[\mathrm{CO}_{2}\right]=\frac{n_{\mathrm{CO}_{2}}}{V_{W}}
$$

where $n_{\mathrm{CO}_{2}}$ is the molar quantity of $\mathrm{CO}_{2}$ in the entire vial. Once equilibration takes places between the water and the headspace the molar quantity is

$$
n_{\mathrm{CO}_{2}}=n_{\mathrm{CO}_{2}, \mathrm{HS}}+n_{\mathrm{CO}_{2}, W}
$$

The gas analyzer measures the partial pressure concentration of the equilibrated headspace, $p_{\mathrm{CO}_{2}, \mathrm{HS}}$, in parts per million (ppm). From this, $\left[\mathrm{CO}_{2}\right]$ is calculated via:

$$
\left[\mathrm{CO}_{2}\right]=\beta \frac{p_{\mathrm{CO}_{2}, \mathrm{HS}} \cdot P_{\mathrm{HS}}}{R(2.73 .15+T)} \quad[\text { Magenet al. } .(2014)]
$$

where $\beta$ is gas specific Bunsen coefficient, $\mathrm{R}$ is the universal gas constant, $\mathrm{T}$ is the sample temperature, and $P_{H S}$ is the pressure of the headspace. Headspace 
equilibration techniques are relatively simple to implement and are useful when the sample needs to be stored or transported prior to analysis. However, storage for extended periods of time can make the measurement vulnerable to contamination from leaking or pulling air into the vial from the atmosphere [34].

\section{Membrane}

Perhaps the next most intuitive method of gas extraction for dissolved gas analysis is the use of a gas-permeable membrane. In this technique, a headspace is housed behind a thin-film gas-permeable membrane, through which gas from the water equilibrates. The gas concentration of the equilibrated headspace is measured and used to calculate the dissolved gas concentration in the water. Membrane equlibration is suitable for in situ dissolved gas measurements, as the membrane can passively equilibrate with the dynamic environment over long time scales. Membrane techniques have been used for in situ sampling of dissolved gases since as early as the 1970s [41,42]. Since then, membrane technology has been well studied and optimized techniques are commercially available, such as $3 M^{T M}$ Liqui-Cel ${ }^{T M}$ Membrane Contractors, which work at a variety of flow speeds and efficiencies. These technologies can achieve relatively fast equilibration times for $\mathrm{CO}_{2}$ [43]. Additionally, ABB-Los Gatos Research sells a dissolved gas extraction unit which uses a membrane contractor in a flow through regime [44].

\section{Spray}

In spray-type gas equilibrators, a showerhead stream of sample water is sprayed into the headspace [43]. The transport of the water droplets through the headspace encourages gas transfer and speeds the process of equilibration. Included within this is the Weiss-type [45] equilibrator. The spraying of the showerhead is similar to the bottle-shaking step in the simple bottle collection and headspace equilibration tech-

nique described above. A spray-type gas equilibrator is used in this thesis to create water samples with known dissolved gas concentration in Chapter 2. 


\section{Bubbling}

Gas equilibration techniques can use bubbling as a means to increase and speed up gas transfer from the water to the headspace [46]. In this situation, gas is bubbled through the sample water in either an open or closed loop. In closed loops, gas is fed back through the water to encourage more diffusion of the headspace gas with that of the water. Certain parameters of the feed-back bubbling increase mass flow between the gas within the bubbles and the surrounding water [47].

Key factors to consider include bubble size and bubble speed. As the size of the individual bubbles increases, the interphase mass transfer coefficient between the water and gas decreases [47]. This means that smaller bubbles are better for gas equilibration applications. As bubble velocity increases, the interphase mass transfer coefficient between the water and gas is relatively constant, but high velocities perform better [47].

\section{Marble}

In marble-type gas equilibration, sample water is flowed downward over a cylinder of marbles while a close loop of air circulates from the headspace up through the marbles [48]. Equilibration occurs in this case because of turbulent flow in the system.

Kortzinger et al. (2009) and Webb et al. (2016) present comprehensive reviews of membrane-, showerhead- (and Weiss-) bubble- and marble-type air-water equilibrators for dissolved $\mathrm{CH}_{4}$ and $\mathrm{CO}_{2}$ analysis [43,46], demonstrating response times as low as 34 seconds for $\mathrm{CO}_{2}$ with membrane type calibrators.

\subsubsection{Gas Analysis}

Following gas extraction, gas analysis can be performed with a range of sensor types. Here the gas analyzers used in current state-of-the-art field-portable measurement system are highlighted.

Infrared spectroscopy and mass spectroscopy are two techniques currently used

to measure dissolved gas in situ in the ocean and coastal environments [49-52]. For 
these techniques, the gas concentration is measured in the gas phase, requiring that the instruments be coupled to a gas extraction system [49]. Gas extraction and equilibration can have time responses on the scale of hours and very limited volumes of gas can be extracted, impacting overall time response and limits of detection of the sensor [18]. A commercial instrument designed for the analysis of extracted gas is the aforementioned ABB-Los Gatos Ultraportable Greenhouse Gas Analyzer, a cavity enhanced infrared absorption spectrometer which weighs $15 \mathrm{~kg}$ and is roughly the size of a briefcase [53]. Other infrared absorption spectroscopic instruments have been deployed on underwater vehicles to monitor subsea dissolved gas, employing complex fluid and gas exchange systems and laser diode sources [51,54]. ProOceanus offers commercially available dissolved $\mathrm{CO}_{2}$ and $\mathrm{CH}_{4}$ sensors based on non-dispersive infrared (NDIR) sensing chambers coupled to a gas permeable membrane. This instrument weighs only $0.53 \mathrm{~kg}$ and measures $11 \mathrm{~cm}$ in length, but is relatively high cost [55]. The CONTROS HydroC $\mathrm{CH}_{4}$ sensor also couples absorption spectroscopy and a gas permeable membrane, but is roughly $50 \mathrm{~cm}$ in length and $12.5 \mathrm{~kg}$ in weight, and also has high cost [56]. Similar to absorption spectroscopy measurements, in situ mass spectrometers can be used to monitor environmental dissolved gas at low concentrations, but are large, high cost, and power intensive [49].

The large size and mass of these instruments greatly limits the range of locations where measurements can be made and the platforms that can use them. The development of small, portable dissolved gas sensors, which are either handheld or deployable on underwater vehicles, would enable more widespread studies and measurements in waterways that are currently routinely inaccessible. Additionally, developments of such instruments will provide labs the means to purchase or make their own low-cost systems instead of needing to purchase extremely costly ones. In the following Chapter, a gas extraction and analysis system is presented which seeks to move dissolved gas sensing from an expensive, cumbersome endeavor to a low-cost and field-portable method, which balances the trades of cost, size, measurement accuracy, and robustness. 


\section{Chapter 2}

\section{A Low-Cost Gas Extraction and}

\section{Measurement System (LC-GEMS) for dissolved gas analysis in aqueous}

\section{environments}

\section{$2.1 \quad$ Introduction}

Estuaries, lakes, coastal salt marshes, and fresh inland wetlands have been found to store and emit carbon dioxide $\left(\mathrm{CO}_{2}\right)$ and methane $\left(\mathrm{CH}_{4}\right)$ and are recognized as emerging players in the global carbon cycle budget [26-31]. The majority of lakes globally are supersaturated with $\mathrm{CO}_{2}$ [57]. In a recent study in the Alpine area of the Eastern Alps, Pighini et al. (2018) found that 39 of 40 investigated lakes were supersaturated with both $\mathrm{CO}_{2}$ and $\mathrm{CH}_{4}$ [58]. The near-surface dissolved concentrations of $\mathrm{CO}_{2}$ and $\mathrm{CH}_{4}$ were found to exceed atmospheric levels by factors of approximately 2 and 400 times respectively [58]. Additionally, in coastal salt marshes and estuaries, significant flux of $\mathrm{CO}_{2}$ and $\mathrm{CH}_{4}$ has been observed [28]. The flux of $\mathrm{CO}_{2}$ and $\mathrm{CH}_{4}$ is these diverse in-land and coastal environments has significant temporal and spatial variability due to factors such as salinity, sedimentation, microbial activity, and tide [29]. In light 
of findings like these, it has been recognized by the scientific community that more measurements in ecosystems like reservoirs, estuaries, rivers, and brackish marshes are necessary to generate data for building global $\mathrm{CO}_{2}$ and $\mathrm{CH}_{4}$ emission models [59].

This thesis chapter will address the need for accessible, portable, and low-cost methods to monitor $\mathrm{pCO}_{2}$ through the design of a toolbox-sized gas extraction and measurement system and subsequent field testing and validation in a salt marsh.

\section{$2.2 \quad \mathrm{pCO}_{2}$ in salt marshes}

Salt marshes are suitable ecosystems to evaluate a $\mathrm{pCO}_{2}$ sensor because they capture and store carbon in their sediment and thus play a role in both emitting and absorbing $\mathrm{CO}_{2}$ to and from the atmosphere [60]. The controls and balances of the $\mathrm{CO}_{2}$ cycling in salt marsh ecosystems are not well understood [61]. The $\mathrm{CO}_{2}$ exchange in marsh ecosystems can vary significantly on very small spatial scales (meters) due to the varied distribution of plant life and the presence of still pools [62]. These factors make salt marshes interesting locations to study the daily fluctuations of $\mathrm{pCO}_{2}$ concentration as a field validation for a field-portable dissolved gas measurement system.

$\mathrm{CO}_{2}$ flux from salt marshes varies throughout the course of the day due to the daily photosynthesis cycle of plant life. During the day, $\mathrm{CO}_{2}$ is consumed by the plant life, lowering the concentration of $\mathrm{CO}_{2}$. At night, the plants respire their $\mathrm{CO}_{2}$ stores, increasing $\mathrm{CO}_{2}$ concentration. Additionally, the tidal cycle can influence the $\mathrm{CO}_{2}$ concentration in a salt marsh: daytime high tides reduce the $\mathrm{CO}_{2}$ uptake into water [63]. An observational study of the diurnal (daytime and nighttime) fluctuations of $\mathrm{pCO}_{2}$ concentrations in a salt marsh should provide a dynamic dataset to investigate a $\mathrm{pCO}_{2}$ sensor.

\subsubsection{Measuring dissolved $\mathrm{CO}_{2}$ in aqueous environments $\left(\mathrm{pCO}_{2}\right)$}

Monitoring dissolved $\mathrm{CO}_{2}$ in surface waters is a means to study and quantify the $\mathrm{CO}_{2}$ production and flux from a water body [64]. For this reason, many groups have investigated means to measure the dissolved $\mathrm{CO}_{2}$ content in surface waters across 
lakes, rivers, and coastal ecosystems [50,65].

Atmospheric $\mathrm{CO}_{2}$ sensors measure $\mathrm{CO}_{2}$ concentration in the gas phase in parts per million (ppm) by volume. Dissolved $\mathrm{CO}_{2}$ is often represented as the partial pressure of $\mathrm{CO}_{2}\left(\mathrm{pCO}_{2}\right) \cdot \mathrm{pCO}_{2}$ relates to the parts per million by volume of $\mathrm{CO}_{2}$ via

$$
p C O_{2, \mu a t m}=C O_{2, p p m} \cdot 1 \times 10^{-6} \frac{1}{p p m} \cdot T D G P
$$

where TDGP is the total dissolved gas pressure of the water sample [66].

The sensors used in this thesis chapter measure $\mathrm{CO}_{2}$ in ppm. Thus, ppm is the unit of measurement that will be used in the calibration experiments of the system presented here and the term dissolved $\mathrm{CO}_{2}$ will be used rather than $\mathrm{pCO}_{2}$ when the TDGP is unknown. For the field work in this thesis chapter, surface waters will be the target, meaning the water depth of analysis will be $30 \mathrm{~cm}$ at maximum. For near-surface measurements like this, TDGP can be assumed to be atmospheric [67], allowing the determination of $\mathrm{pCO}_{2}$ via Equation 2.1 in these situations. As $P=1$ atm in this situation, the conversion is simple, but in future studies or uses of the LC-GEMS, accounting for the true TDGP will be an important consideration if the LC-GEMS is adapted for use at depth.

\subsubsection{State of the art in dissolved $\mathrm{CO}_{2}$ sampling and analysis}

Specific engineering efforts have been undertaken to measure dissolved gas content in freshwater lakes and rivers. Crawford et al. (2015) presented a high speed gas equilibrator which could profile the $\mathrm{pCO}_{2}$ content of lake surface waters when attached to the back of a boat, making use of the commercial ABB-Los Gatos Ultraportable GGA [65]. In another recent study, Nicholson et al. (2018) integrated the ABBLos Gatos Ultraportable GGA with a commercial gas extraction unit (Dissolved Gas Extraction Unit, ABB-Los Gatos Research, Inc.), which uses a membrane contactor, to profile the dissolved $\mathrm{CH}_{4}$ and $\mathrm{CO}_{2}$ content of a river surface [50]. Xiao et al. (2020) present a hybrid, low-cost gas extraction with an exceptionally fast time response $\left(t_{95 \%}=10\right.$ seconds for $\left.\mathrm{CO}_{2}\right)$ when integrated with commercial gas analyzers [68]. 
These emergent techniques show promise in making continuous, in situ gas extraction for gas sensors more accessible and portable. However, the techniques used by Nicholson et al. (2018), Crawford et al. (2015), and Xiao et al. (2020) all make use of large, expensive commercial gas analyzers (Los Gatos GGA, GasScouter G4301, and Picarro G2132-i) which each cost upwards of tens of thousands of dollars. The extraction system developed by Crawford et al. (2015) was integrated onto a small research boat which supported all the intake pumps and sensing equipment [65]. The Dissolved Gas Extraction Unit used by Nicholson et al. (2018) is large and are difficult to transport to the sample site by hand. Thus, despite the existence of these instruments and advances making them more rapid and portable, many lab and community groups still rely on bottle-sample collection methods because the extraction and portable analysis equipment is prohibitively expensive and also cumbersome and complicated to transport and deploy.

For this reason, methods are needed that bridge the gap between affordability and continuous, in situ dissolved gas monitoring. This thesis presents a sensing system that meets this need by balancing the trade-offs of data quality, portability, price, and robustness through the implementation of a low-cost gas extraction system and low-cost commercial off-the-self $\mathrm{CO}_{2}$ sensors.

\subsection{Design of the Low-Cost Gas Extraction and Mea- surement System (LC-GEMS)}

In the approach presented here, a low-cost gas extraction and measurement system (LC-GEMS) is built from three interconnected systems to create a portable dissolved gas sensor for shallow water applications: 1) a gas extractor, 2) a gas sensor, and 3) a power routing stage (Figure 2-1). This section presents the design of each of these components. The gas extractor design is based on the FaRAGE (Fast-Response Automated Gas Equilibrator) system presented in Xiao et al. (2020) [68], redesigned and built here using low-cost equipment, totaling just under $<\$ 350$ cost (Table 


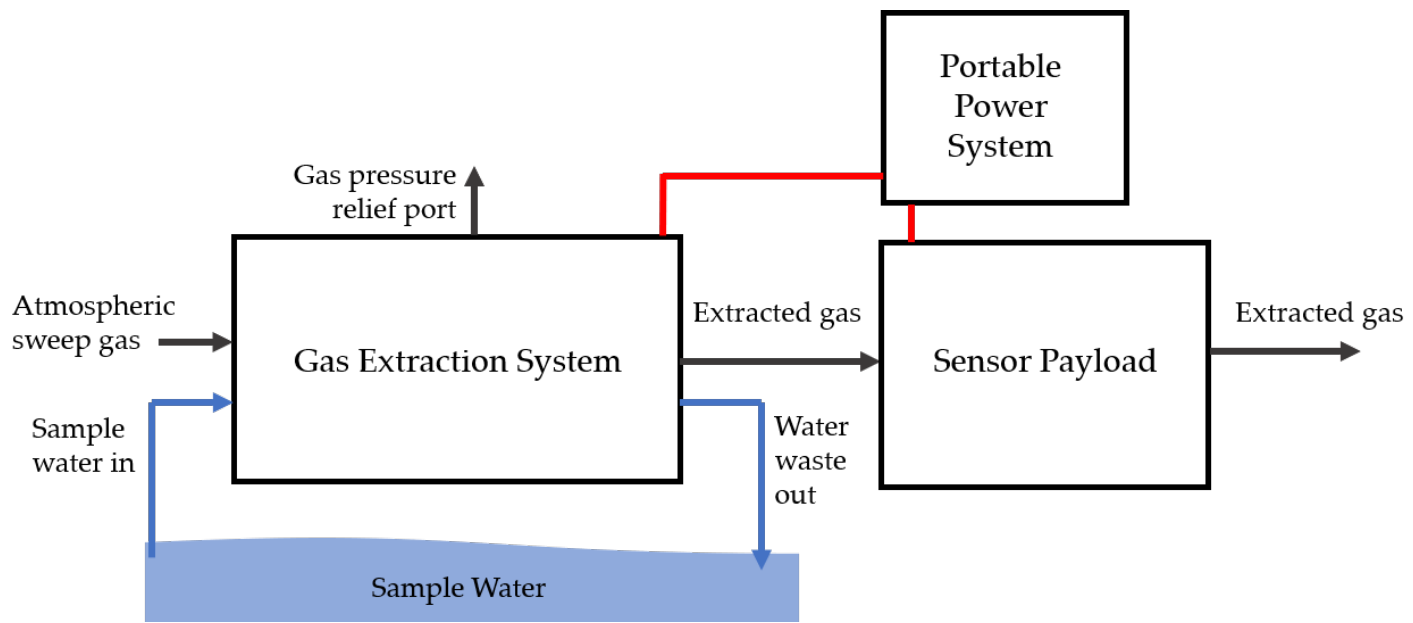

Figure 2-1: System diagram of the LC-GEMS. The gas extraction system takes in the input sample water as well as atmospheric air as sweep gas. The extracted gas sample is fed to the sensor payload and the waste water is output back to the environment. Both the gas extraction system and sensor payload are powered by a portable power system. The gas paths are drawn in black, the water paths are drawn in blue, and the power paths are drawn in red.

2.2), and remodeled for handheld use in the field. The gas analysis stage is built from a low-cost microcontroller, datalogger, commercial of-the-shelf (COTS) pressure, humidity, and temperature sensors (Adafruit BME280), and a COTS $\mathrm{CO}_{2}$ gas sensor (CO2meter.com K30) which collectively cost $\$ 213$ (Table 2.2). The portable power routing system relies on a waterproof electronics box which distributes power via press buttons and can distribute power from any $12 \mathrm{~V}$ battery. At a total cost of just under $\$ 600$ and with a simple to replicate design, this system enables the in situ investigation of dissolved gas concentration trends at slightly lower resolution than expensive state of the art equipment.

\subsubsection{Gas Extraction System}

The FaRAGE system presented in Xiao et al. (2020) has a $95 \%$ time response $\left(t_{95 \%}\right)$ of 10 seconds and nanomolar range sensitivity, when coupled to state of the art gas analysis equipment (GasScouter, Picarro, Inc, Santa Clara, CA, USA). This time response is among the fastest of current gas extraction techniques [43]. The design 
incorporates bubble and Weiss type equilibrator techniques into a flow-through system that has a headspace of minimal volume. The FaRAGE system works as follows (summarized from Xiao et al. (2020)): The sample water and a sweep gas are injected into a gas-water mixing unit and then fed through two meters of tubing for further mixing. The mixed sample water and sweep gas are then sprayed downward into a gas-water separation unit, with the water being removed and the headspace being fed to the gas analyzer stage (through desiccant and filters to ensure the analyzers are not damaged by water). The speed of the FaRAGE technique is a result of the minimal headspace and hybrid flow-through design. Xiao et al. (2020) provide supplemental information detailing the chemical and physical theoretical background of the design [69].

Xiao et al. (2020) built their gas-water mixing and gas-water separation units from syringes and present the design in their supplement [69]. The use of plastic syringes for stages makes the FaRAGE system low-cost and accessible. However, the extraction system uses expensive mass flow controllers, pumps, and meters to regulate the flow of their system, as well as a compressed gas cylinder for the sweep gas. The cost of these items (over \$2000) and the great cost of the gas analyzers used at the analysis stage (tens of thousands of dollars) make this system still prohibitively expensive for some groups, such as research groups at academic institutions. Additionally, the weight of all this equipment (over $15 \mathrm{~kg}$ ) is not easily transportable by hand.

The LC-GEMS adapts the gas-water mixing and separation stages of the FaRAGE system into a lost-cost gas extraction system (all parts and costs listed in Table 2.2). The key adaptions and differences between the FaRAGE system and the gas extraction system in the LC-GEMS are detailed in Table 2.1. The gas-water mixing and separation stages are built following the FaRAGE technique, using plastic syringes as the stage housings [69]. Rather than use mass flow controllers, which would cost over $\$ 2000$, the water and sweep gas flows are controlled by voltage regulated COTS peristaltic and vacuum pumps respectively in the LC-GEMS. The rate of the water inlet and outlet flow is controlled by regulating the supply voltage to the peristaltic pumps with $12 \mathrm{~V}$ Step-Up/Step-Down Voltage Regulators. This maintained a water flow of 


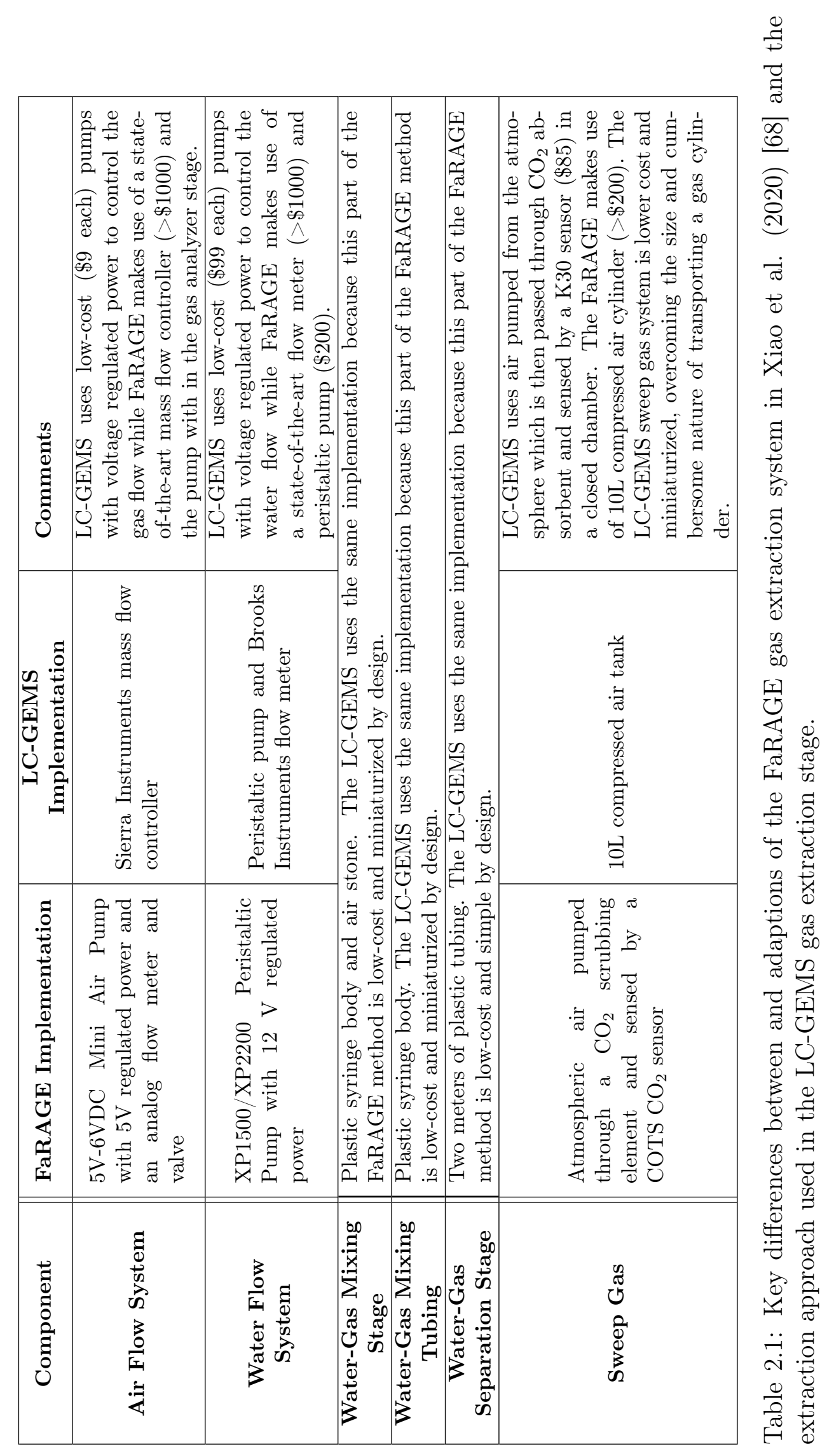




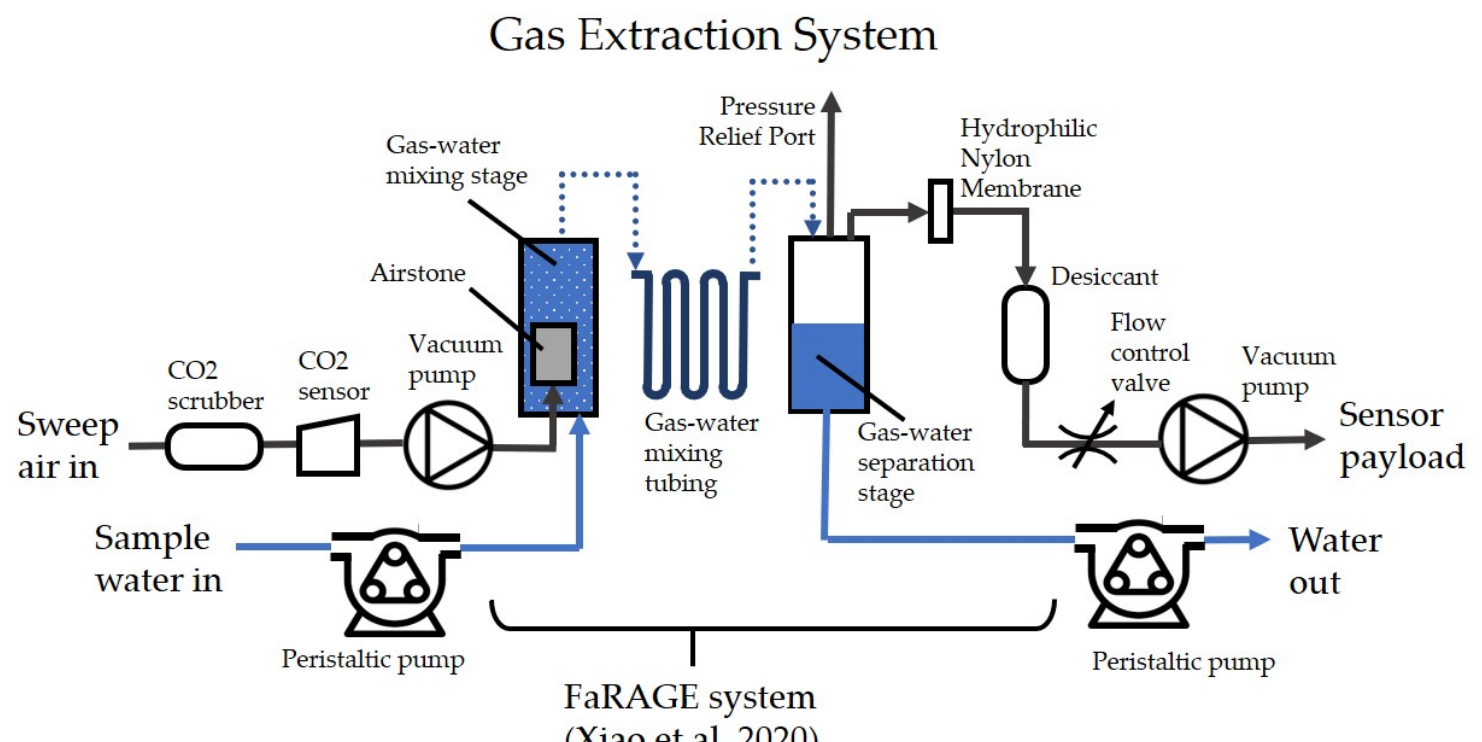

(Xiao et al. 2020)

Figure 2-2: The gas extraction system used in the LC-GEMS. The middle labeled portion (gas-water mixing state, gas-water mixing tubing, gas-water seperation stage, and the membrane and desiccant drying elements) are of the FaRAGE system as presented by Xiao et al. (2020) [68]

approximately $500 \mathrm{ml} / \mathrm{min}$ for the peristaltic pumps used. The rate of the sweep gas flow is controlled by supplying the vacuum pumps with $5 \mathrm{~V}, 2.5 \mathrm{~A}$ Step-Down Voltage Regulators. This maintains the inlet sweep gas at a flow of approximately $1.5 \mathrm{~L} / \mathrm{min}$. The outlet flow of the extracted gas is also regulated by a manual flow control valve on an analog flow meter (Figure 2-3), adjusting it to $1 \mathrm{~L} / \mathrm{min}$. The use of low-cost pumps without precise flow rate control comes at the cost of precision in the mixing ratio of gas and water. The composition of the gas reaching the sensor from the extraction stage depends on these flow rates. For this reason the LC-GEMS performance and extraction efficiency is best captured by a lab calibration across several known dissolved gas samples.

The passive pressure regulation in the water-air mixing chamber allows the air and water flow balance to be slightly imprecise without endangering the sensor payload. This ensures that the water-air mixing chamber does not over-pressurize and begin to pump water to the sensor payload. 
Another key difference between the FaRAGE system's extraction method and the method taken in the LC-GEMS is that, rather than using air from a gas canister, the sweep gas for the LC-GEMS is atmospheric air pumped through a syringe filled with Litholyme $\mathrm{CO}_{2}$ absorbent pellets (Allied Heathcare Products Inc., St. Louis, MO, USA), that absorb $\mathrm{CO}_{2}$ from a gas flow.

In total, the LC-GEMS extraction stage costs just under $\$ 350$ (Table 2.2), as opposed to a cost of over $\$ 4,000$ for the FaRAGE gas extraction system. Figure 2-2 outlines the components used in the LC-GEMS extraction system. The entire extraction system is held within a hand-portable $(36 \times 20 \times 20 \mathrm{~cm})$ plastic toolbox (Figure 2-3).

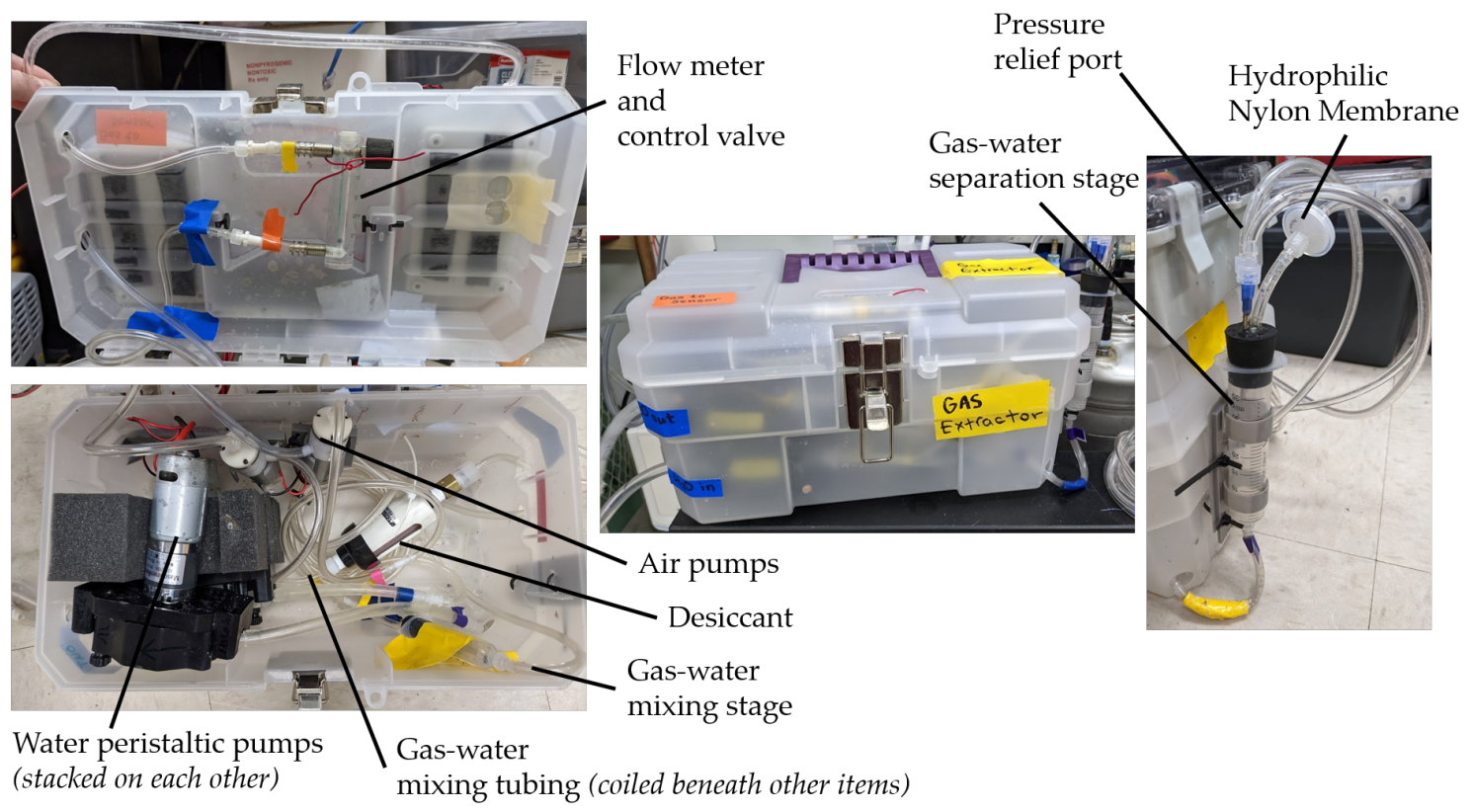

Figure 2-3: Photographs of the gas extraction system used in the LC-GEMS. The same syringe design as presented in Xiao et al. (2020) is used for the gas-water mixing and separation stages, while the water flow control, gas flow control, and sweep gas systems are adapted as explained in Section 2.1. The entire LC-GEMS extraction system is housed inside a plastic hand-portable toolbox which is lightweight and easy to transport. 


\subsubsection{Sensor Payload}

The sensor payload system contains three components: 1) the extracted dissolved gas analysis chamber, with $\mathrm{CO}_{2}$ sensor, and pressure $(\mathrm{P})$, humidity $(\mathrm{H})$, and temperature (T) sensors 2) the sweep gas sensing chamber, which houses an additional $\mathrm{CO}_{2}$ sensor and 3) the electronics for control, communication, and data logging. Figure 2-4 illustrates the extracted dissolved gas and sweep gas chambers as well as their supporting electronics.

\section{Sensor Payload System}

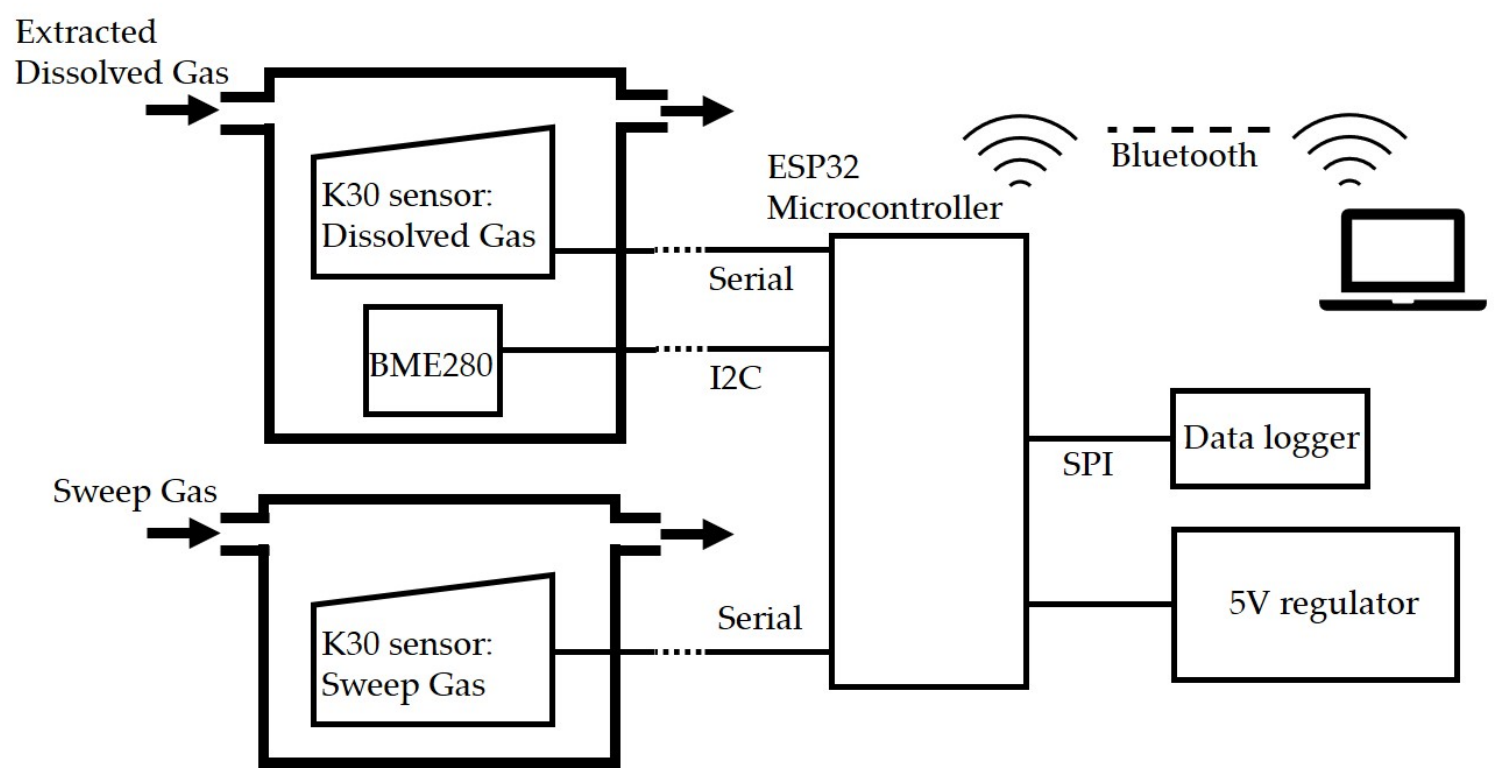

Figure 2-4: The sensor payload and electronic system of the LC-GEMS. $\mathrm{CO}_{2}$ gas sensors, housed in air tight chambers, are used to monitor both the inlet sweep gas to the LC-GEMS and the outlet gas from the LC-GEMS. The BME280 sensor monitors pressure, temperature, and humidity in the outlet gas chamber. An ESP32 microcontroller controls and communicates with the sensors and logs the data and also send the data via bluetooth to a computer or mobile device for live monitoring.

\section{$\mathrm{CO}_{2}$ sensor}

Infrared (IR) transmittance and absorption spectroscopy are a means to probe and measure the interaction of IR light with the internal bond structure of a gas [70]. The chemical bonds within a gas molecule absorb certain wavelengths of light at 
different magnitudes, depending on the nature of the chemical bond. For example, the symmetric $\mathrm{C}=\mathrm{O}$ stretch bond in $\mathrm{CO}_{2}$ exhibits absorption at $1337 \mathrm{~cm}^{-1}$ [71], equivalent to a $7479.43 \mathrm{~nm}$ wavelength, in the mid-infrared region. Many state of the art $\mathrm{CO}_{2}$ sensors work off the principal of IR absorption spectroscopy and use high precision opto-electronics to achieve high sensitivity.

Fourier transform infrared spectroscopy (FTIR) is another method of infrared spectroscopy that makes use of interferometry and the mathematical technique of Fourier transform. All infrared frequencies are measured simultaneously via an interferometer, resulting in a fast and highly-sensitive measurement of a large frequency spectrum [72]. The raw measurement must be processed by a Fourier transform to interpret the data as a frequency spectrum.

Turning to the needs of the LC-GEMS, there are many characteristic required of a $\mathrm{CO}_{2}$ sensor for dissolved gas analysis that go beyond the requirements for simple atmospheric monitoring. Dissolved gas sensors must be resistant and robust against humidity because gas extraction often results in elevated moisture content in the gas sample. Dissolved gas sensors must also have a low detection limit and high sensitivity around atmospheric concentration (roughly $400 \mathrm{ppm}$ for $\mathrm{CO}_{2}$ ) to ensure detection in the range expected for the target ecosystem, which begins at near atmospheric levels for dissolved $\mathrm{CO}_{2}$ analysis.

IR absorption spectroscopy is vulnerable to moisture content because water is absorbent across a broad range of wavelengths and can interfere with the reading at the band associated with $\mathrm{CO}_{2}$ [73]. This is overcome in some sensing techniques by using reference cells that simultaneously measure the water vapor content and can correct for it in the final measurement [74]. In other infrared absorption measurement techniques, cross-correlation correction methods are used [73]. Cross-correlation correction is a data processing technique in which known interference relationships between gas are used to calculate their respective concentrations in unknown mixtures [73]. The LC-GEMS is targeted for applications where portability and accessibility are more important than maximal precision. For this reason, state of the art spectrometers, which are on the order of tens of thousands of dollars, are inappropriate. 
In recent years, the availability of $\mathrm{COTS} \mathrm{CO}_{2}$ sensors has grown in the market of atmospheric measurement because of widespread availability to low-cost light sources, detectors, and new fabrication techniques [75-77]. COTS $\mathrm{CO}_{2}$ sensors work off many different sensing principles, including, most prominently, metal oxide semi-conductor (MOS) types [78] and NDIR types [75]. Semiconductor gas sensors are vulnerable to humidity and can inhibit sensor performance [79] and for this reason MOS sensors were not pursued for the LC-GEMS.

Like IR and FTIR spectrometers, NDIR sensing works off the principle of infrared light absorption. The sensor is made from an IR light source and detector on opposite sides of a sample chamber [74]. The IR source often projects a broadband of IR light and filters are used either at the source or detector side to select a wavelength where $\mathrm{CO}_{2}$ has a strong absorption band [74]. When $\mathrm{CO}_{2}$ is not present in the chamber, the intensity of the IR source hitting the detector is maximized. As more $\mathrm{CO}_{2}$ fills the chamber, the intensity reading at the detector is linearly decreased as a function of $\mathrm{CO}_{2}$ concentation. The technique is low-cost because of its simplicity: the term non-dispersive means that no prism or grating is used to separate the light, which would complicate the design.

NDIR sensors have been widely adopted across many disciplines of gas sensing for their robustness, reliability, and low power usage [75,80,81]. NDIR sensors have previously been installed behind gas-water membranes for in situ $\mathrm{pCO}_{2}$ analysis and have shown good stability and robustness [75,77,82-87]. For these reasons, an NDIR sensor was chosen for LC-GEMS. Of the commercially available NDIR $\mathrm{CO}_{2}$ sensors, the K30 10,000ppm $\mathrm{CO}_{2}$ Sensor from CO2meter.com (CO2Meter.com, Ormond Beach, FL, USA) has been well studied and categorized by research groups for atmospheric sensing $[75,77,86,87]$. The K30 demonstrates excellent sensitivity for its low cost. Previous works have found that the $\mathrm{K} 30 \mathrm{CO}_{2}$ sensor is sensitive around and below atmospheric conditions and can achieve a root mean square error as low as $1.9 \mathrm{ppm}$ once corrections for humidity and temperate and calibrations were performed and applied [77]. Thus, the K30 NDIR sensor fits the needs of dissolved gas sensing by being robust against humidity, highly sensitive, and having a lower detection limit 
than atmospheric conditions.

\section{Gas Sensing Chambers}

Two K30 sensors are used in the LC-GEMS. One is used for monitoring the input sweep gas and another monitors the outlet gas from the LC-GEMS. The sweep gas and outlet gas K30 sensors are contained in two separate air-tight chambers (Figure 25 A-B). The LC-GEMS reading, $x$, is defined as the difference between the $\mathrm{CO}_{2}$ concentration in the input sweep gas and that at the LC-GEMS output:

$$
x=\mathrm{CO}_{2, \text { Outlet }}-\mathrm{CO}_{2, \text { Sweep }}
$$

The difference of these two $\mathrm{CO}_{2}$ readings is the raw extracted gas measurement of the LC-GEMS.

The inlet of the sweep gas chamber is attached via a tube to the plastic syringe body filled with $\mathrm{CO}_{2}$ absorbent (Litholyme) (Figure 2-5A). The outlet of the sweep gas chamber is attached to the vacuum pump, which injects the sweep gas into the gas-water mixing stage (see Figure 2-2). The outlet gas sensing chamber is attached via tubing to the outlet of the gas extraction stage (Figure 2-5). Within the outlet gas sensing chamber is also an $\mathrm{H}, \mathrm{T}$, and $\mathrm{P}$ sensor (Adafruit BME280; Figure 2-5D). Section 2.4 explains how the K30 measurement is calibrated with the BME280 sensor to account for the fluctuating $\mathrm{H}, \mathrm{T}$, and $\mathrm{P}$ conditions within the chamber.

\section{Supporting electronics and housings}

The sensing system is controlled by a microcontroller chip (ESP32, Espressif Systems, Shanghai Co., Ltd) which is equipped with built-in WiFi and Bluetooth connectivity capabilities. The ESP32-DevKitC-32D (Mouser Electronics, Mansfield, Texas, USA) was the specific development board used. Bluetooth communication was used both in the lab and in the field to collect and monitor data live for the LC-GEMS. In addition, a microSD card reader module was used to log data for the sensor.

All electronics as well as the gas outlet chamber are housed inside a 150x100x70 
A.

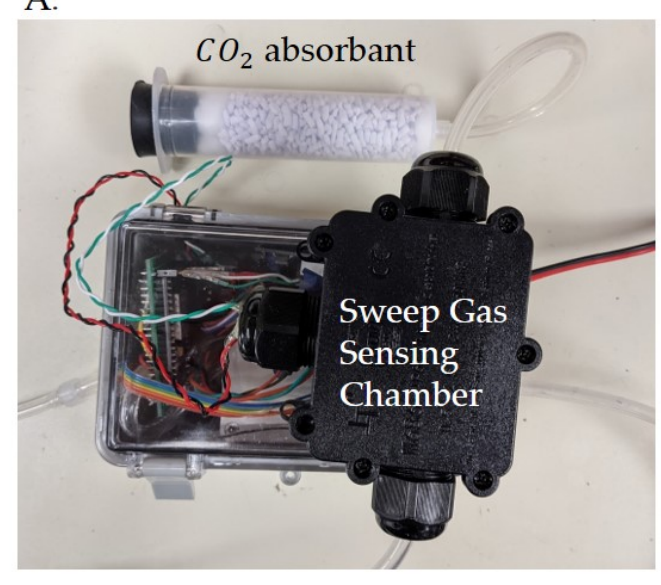

C.

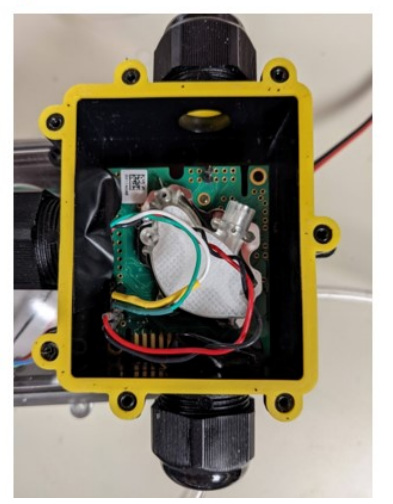

B.

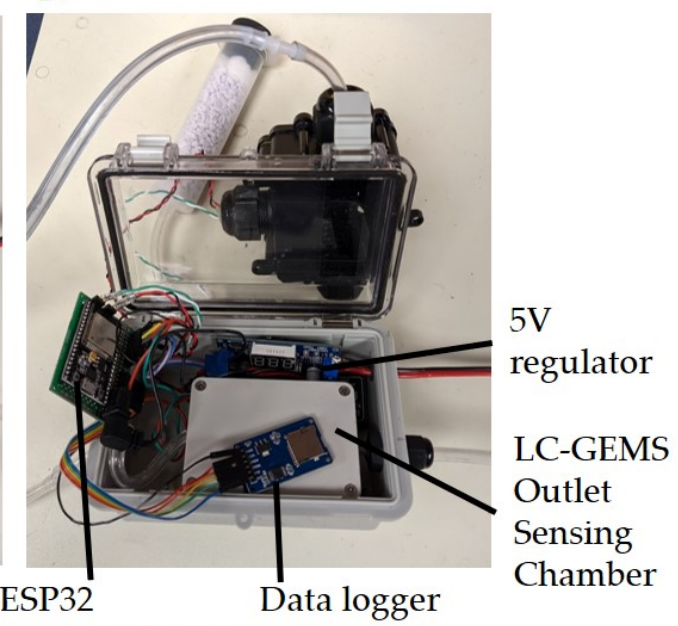

Microcontroller

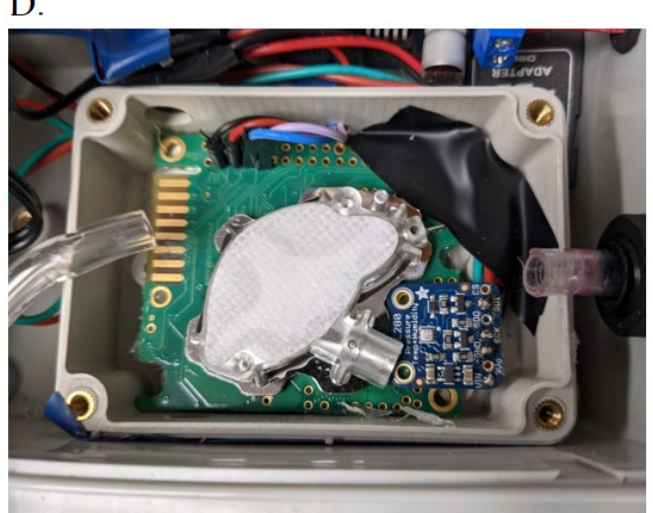

Figure 2-5: Photographs of the sensor payload system of the LC-GEMS. (A) The 150x100x70 mm waterproof electronics box holds the microcontroller, data logger, and $5 \mathrm{~V}$ regulator as well as the chamber for measuring the outlet gas from the LC-GEMS. The black enclosure sitting on top of this box is the sweep gas sensing chamber, which can be seen connected at its inlet to a syringe filled with $\mathrm{CO}_{2}$ absorbent. (B) The inside of the electronics box, showing the ESP32 microcontroller, data logger, $5 \mathrm{~V}$ regulator and the LC-GEMS outlet sensing chamber. (C) The inside of the sweep gas sensing chamber, containing the $\mathrm{K} 30 \mathrm{CO}_{2}$ sensor. (D) The inside of the LCGEMS outlet sensing, containing the $\mathrm{K} 30 \mathrm{CO}_{2}$ sensor and the BME280 humidity, temperature, and pressure sensor. 
mm waterproof electronics box (Figure 2-5B). The sweep gas chamber is housed in an $138 \times 105 \times 35 \mathrm{~mm}$ enclosure and is fastened to the top of the larger electronics box (the black enclosure in Figure 2-5A). The entire sensing payload system is attached to the top of the gas extraction enclosure (Figure 2-7).

\subsubsection{Portable Power System}

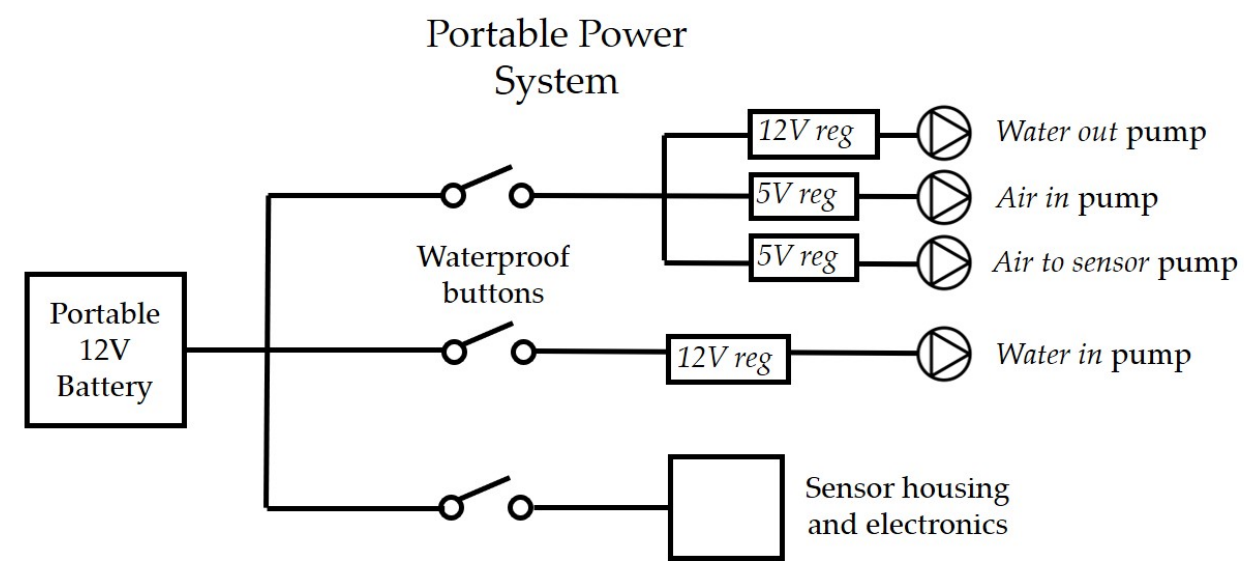

Figure 2-6: The power routing system used by the LC-GEMS. One outlet is attached to the portable $12 \mathrm{~V}$ battery pack to power the entire system. Waterproof buttons are used to power different components of the system.

Integral to the successful deployment of a sensor in the field is a portable power system that makes operating the system simple. As illustrated in Figure 2-6, the LCGEMS was designed to run off of one power outlet. The water outlet pump and both air pumps are turned on via button number one and the water inlet pump is turned on via button number two. This separation of the water inlet pump was to add an additional layer of safety to prevent flooding of the device, should a component fail. The sensors and their electronics were then turned on by button number three. The power routing system is attached to the top of the gas extraction housing, pictured in Figure 2-7. 

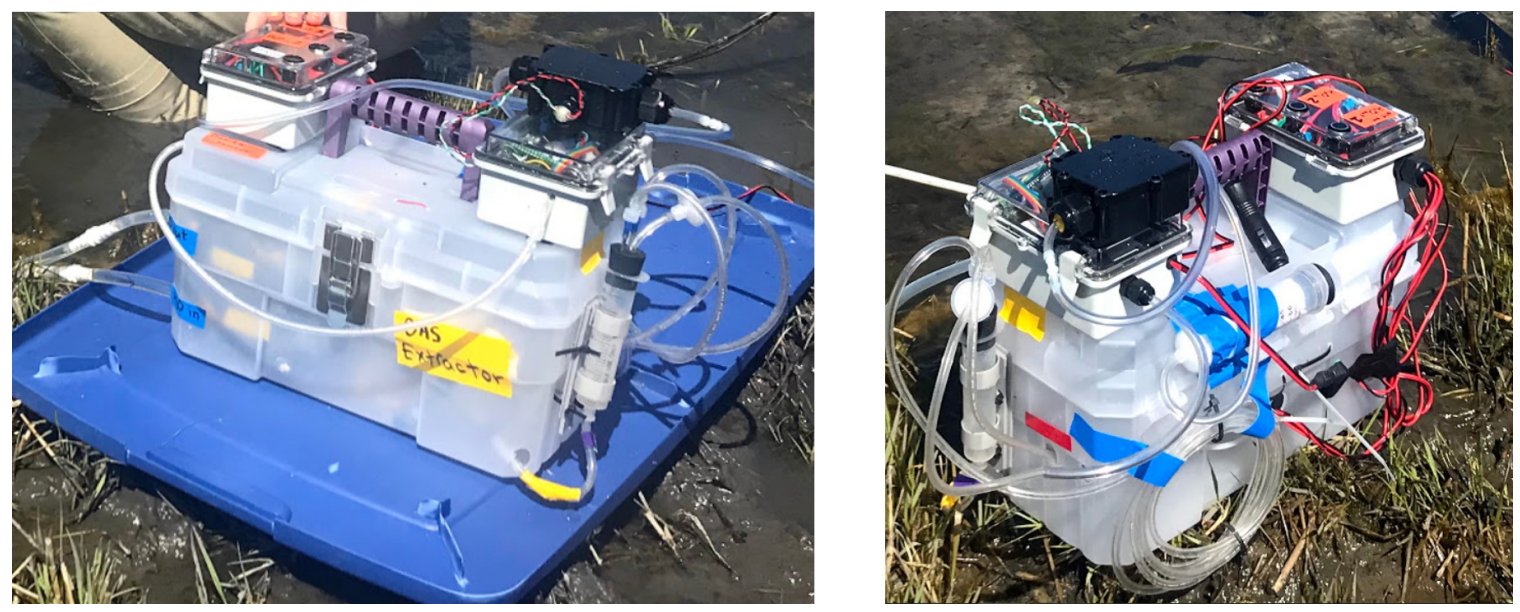

Figure 2-7: The full LC-GEMS system, front and back, in the field.

\subsubsection{Entire Integrated LC-GEMS}

The full LC-GEMS is picture in Figure 2-7. All of the components that are used in the LC-GEMS are presented in Table 2.2 alongside their cost.

\section{$2.4 \mathrm{~K} 30 \mathrm{CO}_{2}$ Sensor Calibrations}

Low cost COTS sensors, like the $\mathrm{K} 3 \mathrm{CO}_{2}$ sensors, do not match the precision and robustness of lab grade spectrometers and gas analyzers, like those used in other in situ dissolved gas measurement systems $[50,65,68]$. To correct for the relatively large (10s of ppm) root mean square errors (RMSEs) of these sensors, a multivariate linear regression model was used to correct for the effects of $\mathrm{H}, \mathrm{T}$, and $\mathrm{P}$ on the sensor reading, as previously presented for $\mathrm{K} 30 \mathrm{CO}_{2}$ sensors in Martin et al. (2017) [77]. Equation 2.4 presents the multivariate linear regression model used, in terms of $\mathrm{H}$, $\mathrm{T}$, and $\mathrm{P}$.

$$
C O_{2, p p m}=b_{0}+b_{H} \cdot H+b_{T} \cdot T+b_{P} \cdot P+e
$$

Where $b_{0}, b_{H}, b_{T}$, and $b_{P}$ are the regression coefficients and $e$ is the error coefficient. Prior to creating the multivariate linear regression model, the K30 sensors were calibrated according to their instruction manual [88], flowing nitrogen $\left(\mathrm{N}_{2}\right)$ gas into the 


\begin{tabular}{|c|c|c|c|}
\hline Item & Quantity & Part Name and Source & Cost \\
\hline $\begin{array}{l}\text { Plastic Syringes with Luer } \\
\text { Lock Connection* }\end{array}$ & $\begin{array}{l}1 \times 20 \mathrm{ml} \\
1 \times 30 \mathrm{ml} \\
1 \times 50 \mathrm{ml}\end{array}$ & $\begin{array}{l}\text { McMaster-Carr \#7510A45, } \\
\quad \text { \#7510A46. \#7510A656 }\end{array}$ & $\$ 6.00$ \\
\hline 2" needles with Luer Lock ${ }^{*}$ & 2 & McMaster-Carr \#75165A265 & $\$ 3.40$ \\
\hline Rubber stoppers* & $\begin{array}{l}1 \times \operatorname{size} 0 \\
1 \times \operatorname{size} 4 \\
1 \times \operatorname{size} 6\end{array}$ & $\begin{array}{l}\text { Karter Scientific \#216K2, } \\
\quad \text { \#216O2, and \#216Q2 }\end{array}$ & $\$ 3.66$ \\
\hline $\begin{array}{c}\text { 1/8" ID } 1 / 4 " \text { OD Tygon } \\
\text { Tubing* }\end{array}$ & 10 meters & McMaster-Carr \#6516T14 & $\$ 9.50$ \\
\hline $\begin{array}{l}\text { Luer lock tube fittings for } \\
1 / 8 \text { " ID tubing }\end{array}$ & $\begin{array}{c}10 \\
\text { female, } \\
10 \text { male }\end{array}$ & $\begin{array}{l}\text { McMaster-Carr \#51525K293, } \\
\quad \# 51525 \mathrm{~K} 143\end{array}$ & $\$ 11.35$ \\
\hline $\begin{array}{l}\text { Barbed tube fittings for } 1 / 8^{\prime \prime} \\
\text { ID tubing }\end{array}$ & 10 & McMaster-Carr \# 5463K626 & $\$ 8.97$ \\
\hline $\mathrm{CO}_{2}$ absorbent & $\begin{array}{l}100 \\
\text { grams }\end{array}$ & $\begin{array}{c}\text { Litholyme, Allied Heahtcare } \\
\text { Products Inc. }\end{array}$ & $\$ 2.00$ \\
\hline 5V-6VDC Mini Air Pump & 2 & $\begin{array}{c}\text { TCS Electrical Motor Co. } \\
\text { \#JQB2438274 }\end{array}$ & $\$ 17.98$ \\
\hline $\begin{array}{c}\text { 5V, 2.5A Step-Down Voltage } \\
\text { Regulator }\end{array}$ & 2 & Pololu product $\# 2858$ & $\$ 17.90$ \\
\hline $\begin{array}{c}\text { XP1500/XP2200 Peristaltic } \\
\text { Pump }\end{array}$ & 2 & AGO & $\$ 198.00$ \\
\hline $\begin{array}{l}\text { 12V Step-Up/Step-Down } \\
\text { Voltage Regulator }\end{array}$ & 2 & Pololu product $\# 2577$ & $\$ 29.90$ \\
\hline Air stone* & 1 & Pawfly \#ASC-030 & $\$ 0.70$ \\
\hline $\begin{array}{l}\text { Nylon syringe filter, Pore Size } \\
0.22 \mu \mathrm{m}^{*}\end{array}$ & 1 & AllPure \#AP-NY025N022 & $\$ 0.65$ \\
\hline Analog flow meter and valve & 1 & $\begin{array}{l}\text { JIAWANSHUN Oxygen Air Flow } \\
\text { Meter } 0.1-1.5 \text { LPM }\end{array}$ & $\$ 17.99$ \\
\hline \multirow[t]{2}{*}{ Plastic 14-inch toolbox } & 1 & $\begin{array}{l}\text { Akro-Mils 09514CFT ProBox } \\
\text { 14-Inch Plastic Storage Toolbox }\end{array}$ & $\$ 19.99$ \\
\hline & \multicolumn{2}{|r|}{ Gas Extractor Cost } & $\$ 348$ \\
\hline K30 10,000 ppm $\mathrm{CO}_{2}$ sensor & 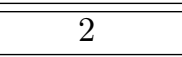 & CO2 Meter \#SE-0018 & $\$ 170.00$ \\
\hline ESP32 Development Board & 1 & $\begin{array}{l}\text { Mouser \#356-ESP32- } \\
\text { DEVKITC32D }\end{array}$ & $\$ 10.00$ \\
\hline $\begin{array}{l}\text { Waterproof Junction Box for } \\
\text { Electronics }\end{array}$ & 1 & $\begin{array}{l}\text { Waterproof IP65 Junction Box } \\
(150 \times 100 \times 70 \mathrm{~mm})\end{array}$ & $\$ 13.99$ \\
\hline $\begin{array}{l}\text { Waterproof Junction Box for } \\
\text { Electronics }\end{array}$ & 1 & $\begin{array}{l}\text { LeMotech Waterproof IP65 } \\
\text { Junction Box Enclosure Pale Gray } \\
3.3 " \text { x } 2.3 " \text { x } 1.3 "\end{array}$ & $\$ 7.99$ \\
\hline \multirow[t]{2}{*}{$\begin{array}{l}\text { Waterproof Junction Box for } \\
\text { Electronics }\end{array}$} & \multirow[t]{2}{*}{1} & $\begin{array}{l}\text { FX Waterproof IP68 } 3 \text { Way Plug } \\
\text { Line Power Cord Junction Boxes }\end{array}$ & $\$ 10.95$ \\
\hline & & Sensor Payload Cost & $\$ 213$ \\
\hline $\begin{array}{l}\text { Waterproof Junction Box for } \\
\text { Electronics }\end{array}$ & 1 & $\begin{array}{l}\text { Waterproof IP65 Junction Box } \\
(150 \times 100 \times 70 \mathrm{~mm})\end{array}$ & $\$ 13.99$ \\
\hline \multirow[t]{3}{*}{ Push Button Switch } & \multirow{2}{*}{\multicolumn{2}{|c|}{$\begin{array}{l}\text { TARELO 16MM 5/8" Latching } \\
\text { Push Button Switch 5A 12V } \\
\text { Power Routing Cost }\end{array}$}} & $\$ 21.70$ \\
\hline & & & $\$ 36$ \\
\hline & & Total Cost & $\$ \$ 597$ \\
\hline
\end{tabular}

Table 2.2: Parts list and cost of the LC-GEMS, broken into gas extraction, sensor payload, and power routing sub-systems. * Based on FaRAGE system construction [69] 
sensor inlet while grounding the switch input to execute the zero calibration operation code.

A calibration was run for 10 minutes, flowing varied concentrations of $\mathrm{CO}_{2}$ gas (225 ppm - $1000 \mathrm{ppm})$, varying $\mathrm{T}\left(20^{\circ} \mathrm{C}-26^{\circ} \mathrm{C}\right)$ and $\mathrm{H}(21 \%$ - $75 \%)$ while maintaining atmospheric pressure conditions. A LI-COR LI-810 $\mathrm{CO}_{2}$ sensor (LI-COR Biosciences, Lincoln, NE USA) was run simultaneously, capturing the ground truth $\mathrm{CO}_{2}$ measurement. The LI-COR is a high-precision commercial gas analyzer used to obtain the $\mathrm{CO}_{2, p p m}$ measurement for the multivariate linear regression model. These data were then used to create the multivariate linear regression model (Figure 2-8). Prior to the use of a multivariate linear regression model, the RMSE of the dissolved gas and sweep gas K30 sensors were 20.3 and 24.7 respectively. After use of the multivariate linear regression model, the RMSE of the dissolved gas and sweep gas K30 sensors were 3.0 and 3.2 respectively. This model is used going forward for all K30 readings in LC-GEMS measurements.

\subsection{LC-GEMS Calibrations and Characterization}

\subsubsection{LC-GEMS Gas Extraction Calibration}

Perfect equilibrium between the flowing water and flowing headspace is not achieved or expected with the LC-GEMS. Instead, the steady state measurement of the LCGEMS represents a partial equilibrium measurement, resulting in a raw measurement of gas concentration that is lower than expected (because the injected sweep gas dilutes any sample that is above the $\mathrm{CO}_{2}$ concentration in the sweep gas). The relationship between the LC-GEMS measurement and the true dissolved gas concentration must be known in order to translate its raw data to true measurements. This was measured experimentally by conducting lab calibrations, creating water samples of known dissolved gas content and running them through the LC-GEMS.

Samples of known dissolved gas concentration were made using a showerhead equilibrator set-up housed inside a sealed canister (Figure 2-9B). It should be noted that 

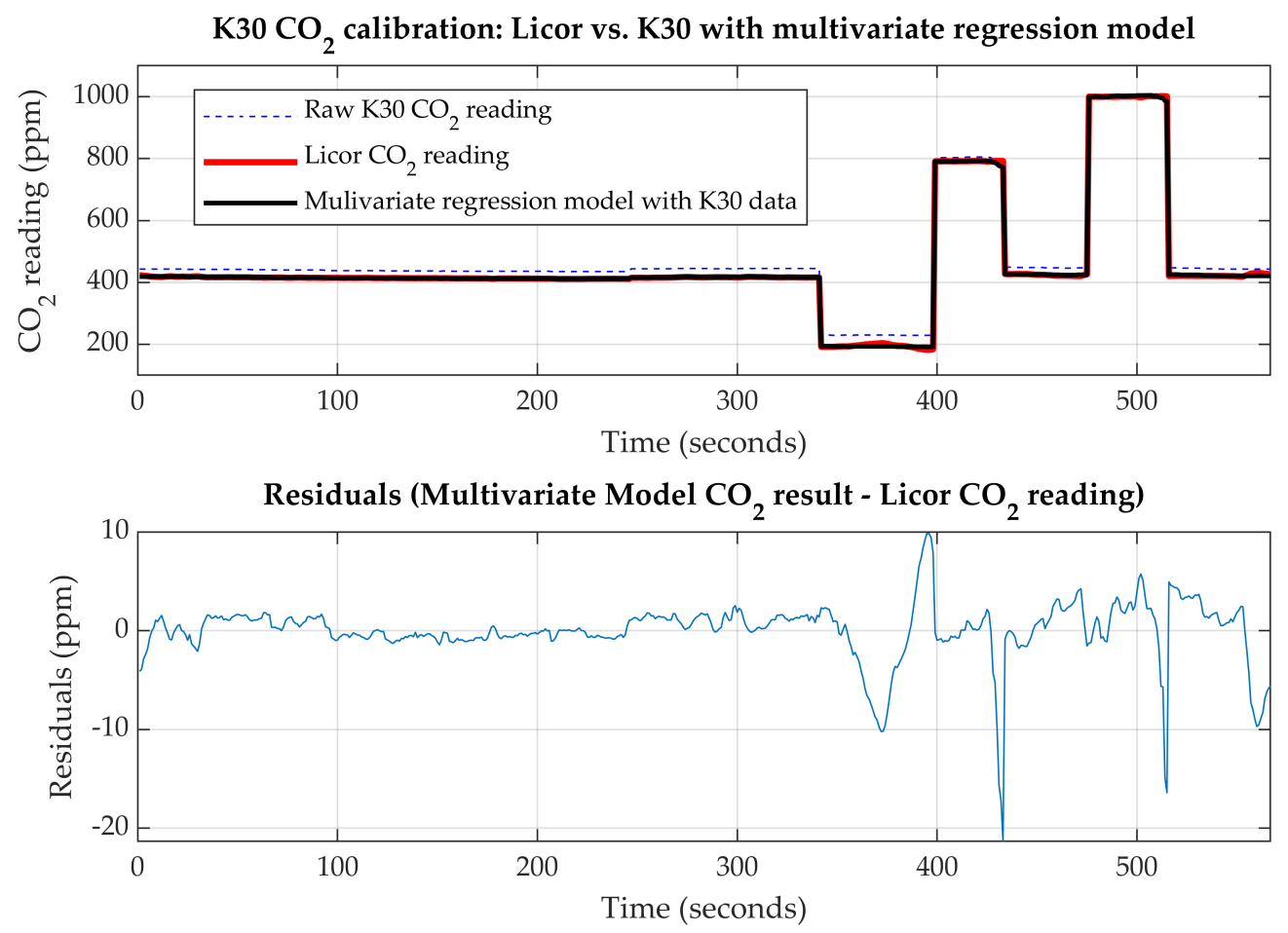

Figure 2-8: The results of fitting a multivariate linear regression model to the K30 $\mathrm{CO}_{2}$ sensor via a calibration with the LI-COR LI-810.

to control lab conditions, the canister was submerged in a temperature controlled sink (Figure 2-10) which was maintained at $15-20^{\circ} \mathrm{C}$ and the temperature of each water sample was recorded. A range of $\mathrm{CO}_{2}$ concentrations were first injected into the headspace via the gas ports. The headspace of the canister was then connected to the inlet and outlet ports of a LI-COR LI-810 (Figure 2-9). The showerhead pump was turned on and allowed to run until the LI-COR demonstrated a steady measurement after approximately 30-45 minutes, signaling that the headspace and water were totally equalibrated (an example of the LI-COR measuring the equilibration of the canister is shown in Figure 2-11).

The $\mathrm{CO}_{2}$ concentration in the headspace was recorded by the LI-COR in parts per million by volume. The LI-COR was then disconnected from the headspace and the water inlet of the LC-GEMS was connected to the canister. The LC-GEMS was then turned on and water from the canister was pumped to the inlet for approximately 2-3 

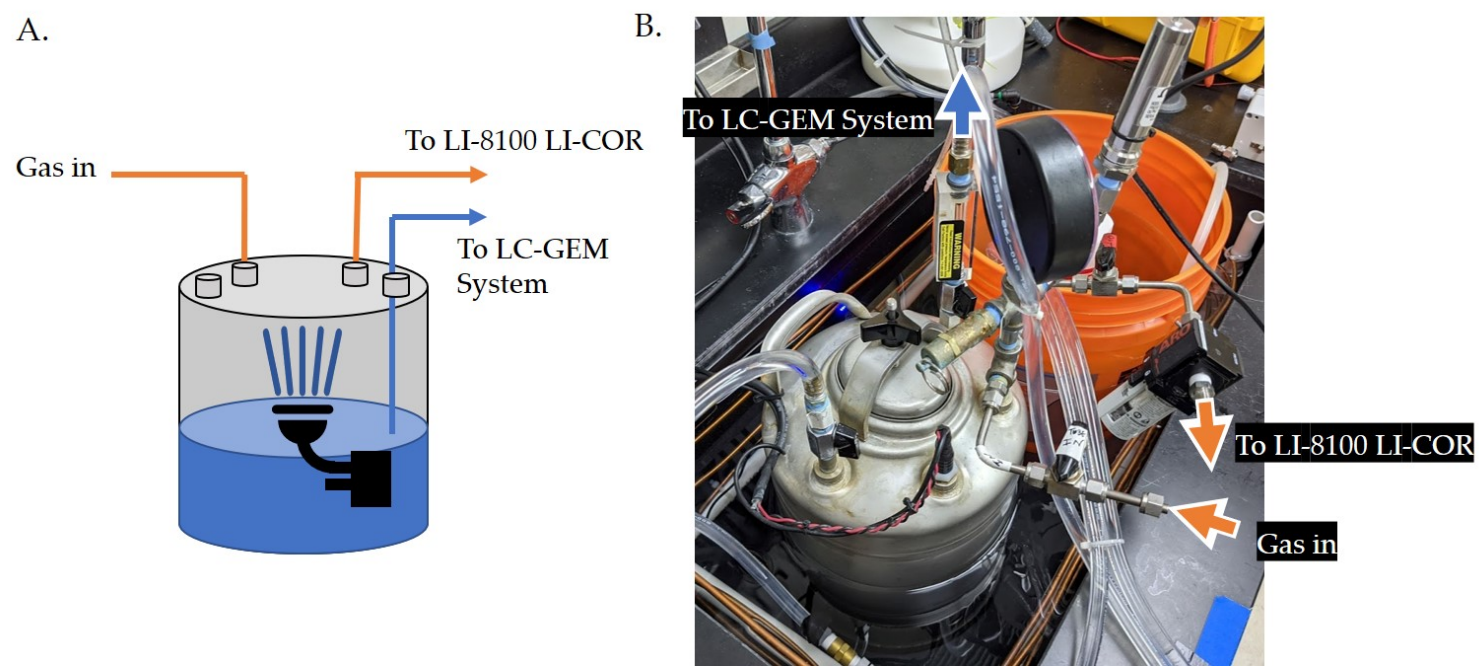

Figure 2-9: The showerhead equilibrator is illustrated in (A) and photographed in (B). A water pump and shower head attachment were inside a gas-tight canister and sprayed the sample water into the headspace. To adjust the $\mathrm{CO}_{2}$ concentration, gas was injected in the headspace through the gas inlet. To monitor the concentration and confirm that equilibration was achieved between the headspace and water, the LI-COR circulated the headspace by connecting its inlet and outlet.

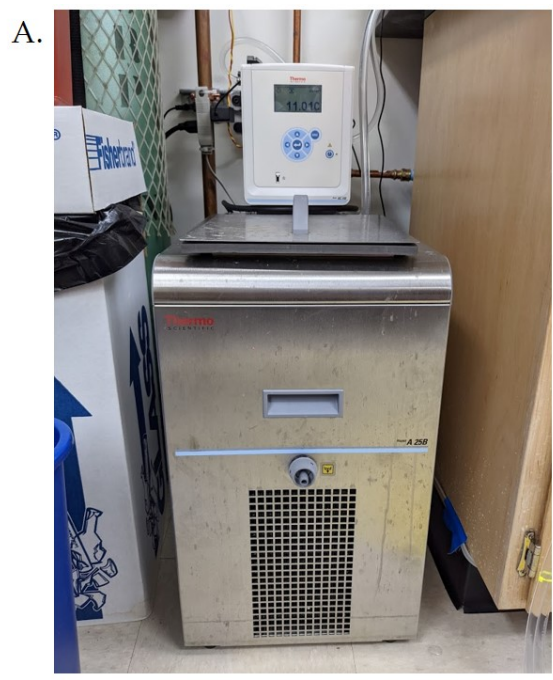

Water temperature controller

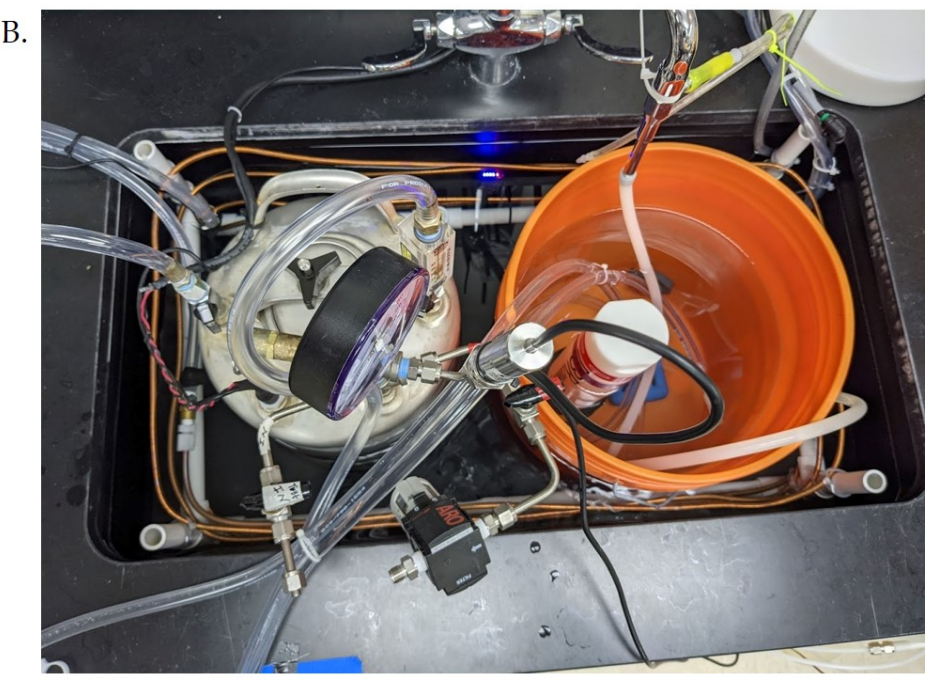

Copper tubing circulates temperaturecontrolled water in sink bath

Figure 2-10: (A) The water temperature control unit (Thermo Scientific AC150-A25B 13-21L Circulating Bath, Thermo Fisher Scientific, Waltham, MA, USA). (B) The temperature controlled water is pumped through the copper tubing in the sink bath. 


\section{LI-COR Showerhead Equilibration}

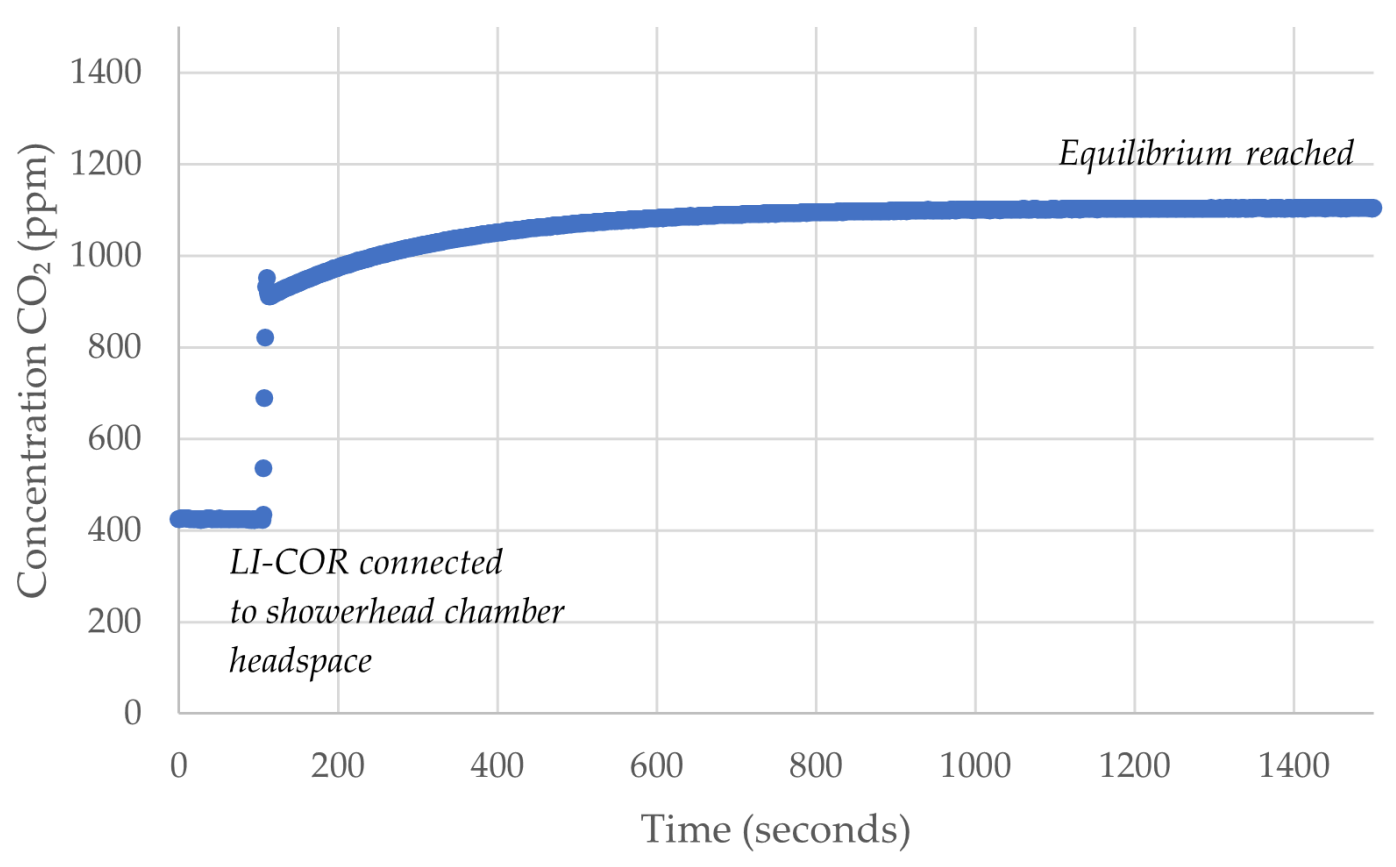

Figure 2-11: An example of the LI-COR measuring the headspace $\mathrm{CO}_{2}$ concentration in the showerhead canister. Once a steady state LI-COR reading is reached, the headspace and water are at equal concentrations of $\mathrm{CO}_{2}$. 
minutes, allowing the LC-GEMS to reach a steady state measurement (LC-GEMS time response is discussed in Section 2.5.2 and shown in Figure 2-15).

Calibrations were run for both fresh and saltwater samples. Salinity and gas solubility have an inverse relationship, meaning water with higher salinity will hold less gas [89]. Additionally, reduced $\mathrm{CO}_{2}$ desorption [90] from saltwater has previously been attributed to the differing ionization fractions of inorganic carbon content in fresh versus saltwater [91]. For this reason, it was expected that the LC-GEMS extraction efficiency would vary to some degree for fresh versus saltwater.

The LC-GEMS was run on 15 different samples of known $\mathrm{CO}_{2}$ concentration with freshwater (from a lab sink faucet) and 8 known samples of ocean saltwater (collected from a dock on Great Harbor in Woods Hole, MA). A calibration range of 400 ppm - 1500 ppm $\mathrm{CO}_{2}$ was used, to cover the range of atmospheric $\mathrm{CO}_{2}$ concentration to typical levels expected at the surface of lakes and ponds which are supersaturated with $\mathrm{CO}_{2}$ (1200 ppm) [57]. The surface concentration of $\mathrm{CO}_{2}$ in estuaries and coastal regions can reach as high as 12,000 ppm in the late summer and while this thesis did not calibrate for these conditions, future experiments could easily expand the LC-GEMS to that range [92]. The raw calibration data are plotted in Figure 2-12. Recall that the raw LC-GEMS measurement is the net extracted gas: the difference between the $\mathrm{CO}_{2}$ concentration of the input sweep gas and the LC-GEMS gas outlet.

It is visibly clear from the collected calibration data (Figure 2-12) that a linear relationship exists between the LC-GEMS and the predicted true $\mathrm{pCO}_{2}$ for this range of $\mathrm{CO}_{2}$ concentrations. The following statistical methods for error and confidence analysis are implemented to determine the accuracy of the LC-GEMS [93].

Figure 2-12 shows a simple linear regression model fit to the calibration data for salt and freshwater. However, better interpretations of the LC-GEMS reading were obtained when temperature was accounted for in the model. It is expected that extraction efficiency will vary with water temperature. Thus, a multivariate linear regression model was fit to the salt and freshwater calibration data, accounting for the independent variables of raw LC-GEMS reading and temperature of the sample water in determining the dependent variable, true $\mathrm{pCO}_{2}$. The regression coefficients 


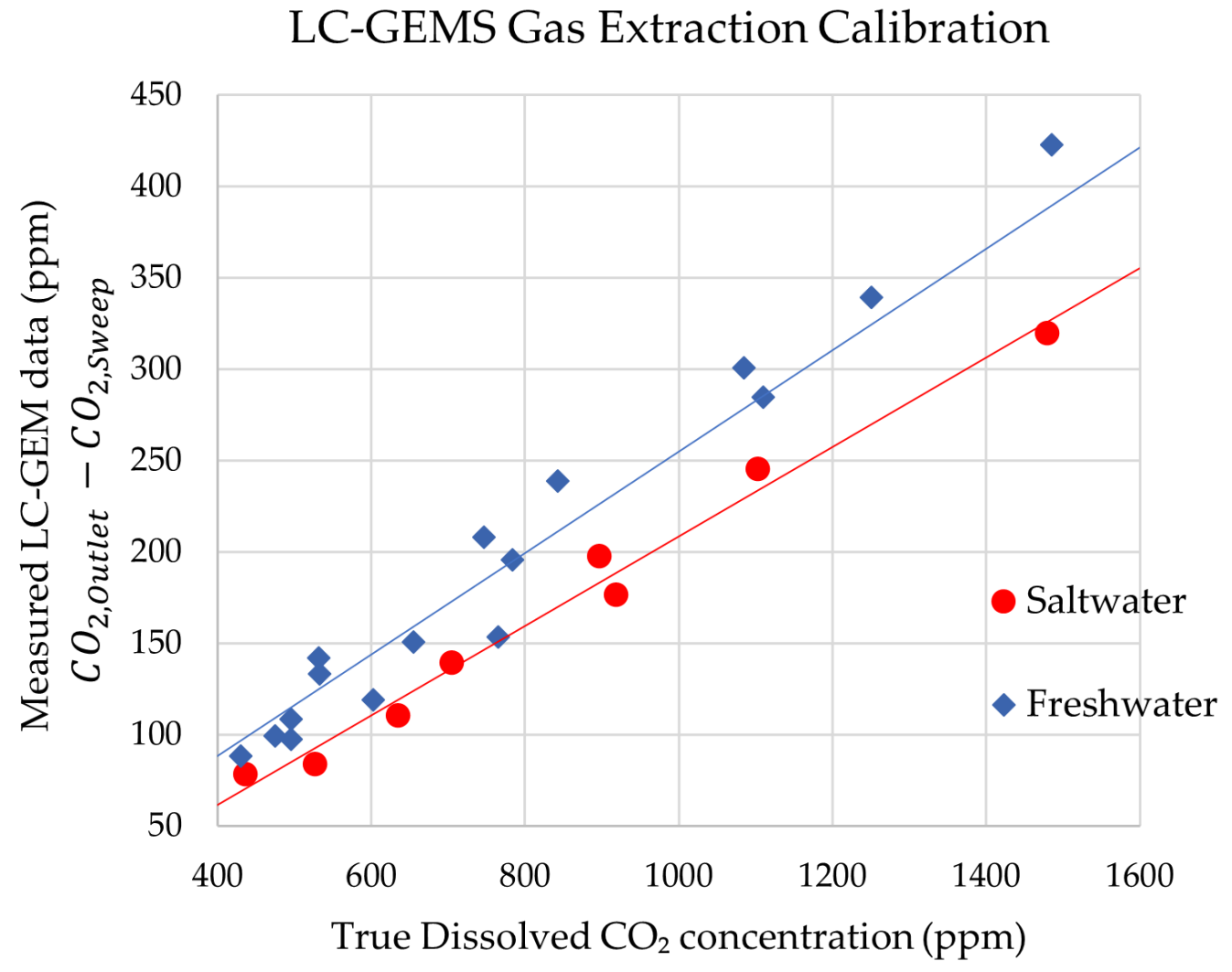

Figure 2-12: The raw calibration measurements for the LC-GEMS, with fresh (blue diamonds) and salt (red circles) water samples. A simple linear regression is plotted, relating the raw LC-GEMS measurement to the true dissolved $\mathrm{CO}_{2}$ content. However, Figure 2-13 shows more accurate multivariate linear regression models, which account for the temperature of the sample water. While the saltwater samples were maintained at a constant $15-16{ }^{\circ} \mathrm{C}$ temperature during calibration trials, the freshwater samples were run at a range of $15-20^{\circ} \mathrm{C}$ and more accurately show how temperature affects the extraction efficiency of the LC-GEMS. 
$\hat{\beta}_{0}$ (the intercept), $\hat{\beta}_{1}$ (the term for the relationship with the raw LC-GEMS reading, $\mathrm{x}$ ), and $\hat{\beta}_{2}$ (the term for the relationship with temperature, $\mathrm{T}$ ), were determined to solve for the predicted $\mathrm{pCO}_{2}$ concentration $(\hat{y})$. The hats $\left(^{\wedge}\right)$ designate that these terms are least squares estimates based on the calibration data in Figure 2-12. The estimated regression line is

$$
\hat{y}=\hat{\beta}_{0}+\hat{\beta}_{1} x+\hat{\beta}_{2} T
$$

The resulting multivariate linear models for the fresh and saltwater calibrations are plotted in Figure 2-13 and the regression coefficients are shown. In order to visualize the behavior of a multivariate model, one independent variable, in this case temperature, must be held constant across the range of possible LC-GEMS readings. The larger $\hat{\beta}_{1}$ term for the saltwater model reflects that the LC-GEMS is less efficient at extracting gas in saltwater, as predicted. The large difference between $\hat{\beta}_{0}$ and $\hat{\beta}_{2}$ for the saltwater and freshwater models is attributed to the fact that the seawater data were collected for a very small range of temperature and over a smaller sample size. For this reason, the saltwater calibration model does not accurately reflect the temperature dependency of the LC-GEMS. It is expected from these calibration results that temperature has a much greater affect on the extraction efficiency of the LC-GEMS than the sample water salinity does. Moving forward, the freshwater gas extraction calibration model is used for all LC-GEMS data. Future calibrations with a larger and more varied range of temperatures with allow for multivariate models that account for not only temperature and LC-GEMS reading but also relative salinity.

From the freshwater calibration data and linear multivariate regressions model, a confidence interval on the mean response at any value $\mathrm{x}$ can be found. At a single LC-GEMS measurement, $x_{0}$, a $100(1-\alpha) \%$ confidence interval for $\widehat{p C O}_{2,0}$ is expressed by

$$
\hat{y}_{0} \pm t_{(\alpha / 2, n-2)} \sqrt{M S_{R e s} \cdot X_{0}^{T}\left(X^{T} X\right)^{-1} X_{0}}
$$

The term $t_{(\alpha / 2, n-2)}$ is the $\mathrm{T}$ distribution, for which a $95 \%$ confidence interval has 
A.

\section{LC-GEMS Freshwater Gas Extraction Calibration}

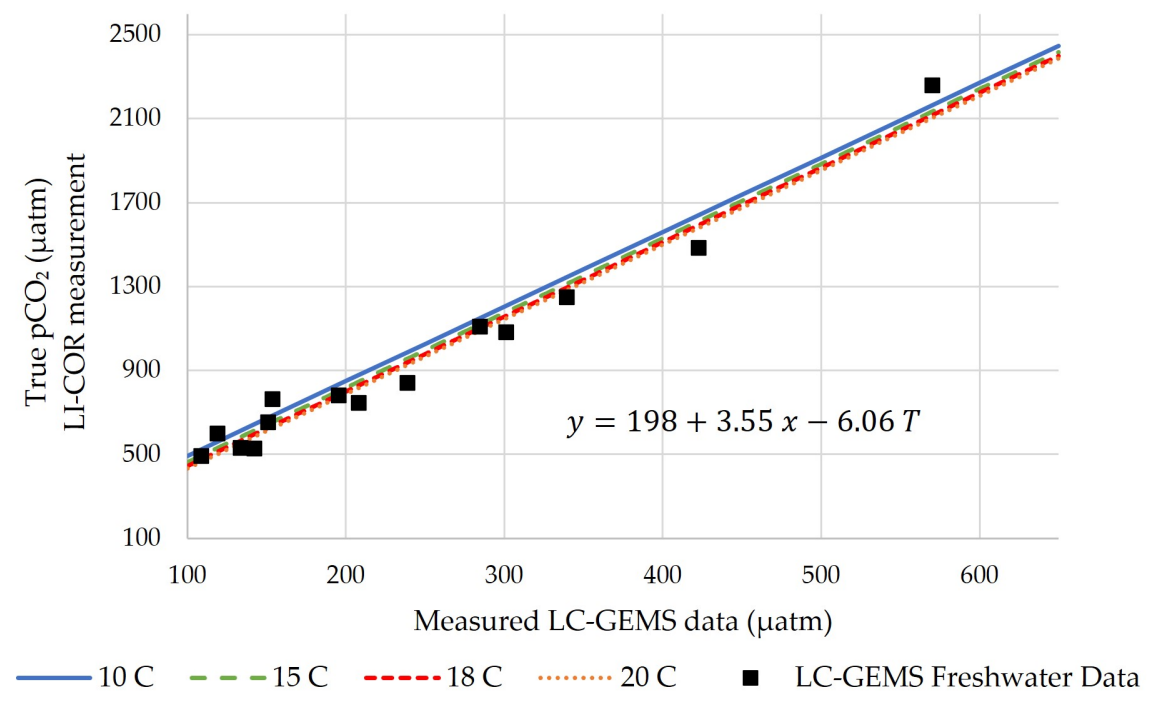

B.

\section{LC-GEMS Saltwater Gas Extraction Calibration}

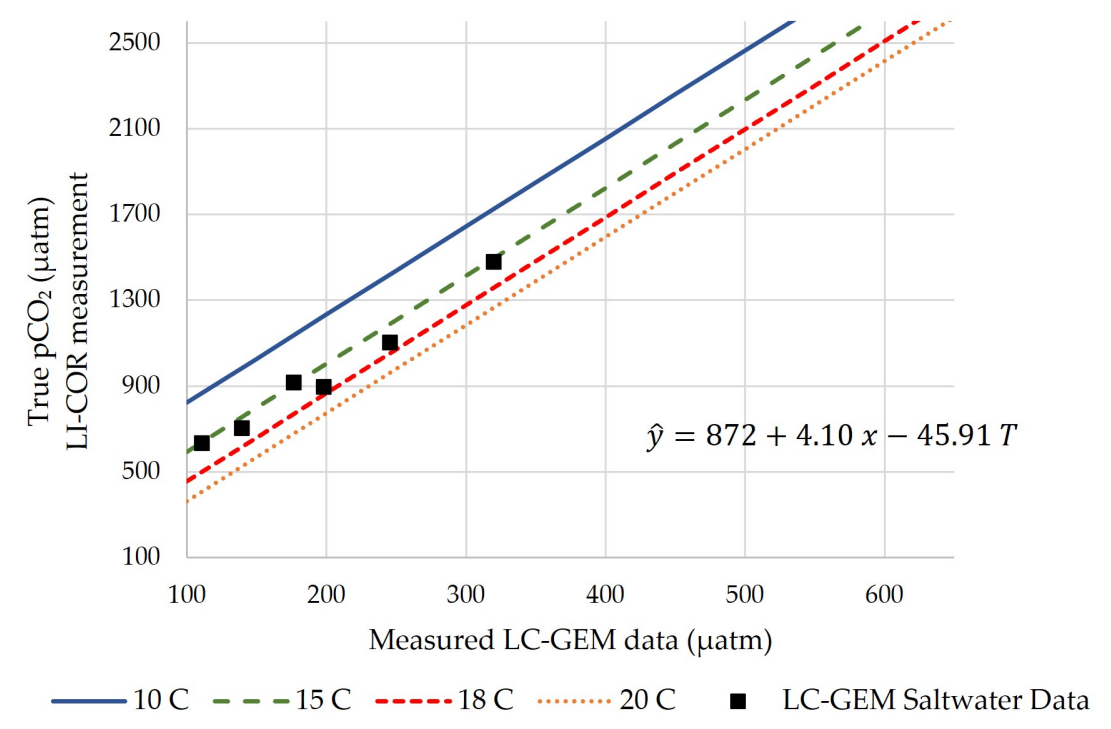

Figure 2-13: Gas extraction calibration plots for LC-GEMS with (A) fresh and (B) saltwater. The best fit linear multivariate regression equations are shown and give a conversion equation between the LC-GEMS extraction reading, $(\mathrm{x}=$ $\mathrm{CO}_{2, \text { Outlet }}-\mathrm{CO}_{2, \text { Sweep }}$ ), sample water temperature (T), and the dissolved $\mathrm{CO}_{2}$ concentration (y). The conversion is plotted for different theoretical water sample temperatures between $10-20^{\circ} \mathrm{C}$. The difference in the expressions for fresh and saltwater are in part attributed to the differences in salinity but seem to be primarily a result in the limited temperature range $\left(15-16^{\circ} \mathrm{C}\right)$ used in the saltwater equilibration calibration experiments as compared to the range used for the freshwater calibration experiments $\left(15-20^{\circ} \mathrm{C}\right)$. For this reason the freshwater calibration is used for LC-GEMS measurements in this study. See Figure 2-14 for confidence and prediction intervals of the LC-GEMS. 


\section{LC-GEM System Confidence and Prediction Intervals}

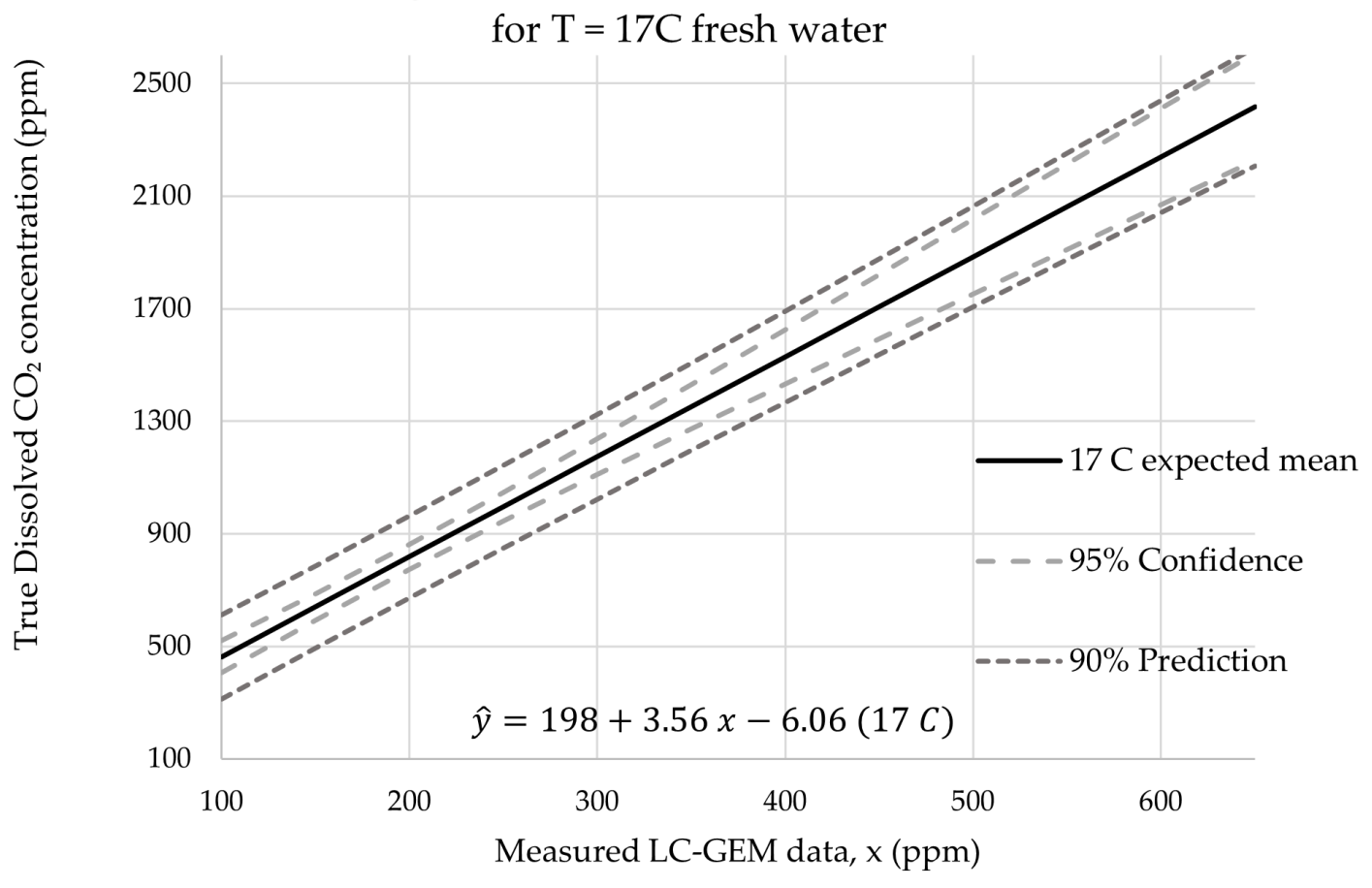

Figure 2-14: Confidence and prediction intervals for LC-GEMS as calculated from Equations 2.5 and 2.6 respectively. The black line plots the expected true dissolved $\mathrm{CO}_{2}$ concentration from measured LC-GEMS data at a constant $17^{\circ} \mathrm{C}$ using the multivariate linear regression model built from the freshwater calibration data. The $95 \%$ confidence interval gives us confidence limits on the value of $\mathrm{CO}_{2}$ predicted from the LC-GEMS calibration. The 90\% prediction intervals give a conservative estimate of the error bars for future field measurements, reflecting the uncertainty of the measurement due to systematic and random error. For all measurements with the LC-GEMs in this thesis, $90 \%$ prediction intervals will be used to ensure conservative measurements.

an $\alpha=0.025$. $M S_{\text {Res }}$ is the mean squared error, $X$ is the matrix that represents the LC-GEMS calibration data in the form $[x, T]$ (the raw LC-GEMS reading, $\mathrm{x}$, and the temperature reading, $T$ ) and $X_{0}$ is the newly collected data point ( $\mathrm{x}$ and $\mathrm{T}$ reading) for which $\hat{y}_{0}$ is being calculated [94]. This $95 \%$ confidence interval gives us confidence limits on the value of $\mathrm{CO}_{2}$ predicted from the LC-GEMS measurement in the calibration [93].

Another statistical tool, prediction intervals, gives more conservative limits on where to expect data for the LC-GEMS to lie in future measurements [94]. The 
$100(1-\alpha) \%$ prediction interval is calculated using the expression

$$
\hat{y}_{0} \pm t_{(\alpha / 2, n-2)} \sqrt{M S_{R e s}\left(1+X_{0}^{T}\left(X^{T} X\right)^{-1} X_{0}\right)}
$$

Both $95 \%$ confidence and prediction intervals are plotted for the LC-GEMS linear multivariate fit assuming $\mathrm{T}=17^{\circ} \mathrm{C}$ in Figure 2-14. This shows the expected accuracy of the LC-GEMS system at $17{ }^{\circ} \mathrm{C}$. Note that Figure 2-14 could be plotted for any temperature and that $17{ }^{\circ} \mathrm{C}$ was chosen for this graphical representation because it was approximately the average temperature used in the freshwater calibration trials.

For all measurements with the LC-GEMS in this thesis, 95\% confidences intervals will be used as a conservative estimate of the error bars for field measurements, reflecting the uncertainty of the measurement due to systematic and random error.

\subsubsection{LC-GEMS Time Response}

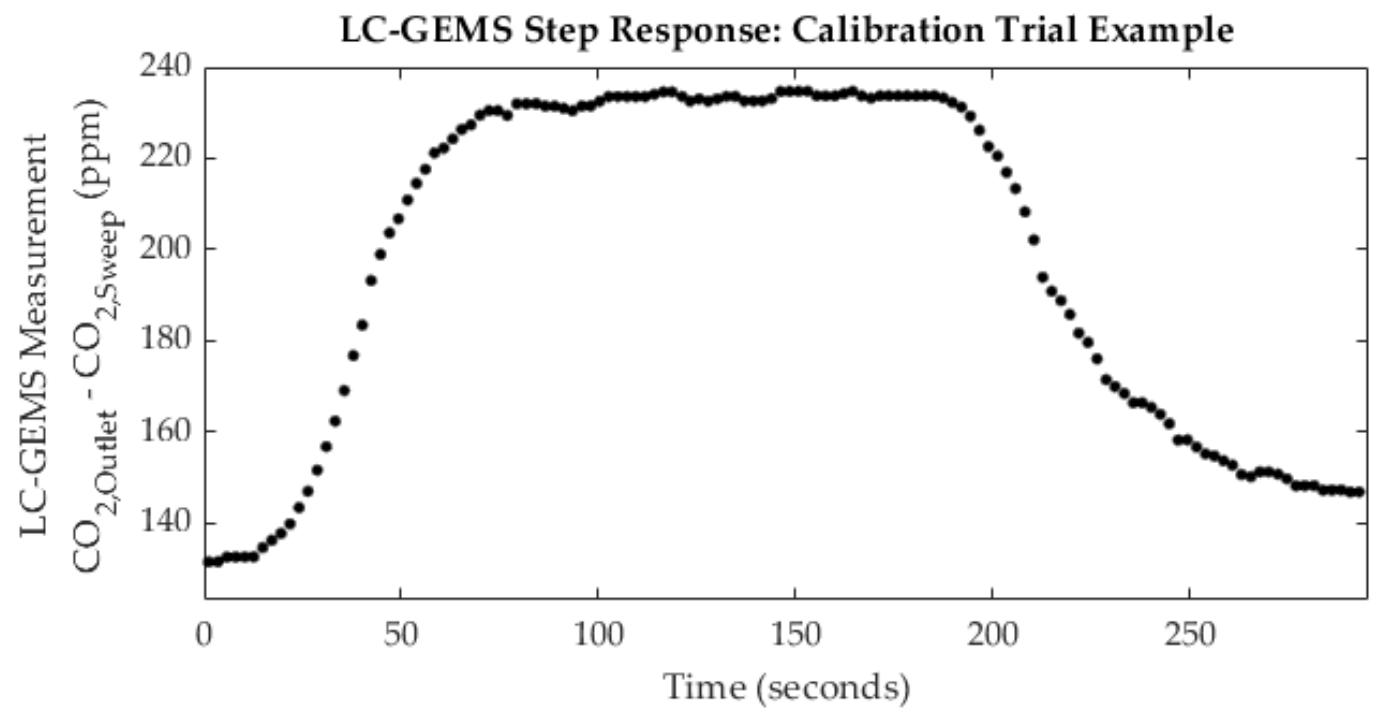

Figure 2-15: An example of the step response of the LC-GEMS. At time $\mathrm{t}=0$, LC-GEMS was measuring room equilibrated saltwater. The inlet of LC-GEMS was then moved to saltwater with a dissolved $\mathrm{CO}_{2}$ concentration of $897 \mathrm{ppm}$ and a steady state was reached with a low-to-high step response of $t_{63 \%}=34$ seconds. After reading a steady measurement, the inlet was moved to a different room equilibrated water sample and demonstrated a high-to-low step response of $t_{63 \%}=40$ seconds. See section 2.5.2 for discussion of LC-GEMS time response. 
For all the calibration trials run in Section 2.5.1, the time responses of the system were determined. The time response definition used here is $t_{63 \%}$, which represents the time is takes for the system to reach $63 \%$ of its final steady state reading from the initial step change. Here we report both response time for high-to-low concentrations and low-to-high concentrations, because efficiency of high-to-low equilibration is expected to be lower $[43,95]$. The average and standard deviation results for both fresh and saltwater are presented in Table 2.3. An increase for low-to-high $t_{63 \%}$ to high-to-low $t_{63 \%}$ is observed for freshwater but the two are relatively constant for salt water. The larger low-to-high $t_{63 \%}$ for saltwater matches the expectation that gas is more difficult to extract from saltwater. The average high-to-low $t_{63 \%}$ time responses of fresh and saltwater samples were 37 and 36 seconds respectively. This result shows that the LC-GEMS demonstrates a slightly slower time response than the FaRAGE, which is expected due to the decreased precision in flow accuracy and balance. However, compared to the state-of-the-art gas extraction technology, the LC-GEMS time response is among the fastest of those reported. Webb et al. (2016) reported time responses from 34 seconds - 2 minutes for marble, showerhead, and membrane type gas extractors. Of the techniques report in Webb et al. (2016) with similar time responses to the LC-GEMS, such as the large membrane contactor (3M Liqui-Cel), the LC-GEMS is comparatively much cheaper and more field-portable.

\begin{tabular}{|l|l|l|l|l|l|l|}
\hline & \multicolumn{3}{|c|}{$t_{63 \%}$ Low to High $\mathbf{C O}_{\mathbf{2}}$} & \multicolumn{3}{l|}{$t_{63 \%}$ High to Low $\mathbf{C O}_{2}$} \\
\hline Sample & Mean & StDev & N & Mean & StDev & N \\
\hline Freshwater & $28 \mathrm{~s}$ & $7 \mathrm{~s}$ & 17 & $37 \mathrm{~s}$ & $6 \mathrm{~s}$ & 13 \\
\hline Seawater & $37 \mathrm{~s}$ & $9 \mathrm{~s}$ & 5 & $36 \mathrm{~s}$ & $4 \mathrm{~s}$ & 5 \\
\hline
\end{tabular}

Table 2.3: Time constant, $t_{63 \%}$, for fresh and saltwater samples, where $\mathrm{N}$ is the number of samples, going from low to high concentrations of dissolved $\mathrm{CO}_{2}$ and from high to low. The increase in between low-to-high $t_{63 \%}$ and high-to-low $t_{63 \%}$ is observed for freshwater but the two are relatively constant for salt water.

The higher time response in the current LC-GEMS as compared to the FaRAGE system is attributed to 1) the response time of the K30 sensor and 2) the time it takes to fill the gas analysis chamber that houses the K30 sensor. The response time of the K30 sensor is $t_{63 \%}=20$ seconds when gas diffuses through its sensing chamber, 
as compared to the 5 second response time of the GasScouter G4301 used in the FaRAGE system [69]. The gas analysis chamber for the K30 sensor, through which the extracted gas flows for analysis, was designed to house the K30, the BME-280 sensor, and other additional low-cost gas sensors. The chamber and headspace size can reduced to minimize the time it takes for the extracted gas to equilibrate within the analysis chamber. It is expected that further reduction of the gas analysis housing would reduce the response time.

\subsection{Field Measurements}

\subsubsection{Field site}

Once calibrated in the lab, the LC-GEMS was field tested at Little Sippewissett Marsh, located in eastern Buzzards Bay in Falmouth, Massachusetts (41.574698, 70.640739). A sample site was chosen to measure dissolved $\mathrm{CO}_{2}$ concentration at different times of the day on April 19th, 20th, and 28th, 2021 (Figure 2-16). These dates were chosen because they were relatively sunny and we expected to see changes in dissolved $\mathrm{CO}_{2}$ concentration due to microbial and photosynthetic activity. The site was selected in a relatively stagnant pool of the marsh (Figure 2-17) where the LC-GEMS inlet could rest at the marsh floor, approximately 4 to $30 \mathrm{~cm}$ from the surface throughout the day. The water averaged a salinity of approximately 31 PSU on all field testing days. The location can experience large tidal changes but does not flow at high velocities. On April 19th and 20th, the relative change between high and low tide of Woodneck beach, the location of the inlet to Little Sippewissett Marsh, was only 0.6 meters while on April 28th the relative change was approximately 1.5 meters. The bed of the marsh at this sample site was of fine silt and very muddy. Plant life, specifically grass, was visibly present at the site in and out of the water (Figure 2-17). This type of site was selected as opposed to the more active inlet and outlet channel of the marsh, where water is actively flowing and $\mathrm{CO}_{2}$ flux may be impacted by this motion. 


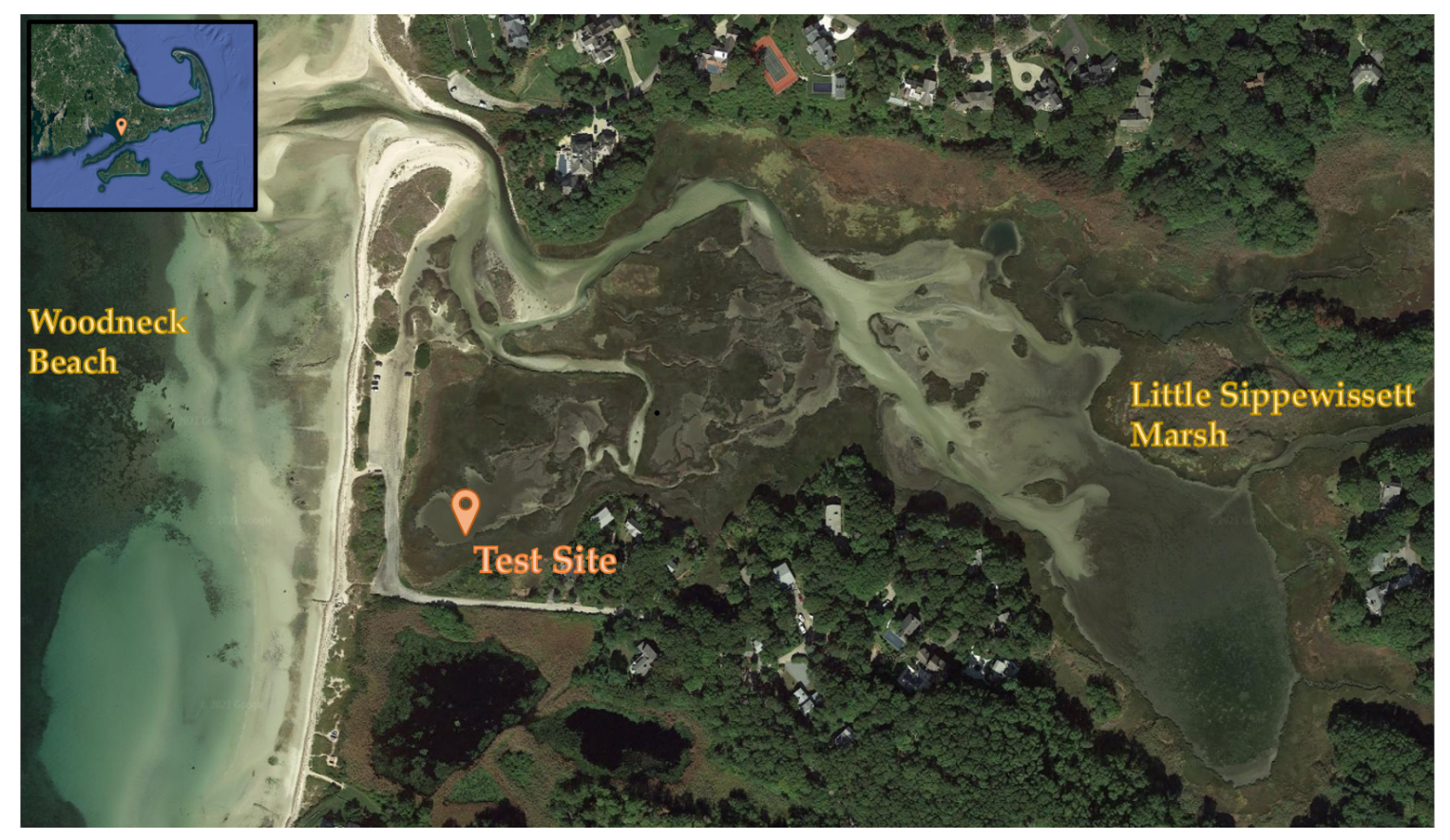

Figure 2-16: Satellite imagery of the Little Sippewissett Marsh field site, with the test site location identified. The site is in a relatively stagnant pool of the marsh, which can experience large tidal changes but does not flow at high velocities. In the top left corner, the location of the Little Sippewissett Marsh is identified along the inner coast of Massachusetts in Buzzards Bay. (C) 2021 MassGIS, Commonwealth of Massechusetts EOEA, Maxar Technologies, USDA Farm Service Agency, Map data (C) 2021

A.

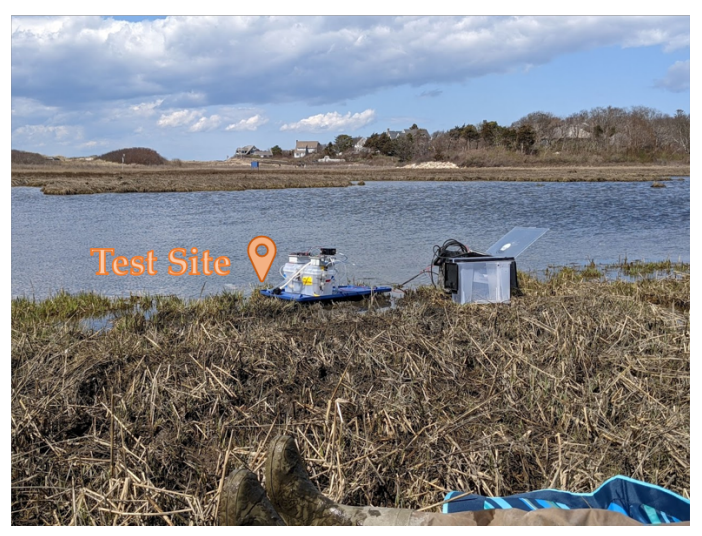

B.

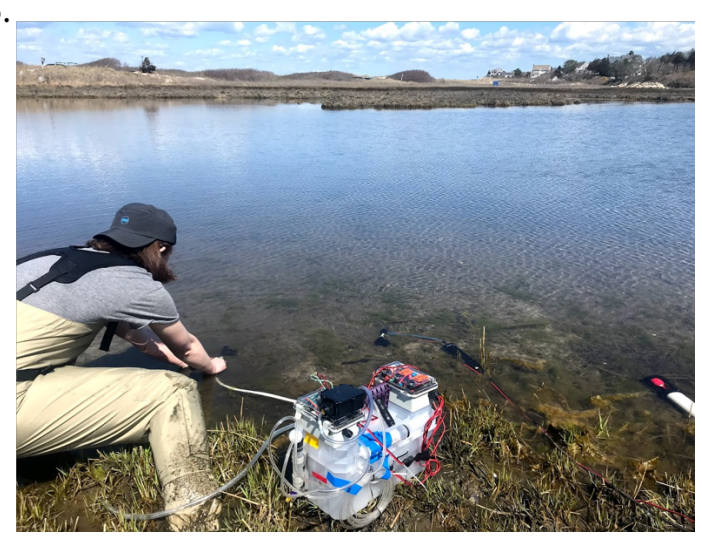

Figure 2-17: (A) Photograph of the field site sampling location. In this pool of the salt marsh, the water is relatively still. (B) The LC-GEMS sample water inlet being place into the marsh. 


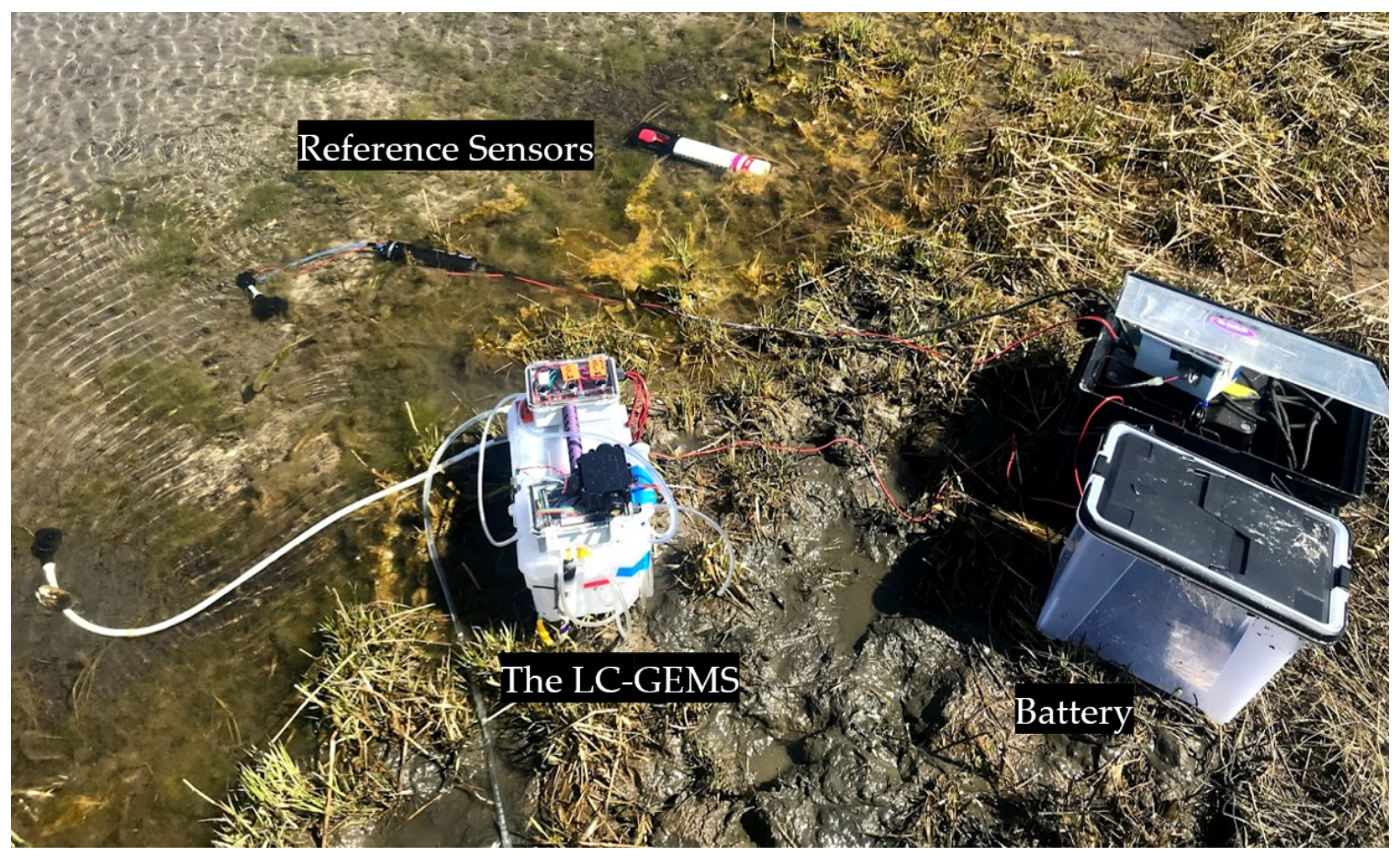

Figure 2-18: LC-GEMS making measurement in the field along side the C-sense reference sensor and the RBRconcerto CTD.

\subsubsection{Sampling Procedure}

The LC-GEMS was deployed at the water's edge (Figure 2-18). The inlet and outlet tubing of the LC-GEMS were placed carefully above the bed of the marsh to avoid disturbing the pond sediment. A mesh filter was added to the inlet tubing to ensure no organic material or dirt particulates would clog or interfere with the LC-GEMS.

The inlet and outlet water port of the LC-GEMS were spatially separated so that gas-extracted water did not interfere with the ambient water $\mathrm{CO}_{2}$ concentration. The system was powered by a Goal Zero Yeti 400 portable power station and pulled no more that 1.2 Watts on any deployment. On April 19th and 29th the system was deployed 8 times and run for 5 minutes, allowing for several minutes of constant measurement. On April 28th, the LC-GEMS was run 7 times from 20 minutes up to an hour to further evaluate its stability and robustness.

For the purpose of validating the LC-GEMS performance, a Turner Designs Csense sensor was used to measure $\mathrm{pCO}_{2}$ concentration simultaneously (Figure 2-19A). 
The C-sense is a commercial $\mathrm{pCO}_{2}$ sensor which works off NDIR detection. The Csense used had a full measurement scale of 2,000 ppm $\mathrm{CO}_{2}$ and a stated accuracy of 40ppm [96]. The C-sense was calibrated on April 10th, 2021, prior to the field testing. A $12 \mathrm{~V}$ pump flowed water across the C-sense membrane inlet and a mesh filter was used at the pump inlet, similar to the LC-GEMS tubing inlet. The C-sense $\mathrm{pCO}_{2}$ sensor is designed to output a voltage that can be translated to $\mathrm{ppm} \mathrm{CO}_{2}$ via a calibration equation provided by the manufacturer. A portable data-logger was constructed to $\log$ the $\mathrm{C}$-sense $\mathrm{CO}_{2}$ sensor data using an Arduino Uno and SD card data-logging shield and these electronics and the sensor itself was powered off of the same Goal Zero Yeti 400 portable power station as the LC-GEMS (Figure 2-19B). With a pump, total equilibration time of the $\mathrm{C}$-sense $\mathrm{CO}_{2}$ is approximately 12 minutes, so the sensor was left running for at least 15 minutes during each trial to ensure total equilibration was reached. On April 28th, the C-sense was run simultaneously with the LC-GEMS. A RBRconcerto CTD was placed in the water to monitor temperature, salinity, and depth (Figure 2-19C).

The LC-GEMS, C-sense, and RBRconcerto CTD were always deployed such that their inlets were as close as possible. Note that the recorded depth of the sensors is based on where the sensors could be positioned in the field at the time of deployment and does not always trend with relative tide level.

Data were collected from the test site at different times throughout the day, from early morning to evening. The light levels were collected from the public data logging of solar power generation at the Falmouth Landfill Solar Array, located 5 miles north east of the field site (data publically available via AlsoEnergy ${ }^{T M}$ ).

\subsubsection{Field Site Measurement Results}

The results of the LC-GEMS field testing data for April 19th-20th are plotted in Figure 2-20. The results of the LC-GEMS field testing data on April 28th are plotted in Figure 2-21, with an exemplary hour-long measurement plotted in Figure 2-22. Note that the LC-GEMS measures in the gas phase in ppm via the K30 sensor, so the raw measurements must be converted to partial pressure $\left(\mathrm{pCO}_{2}\right)$ via Equation 2.1. 


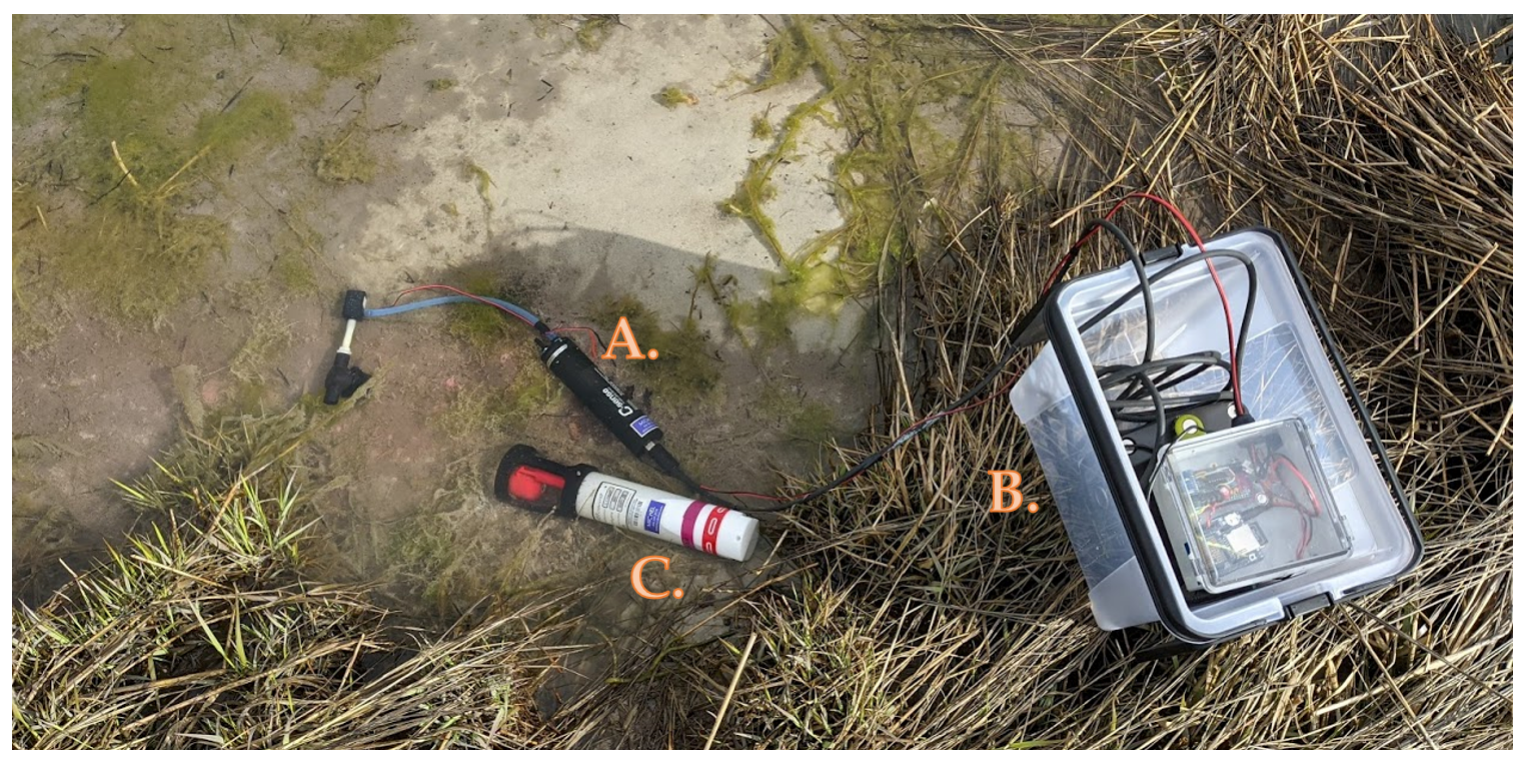

Figure 2-19: (A) The Turner Designs C-sense $\mathrm{pCO}_{2}$ sensor is pictured, attached to a $12 \mathrm{~V}$ pump which flows water across the C-sense membrane inlet. A mesh filter is used at the pump inlet to ensure no organic or dirt particulates reach the $\mathrm{C}$-sense membrane. The $\mathrm{C}$-sense is used as a reference $\mathrm{pCO}_{2}$ measurement to evaluate the performance of the LC-GEMS. (B) The electronics for powering and logging the Turner Designs C-sense $\mathrm{CO}_{2}$ sensor. (C) The RBRconcerto CTD is pictured. The CTD collects and logs temperature, salinity, and depth measurements. 


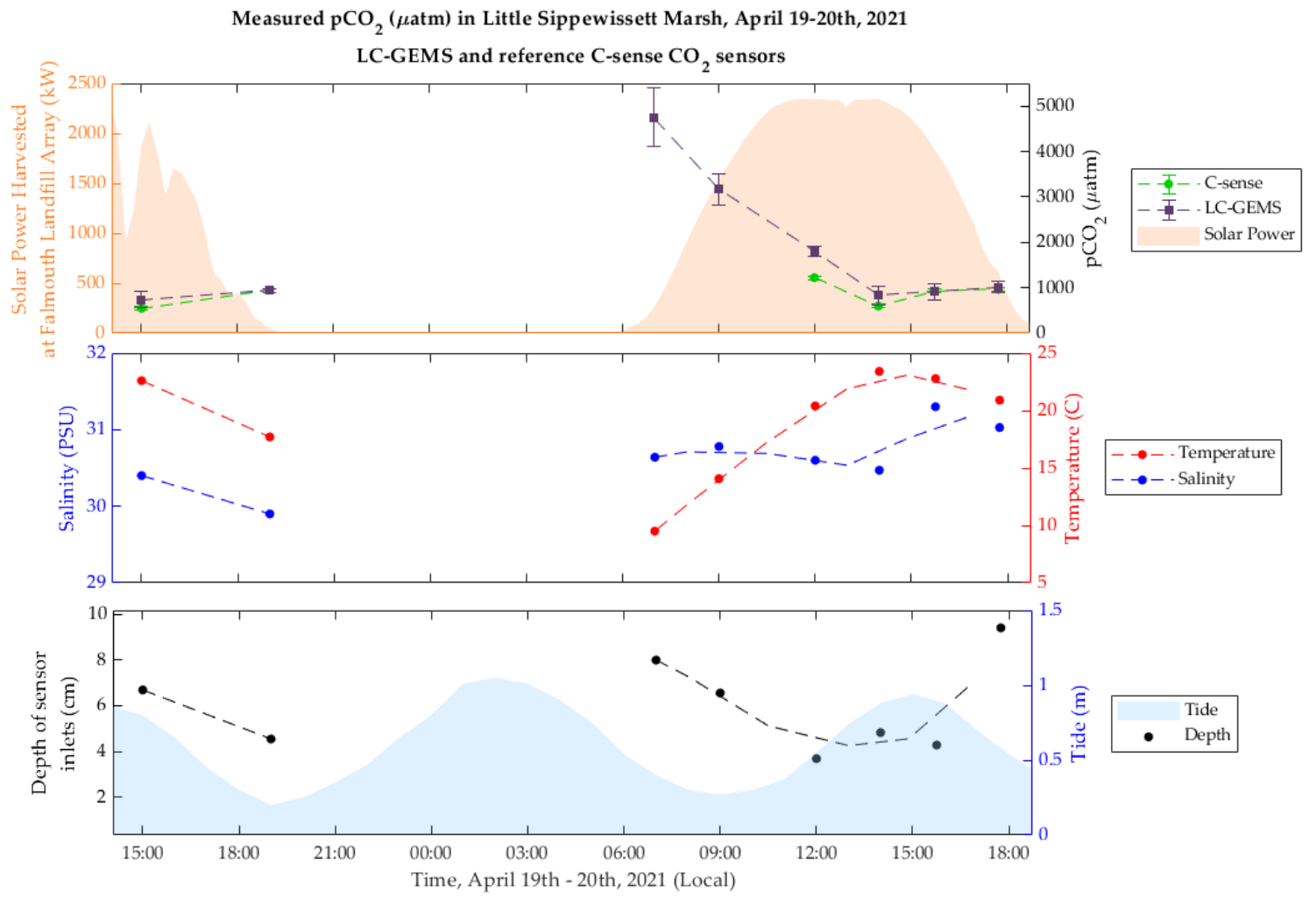

Figure 2-20: April 19th-20th, 2021, Field Work Data. Top: Each data point represents a 2 minute average reading from the C-sense and LC-GEMS sensor. The error bars for the LC-GEMS measurements are 95\% confidence intervals. The C-sense is listed as having a $40 \mathrm{ppm}$ accuracy, represented here in its error bars [96]. Note that for the data points at 07:00 and 09:00 the C-sense was railed-out at its maximum reading of $2000 \mathrm{ppm}$, so no C-sense reference data are available at those data points. Middle: The salinity and temperature readings from the RBRconerto CTD are plotted with dashed 2-point moving averages. Bottom: The tide of Woodneck Beach (which is the inlet to Little Sippewissett Marsh, shown in Figure 2-17) is plotted in light blue and supplied from www.tidetablechart.com. The depth of the sensors' inlets as measured by the RBRconcerto CTD are plotted in black along with a dashed 2-point moving average. The depth of the sensors is based on where the sensors could be positioned in the field at the time of deployment and does not always trend with relative tide level. 


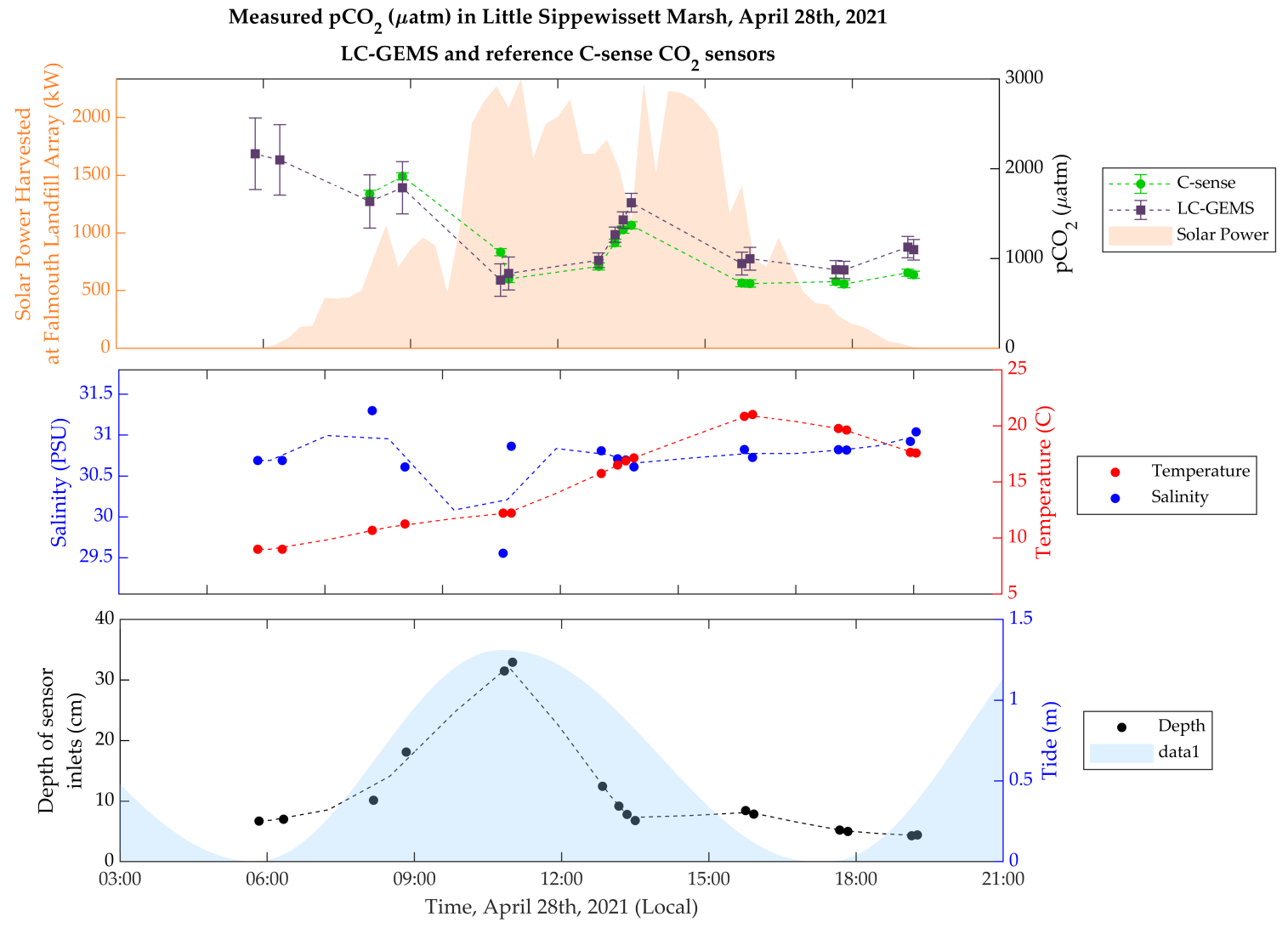

Figure 2-21: April 28th Field Work Data. Top: Each data point represents a 10 minute average reading from the C-sense and LC-GEMS sensors, note that this is a longer averaging than for the April 19th-20th data. The error bars for the LC-GEMS measurements are $95 \%$ confidence intervals. The C-sense is listed as having a 40 ppm accuracy, represented here in its error bars [96]. Note that around 06:00 the C-sense was railed-out at its maximum reading of $2000 \mathrm{ppm}$, so no reference data are available for the LC-GEMS at those data points. The solar power data reflects the light levels throughout the day. Middle: The salinity and temperature readings from the RBRconcerto CTD are plotted with dashed 2-point moving averages. Bottom: The depth of the sensors' inlets as measured by the RBRconcerto CTD are plotted in black along with a dashed 2-point moving average. The tide of Woodneck Beach (which is the inlet to Little Sippewissett Marsh, shown in Figure 2-17) is plotted in light blue and supplied from www.tidetablechart.com. The depth of the sensors is based on where the sensors could be positioned in the field at the time of deployment and does not always trend with relative tide level. 
All measurements were taken at less than $30 \mathrm{~cm}$ depth, so atmospheric TDGP was assumed to convert ppm directly to $\mathrm{pCO}_{2}$. In the results from both April 19th-20th and April 28th, the LC-GEMS clearly follows the trends measured by the C-sense.

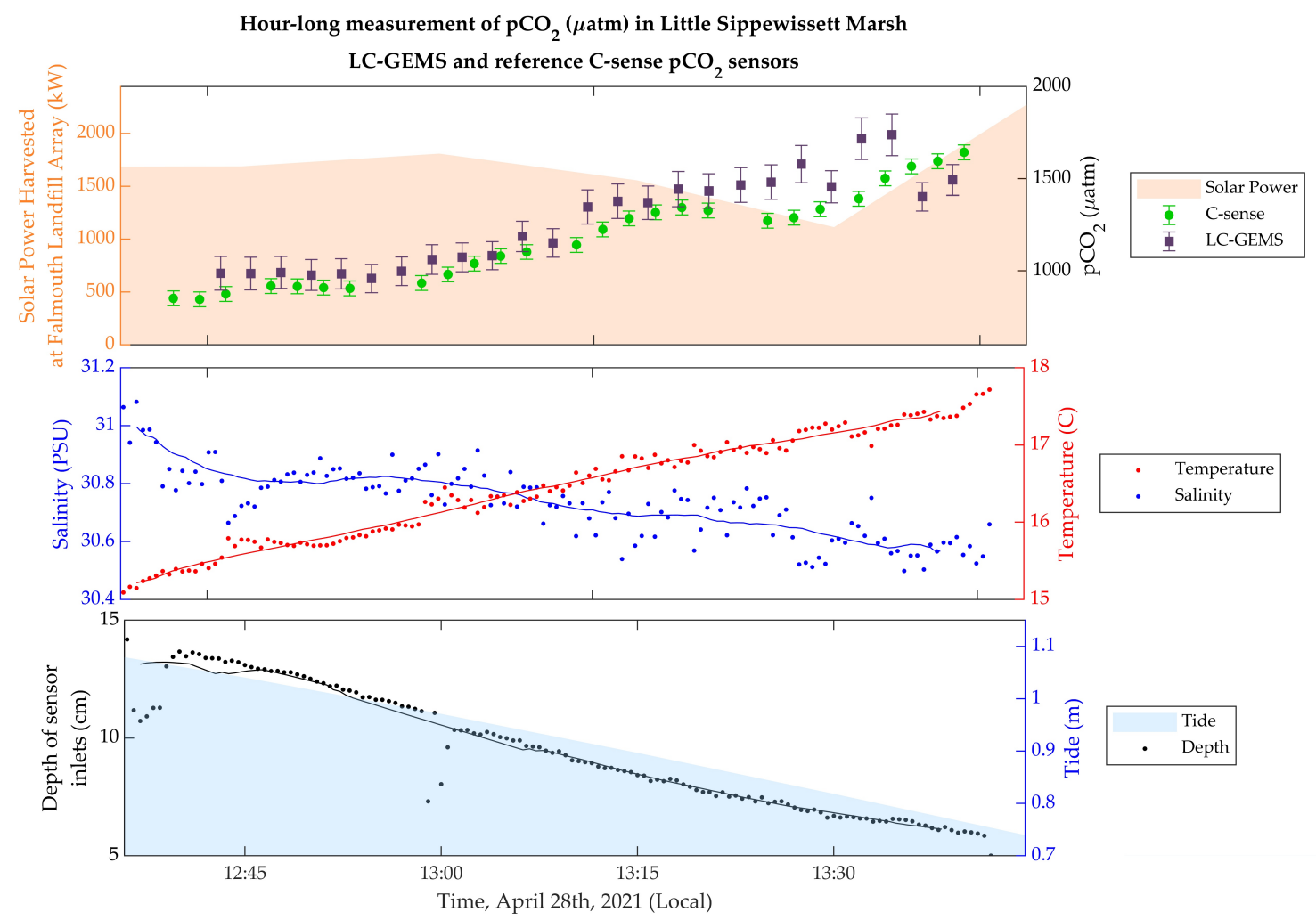

Figure 2-22: Hour long measurement with the LC-GEMS and C-sense starting at 12:40 on April 28th, 2021. This data shows that the LC-GEMS not only tracks closely with the C-sense on the scale of hours but also on the scale of minutes. Small fluctuations of $\mathrm{pCO}_{2}$ are observed with both sensors between 12:30 and 13:45. A net change of roughly $700 \mu$ atm $\mathrm{pCO}_{2}$ over the hour period is observed by both sensors. Note that this measurement is not believed to be sensor temperature drift because previous measurements have found minimal sensor drift of the C-sense over a 24 hour long measurement. Top: Each data point represents a 2 minute average reading from the C-sense and LC-GEMS sensors. Middle: The salinity and temperature readings from the RBRconerto CTD are plotted with dashed 30-point moving averages. Bottom: The depth of the sensors' inlets as measured by the RBRconcerto CTD are plotted in black along with a dashed 2-point moving average. The tide of Woodneck Beach (which is the inlet to Little Sippewissett Marsh, shown in Figure 2-17) is plotted in light blue and supplied from www.tidetablechart.com. The depth of the sensors is based on where the sensors could be positioned in the field at the time of deployment and does not always trend with relative tide level. 


\section{Interpretation of field measurement results}

For both the April 19th-20th and April 28th measurements, the marsh $\mathrm{pCO}_{2}$ content was highest in the early morning, and decreased to an observed low around mid-day before gradually increasing into the evening. This general trend matches the pattern expected for photosynthetic activity, with $\mathrm{CO}_{2}$ production from vegetation at night (thus increased $\mathrm{pCO}_{2}$ ) followed by increased $\mathrm{CO}_{2}$ consumption during the day (thus decreased $\mathrm{pCO}_{2}$ ). It is also evident that in both cases the minimum $\mathrm{pCO}_{2}$ coincided with the high tide and that as the tide went out in the afternoon the $\mathrm{pCO}_{2}$ generally trended upward. The increase of $\mathrm{pCO}_{2}$ with out-going tide matches the findings of previous studies of tidal marshes [97].

One key difference between the field measurements is that the morning peak $\mathrm{pCO}_{2}$, as measured by the LC-GEMS, was much lower on April 28th (2165 $\mu a t m)$ than on April 20th (4750 $\mu \mathrm{atm})$. This is likely justified by the fact that the full moon occurred on April 26th, shortly before the April 28th measurements. This means that the April 28th measurements occurred during a spring tide (the large tide cycle during full moon) and the April 19th-20th measurements occurred during a neap tide (the more moderate tide cycle when the moon is at a right angle to the sun) [98]. During spring tides the respiration of $\mathrm{CO}_{2}$ from tidal marshes is lower [98], which matches the lower measurement of peak $\mathrm{pCO}_{2}$ during the spring tide on April 28th.

Another difference between the measurements from April 28th and April 19th-20th is that April 28th shows an increase in $\mathrm{pCO}_{2}$ midday of approximately $700 \mu \mathrm{atm}$ over an hour (from 12:30 to 13:45, captured by both the LC-GEMS and C-sense in Figure 222) where as on April 20th, there is a very gradual afternoon increase of $200 \mu a t m$ over four hours (Figure 2-20). Firstly, this observation is believed not to be sensor temperature drift because previous measurements have found minimal sensor drift of the C-sense over a 24 hour long measurement. In this drift-assessment measurement, the C-sensor only drifted approximately 16 ppm over the course of 24 hours, a much slower rate of drift than the measurement in Figure 2-22. One primary reason for

this could be that the LC-GEMS was not run for longer that 5 minutes on April 20th 
and would not have picked up on these kinds of short-term trends at one constant location. Another key difference, as seen in the solar power data, is that April 28th had less intense sun exposure and was partly cloudy throughout the day, which could have impacted photosynthesis activity. Additionally, the tidal change was much larger on April 28th than on April 19th-20th (caused by the spring tide) and a total of 8 $\mathrm{cm}$ of depth was lost to the tide throughout the hour-long measurement (Figure 2-22 bottom plot). Tide (i.e. water depth) and ecosystem respiration have an inverse relationship because water level can impact $\mathrm{CO}_{2}$ diffusion as well as photosynthetic activity [98]. This means an out-going tide could potentially explain increased $\mathrm{CO}_{2}$ production. Additionally, the increase in water temperature could reflect an increase in soil temperature, which is positively correlated with $\mathrm{CO}_{2}$ from marshes [99]. It is important to note in this discussion that the $\mathrm{CO}_{2}$ flux from marshes is recognized as having a complex pattern with tide and daylight on daily time-scales [97] and has shown varied relationships with both [100].

\subsubsection{Discussion of Field Performance}

As shown in Figures 2-20 and 2-21, the LC-GEMS is capable of capturing the daily trends of dissolved $\mathrm{CO}_{2}$ content in the Little Sippewissett Marsh. Figure 2-23 plots the agreement between the LC-GEMS and C-sense measurement. The median percent difference between the LC-GEMS and C-sense reading was $19.9 \%$ on April 28th. Previous studies have compared the accuracy between different gas extraction and equilibration techniques for $\mathrm{pCO}_{2}$ measurement $[29,101,102]$ and they have reported percent differences among techniques of $9-15 \%$. This shows that the LC-GEMS system performs just slightly outside of the range of expected accuracy among current dissolved $\mathrm{CO}_{2}$ sensing techniques.

Thus, while the percent difference between the LC-GEMS and C-sense is indeed

a significant error for some applications where ppm level accuracy is needed, it is actually common for gas extraction measurement systems to disagree close to this order of magnitude. Thus, the LC-GEMS is a valid and promising technique for obtaining representative trends as well as preliminary data from field sites. 


\section{Measurement Agreement Scatter Plot of LC-GEMS and C-sense Data from Little Sippewissett Marsh Field Work}

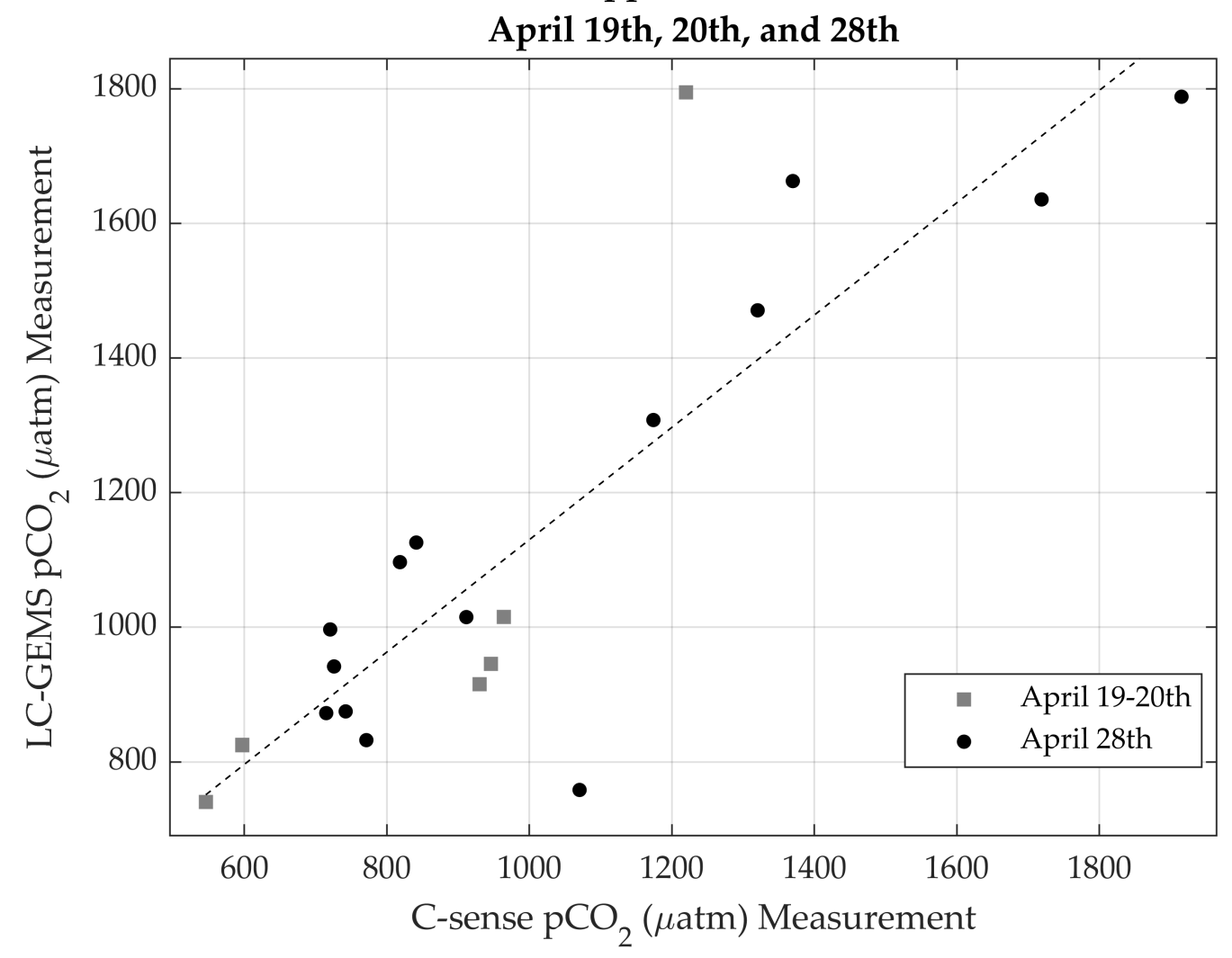

Figure 2-23: Comparison of the LC-GEMS and C-sense $\mathrm{pCO}_{2}$ readings for April 19th, 20th, and 28th field tests. Each data point is from Figures 2-20 and 2-21 excluding the points where the C-sense was railed-out. The median percent difference between the LC-GEMS and C-sense reading on April 28th was 19.9\% (grey squares), similar to the agreement previously found among other gas extraction systems [101]. The median percent difference between the LC-GEMS and C-sense reading on April 19th and 20 th was $21.3 \%$ (black circles). This discrepancy is attributed to the high water temperatures and future iterations on LC-GEMS calibration protocols are expected to improve this accuracy. 
The median percent difference on April 19th-20th between the LC-GEMS and C-sense was $21 \%$. This slightly larger difference is attributed to the high water temperatures (up to $22^{\circ} \mathrm{C}$ ) that were not covered in the lab calibration experiments. It is expected that future lab calibration that cover these high temperatures will build better multivariate relationships between sample water temperature and LC-GEMS reading to determine true $\mathrm{pCO}_{2}$ content.

Additionally, the results in Figure 2-22 reflect the LC-GEMS' capability for shortterm analysis. This plot shows that the LC-GEMS is not only capable of monitoring dissolved $\mathrm{CO}_{2}$ trends over hour long time scales, but also changes on the scale of minutes.

Beyond measurement accuracy, the field testing was also a good test for the LCGEMS robustness for field depoloyability. For the most part, the mesh water inlet filter was effective at preventing silt or debris from entering the LC-GEMS. However, during a couple trial runs, the filter became submerged in the mud and silty water was observed in the LC-GEMS. In these cases, the LC-GEMS $\mathrm{CO}_{2}$ reading was greatly increased. The increased $\mathrm{CO}_{2}$ reading is attributed to agitation of the sediment in the water flowing through the LC-GEMS extracting more $\mathrm{CO}_{2}$. Disturbing sediment re-mineralizes stored organic carbon into $\mathrm{CO}_{2}$ (which is the reason sea-bed dredging releases more $\mathrm{CO}_{2}$ to the atmosphere than aviation annually [103]). Simple clearing of the mesh filter by opening and removing any clogged debris would resolve the issue. Silty and muddy water does not damage the LC-GEMS because the water only flows through tubing; there are no delicate filter materials that are vulnerable to clogging or bio-fouling easily.

Data were successfully streamed to a laptop roughly 15 feet from the LC-GEMS for live data monitoring, though at times when the tide was highest the LC-GEMS was too far from the laptop to achieve a data connection.

\subsubsection{Conclusion}

The goal of a system like the LC-GEMS is to give research groups access to a low-cost and portable means to investigate dissolved gas trends and cycling. For some cases, 
the precision of the LC-GEMS would be sufficient for the research questions scientists have about a specific area. In other cases, the LC-GEMS may act as a good first pass to determine if an area is of interest for further high precision analysis with more expensive equipment.

As discussed in Section 2.6.4, the percent difference between the LC-GEMS and reference C-sense is on a similar order of magnitude compared to other current gas extraction techniques $[29,101]$ for certain temperature changes. Future improvements can be made to the calibration protocol for the LC-GEMS to achieve good measurement agreement for temperatures outside the range of $15-20{ }^{\circ} \mathrm{C}$ used to calibrate in this study. Additionally, future studies should evaluate the measurement drift associated with the LC-GEMS for assessing accuracy on longer duration deployments.

The LC-GEMS proved robust against environmental factors such as silt and organic debris, which is significant for gas extraction systems, seeing as the common membrane technologies are very vulnerable to clogging. Future work can address longterm durability against sediment and particulates by integrating water inlet filters that are more resistant against mud and silt clogging. Additionally, the LC-GEMS was designed to be hand portable and simple to use in the field. The LC-GEMS could easily be adapted for use on-board a surface vehicle. Future iterations of the LC-GEMS can adapt the system packaging to enable it to be left unattended for hour, day, or week long measurements at a single location. 


\section{Chapter 3}

\section{Planar nanophotonic structures for intensity based readout refractive index sensing applied to dissolved methane detection}

This thesis chapter was published in the Optical Society of America's Continuum Journal, Blevins et al. 2020 [104], and a reformatted version is reproduced here.

As introduced in Chapter 2, there is a need for low-cost and portable dissolved gas sensors that enable rich spatial and temporal data collection. Chapter 2 introduces a means to solve this problem using a rapid, low-cost, and portable gas extraction system with low-cost commercial gas sensors. This approach sticks with the convention of first extracting gas from the water phase and then analyzing the sample in the gas phase. Another path toward making more portable dissolved gas sensors would be to by-pass the extraction step and measure the dissolved gas in the water phase. There are reasons however this has not already happened. Water is very absorptive to light, 
so the traditional optical cavities of IR absorption sensors cannot work in water. The NDIR sensors used in Chapter 2 similarly cannot work if the optical channel was filled with water. This Chapter presents an approach for measuring dissolved gas in the water phase with a photonic approach. Photonics is the field which studies the optical properties of materials and light-matter interaction [105]. The applications of photonics are extremely broad, ranging from communication to sensing to computing [105]. Here, the design and simulation results for novel photonic dissolved methane sensors are presented.

\subsection{The Motivation for a Photonic Approach to Methane Sensing}

The global methane cycle describes the balance between the sources and sinks that make up the concentration in our atmosphere. While anthropogenic production of methane through agriculture and energy use is significant, natural sources like the ocean and Arctic are becoming increasingly important to quantify for understanding methane's role in climate change. As global temperatures rise, permafrost in the Arctic is thawing, stimulating increased release of methane [106]. The extent to which natural sources are contributing to increasing global methane levels is not well understood [107]. This motivates the need to better quantify methane release from water systems, especially in the Arctic, where significant methane outgassing events have recently been detected [108]. In Arctic near-surface waters, the methane concentration can range from as low as 3-10 $\mathrm{nM}$ (atmospheric equilibrium) to greater than $800 \mathrm{nM}$ during seasonal ice melt [18].

As described in Section 1.2.2, currently available state-of-the-art gas extraction and analysis techniques, which are costly and bulky, are impediments to research, as methane outgassing often occurs in remote locations, such as the Arctic Ocean or in rural lakes or rivers, that are difficult and often dangerous to access. The development of small, portable dissolved methane sensors, which are either handheld or deployable 
on underwater vehicles, would enable more widespread studies and measurements in waterways that are currently routinely inaccessible.

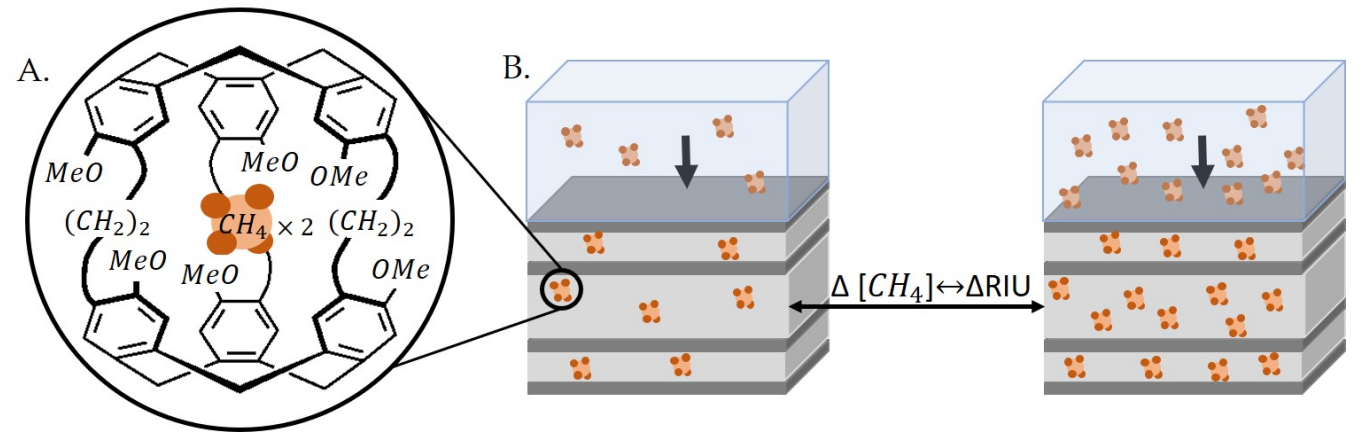

Figure 3-1: (A) A schematic of a cryptophane-A molecule hosting methane is shown. (B) Cryptophane-A doped layers of PDMS (light gray) traps methane while the undoped layers of PDMS (dark gray) allow the methane to permeate. As the concentration of methane increases in the surrounding environment, more methane is absorbed into the structure and the refractive index of the sensing layers increases.

Cryptophane-A is a molecule that selectively traps methane (Figure 3-1A) [109]. When cryptophane-A is mixed into a gas permeable polymer, e.g., polydimethylsiloxane (PDMS), the absorption of methane into the polymer changes its refractive index (RI) (Figure 3-1B). This change in RI can be monitored and used to measure methane concentration in the atmosphere or in aqueous solutions. A recipe for making cryptophane-A doped PDMS is outlined by Torino et al. (2017) [110]. CryptophaneA doped polymers have been used as sensing elements in optical sensors based on excitation of Surface Plasmon Resonances (SPR) to measure methane [110,111]. To the best of our knowledge, Boulart et al. (2013) presented the only cryptophaneA based sensor that has been deployed in the ocean for in situ dissolved methane measurements, based on a modified commercial Spreeta sensor platform [111,112]. Their SPR cryptophane-A device achieved a sensitivity to a lower limit of detection of $0.2 \mathrm{nM}$ dissolved methane [111]. However, the PDMS sensing layer peeled regularly from the SPR Spreeta chip in seawater, requiring regular replacement of the sensor layer and reflecting the need for a more robust configuration [112]. Torino et al.'s SPR sensor was implemented in a microfluidic chamber and characterized in the lab [110]. Other applications of cryptophane-A for methane sensing have re- 
quired solid-state laser sources and/or expensive spectroscopy equipment to make their measurements [113-116]. These past iterations of cryptophane-A based sensors demonstrate its potential for high sensitivity and selectivity in dissolved methane sensing. Cryptophane-A based sensors have been shown to be insensitive to common interfering gases such as $\mathrm{CO}_{2}, \mathrm{H}_{2}$ and $\mathrm{O}_{2}$ [115]. Sensitivities to bulk gas refractive index change can be accounted for with the use of a reference sensor and though Cryptophane-A is cross sensitive to xenon, radon, and chloromethanes, these gases are not a common concern in ocean and arctic dissolved gas measurements [114]. Despite the results of these prior works, these past implementations of cryptophaneA sensors reveal that more robust and simple approaches are needed to make them feasible for use in the field.

To address the above challenges, we design and compare the performance of several planar nanostructures as optical transduction elements containing one or more layers of cryptophane-A doped PDMS as the sensing layer(s). These structures include (1) a Bragg reflector with a defect layer, (2) a hybrid plasmonic-photonic multi-layer stack that supports a Tamm plasmon mode, and (3) a hybrid plasmonic-photonic stack that acts as a magnetic mirror, and can all be used in a simple intensity-based measurement scheme with low-cost light sources and detectors. The nanostructures are designed to support narrow-band resonances in the visible and near-infrared spectral range, whose spectral positions and angles of critical coupling to the light from external light sources vary with the changes in the RI of doped layers. Figure 3-1A illustrates how cryptophane-A doped layers capture dissolved methane and change RI, while undoped layers of PDMS allow methane to permeate.

Planar photonic and plasmonic nanostructures composed of multiple layers of dielectrics and metals offer thin (measuring from hundreds of nanometers to tens of micrometers), lightweight, and low-cost sensing elements with high surface area, which can be easily fabricated by high-throughput deposition techniques, such as sputtering, chemical vapor deposition, and spin coating $[117,118]$. Coupled with a small measurement system, photonic and plasmonic sensors can yield highly miniaturized and portable instruments, well suited for in situ measurements in water. These 
instruments could be made smaller and lower cost compared to currently available oceanographic dissolved gas sensors that use infrared spectroscopy or mass spectroscopy $[18,49]$. Here we compare and analyze three planar nanophotonic and plasmonic structures for dissolved methane sensing that can be coupled to a simple and low-cost intensity-based readout measurement scheme. The configurations here improve upon past cryptophane-A implementations by meeting these qualifications:

- Robust for use in freshwater and seawater: designed with materials that have previously been demonstrated to achieve a strong connection between layers via adhesion techniques (no peeling underwater), that do not degrade in water, and that do not significantly swell underwater or compress under surface water pressure

- Simple and low-cost to implement to create a portable instrument

- Designed for improved performance in a reflectance intensity based readout mode

\subsection{Methods}

In this section, the designs of three planar structures are presented and compared to an SPR Spreeta chip for use in dissolved methane sensing. The structures are designed for simplicity of fabrication, robustness, and optimized performance in a reflectance intensity readout mode.

\subsubsection{Reflectance Intensity based readout mode}

Although multiple configurations of miniaturized sensor chips have been proposed and explored, many of them require integration with tunable laser sources and/or spectrometers to measure the shift of the sensor optical modes spectrum in response to environmental changes [119]. Spectrometer-free biosensors have been created that weigh as little as 60 grams and are only $7.5 \mathrm{~cm}$ tall [119]. One option to avoid the 
need for a spectrometer is to measure the angular shift of resonant peaks with an arrayed photodetector [111]. This technique is used for structures with a strong angular shift response such as SPR sensors [111]. For structures with sharp and steep resonant peaks, another technique is to monitor reflectance intensity at a fixed incident angle and wavelength (Figure 3-2A) [119-122]. For example, Jahn et al. (2015) developed a handheld biosensor system based on measuring the optical transmission intensity change of a photonic crystal sensor binding to molecules [121]. This technique uses a narrow-band low-cost light emitting diode (LED) excitation source and a complementary metal-oxide semiconductor (CMOS) camera. Crossed polarization filters are used around the sensing cell for background signal removal. An optical reflectance intensity-based readout sensor provides the low-cost and portability advantages as well as allows the sensor face to be submerged in water rather than require its integration with a microfluidic chamber (Figure 3-2B).

A.

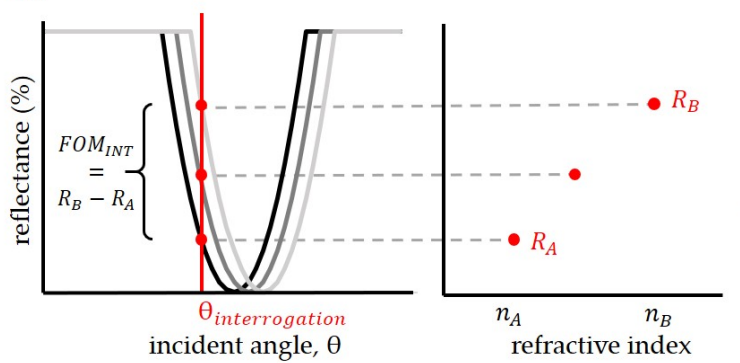

B.

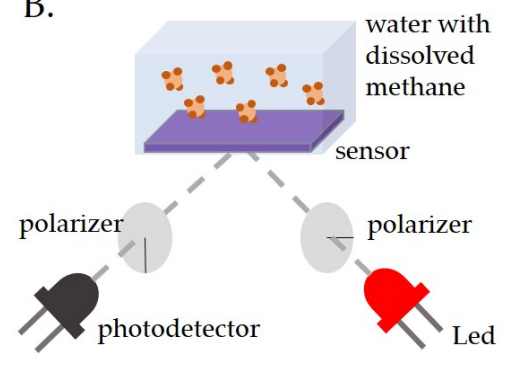

Figure 3-2: (A) Schematic illustrating the concept of the intensity-based (INT) readout of an RI sensing structure. The intensity at a single angle interrogation is monitored and translated to an RI measurement. The figure of merit $\left(F O M_{I N T}\right)$ of this measurement scheme is the total change in reflectance, RB-RA, for the RI range $n_{A}$ to $n_{B}$. (B) Schematic of a possible configuration of the sensor readout system. A narrowband LED, possibly integrated with spectral filters, acts as the excitation source for the sensor and the intensity of the reflected signal is measured with a photodetector. Crossed polarization filters allow measurement of spectral responses of two orthogonal polarizations separately and help to suppress background signals [120].

In an intensity-based readout system, the reflectance intensity is monitored at a single incident angle, the interrogation angle, for a fixed wavelength source [122]. As the RI of the sensing layer changes, the resonant peak shifts and the reflectance at the interrogation angle increases or decreases accordingly (Figure 3-2A) [122]. For a 
refractive index range of $n_{A}$ to $n_{B}$ the total reflectance intensity change is equal to RB-RA (Figure 3-2A). We define this value as the figure of merit (FOM) for intensity based (INT) sensors:

$$
F O M_{I N T}=R_{B}-R_{A}
$$

The best achievable $F O M_{I N T}$ for a defined RI range is $100 \%$, corresponding to the system response changing between that of a perfect absorber and a perfect reflector. A reflectance intensity based readout mode configuration for a dissolved methane sensing chip is illustrated in Figure 3-2B. Expanding this capability to environmental measurements will enable portable and low-cost in situ sensing.

\subsubsection{Design of planar nanophotonic structures for reflectance intensity based readout}

The planar structures presented in this paper were designed to produce sharp resonant spectral features in the visible to near-infrared wavelength range, 500-900 nm, that shift in response to changing the RI of the sensing layer(s), $n_{s}$. This wavelength range was selected to enable the use of low-cost light sources and detectors/cameras. The sharpness of the resonant peaks was engineered to measure the full range of RI expected for the methane sensor (as discussed in Section 3.2.1). The RI range used was $n_{s}=1.41198-1.41358$ RIU $\left(\Delta n_{s}=0.0016\right)$, corresponding to approximately $0 \mathrm{nM}$ to $300 \mathrm{nM}$ dissolved methane for cryptophane-A doped PDMS [111]. All presented structures were designed to have corresponding full width at half the maximum (FWHM) and angular sensitivity $(\% / \mathrm{RIU})$ to create the largest $F o M_{I N T}$ for the $1.41198-1.41358$ RIU range.

We chose the following four types of structures to be optimized for the dissolved methane sensing: (i) thin metal film mounted on a grass prism, which support a surface plasmon polariton mode, (ii) a Bragg reflector composed of dielectric materials with a single defect, which supports a trapped optical mode, (iii) a multi-layer metal-dielectric stack that supports a Tamm plasmon mode, and (iv) a multi-layer metal-dielectric stack that acts as a magnetic mirror. Some of these structures have 
been previously proposed for use as bio(chemical) sensors with an optical readout, and the surface plasmon resonance (SPR) sensors technology has been successfully commercialized for both refractive index sensing in solution and affinity-type biological sensor assays. However, with the exception of the SPR sensor, these platforms have not been yet optimized for use in intensity-readout optical sensors of dissolved methane, which requires precise calibration of the structure to simultaneously achieve high sensitivity and provide the required dynamic range corresponding to the expected range of methane concentration in terrestrial water sources. Furthermore, a planar metal-dielectric magnetic mirror design proposed in this manuscript has never been proposed before to the best of our knowledge. This new type of structure provides a simple yet ultra-sensitive platform for use in planar biochemical sensor platforms, beyond the dissolved methane sensor applications considered in this study. The following sections provide detailed comparative analysis the optimized designs of the four structures and compare them to each other under identical conditions with the goal to provide practical design guidelines for the development of new sensor platforms for compact methane sensors and monitors.

\subsubsection{Reference Surface Plasmon Resonance (SPR) sensor}

The SPR configuration used by Boulart et al. (2008) acts as the baseline configuration (Figure 3-3A), to which the other structures designed in this paper are compared. Their design consists of a $100 \mu m$ thick film of cryptophane-A doped PDMS adhered onto an SPR Spreeta chip. The Spreeta chip tracks the angular position of the structure's resonance peak (Figure 3-3B) with a diode array detector [123]. In SPR sensors, the excitation of a surface plasmon polariton mode on the metal-dielectric interface creates a strong localized electric field enhancement at the surface of the metal film, which evanescently extends into the sensing layer (Figure 3-3C). Coupling of the incident light to the surface plasmon mode is manifested as a resonant dip in the reflectance spectrum, whose spectral and angular position are sensitive to the RI changes in the sensing layer (Figure 3-3B). SPR configurations have been

used to measure dissolved methane with a cryptophane-A doped PDMS sensing layer 
$[111,112]$. Though sensitive to the RI change, the resonant peak for SPR sensors is relatively wide (Figure 3-3B), as metal is inherently lossy [124]. This makes SPR sensors better suited for use in a sensor configuration optimized for angular shift monitoring, rather than in sensors that use intensity-based readout for tracking small changes in $n_{s}$.

A.

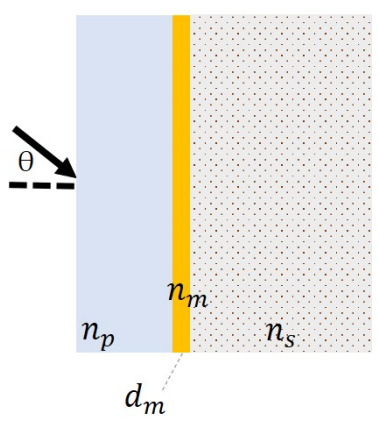

B.

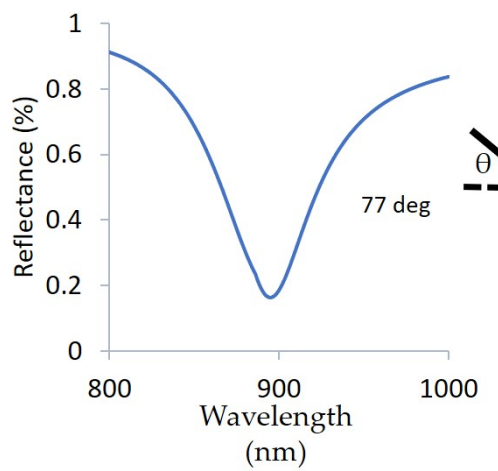

C.

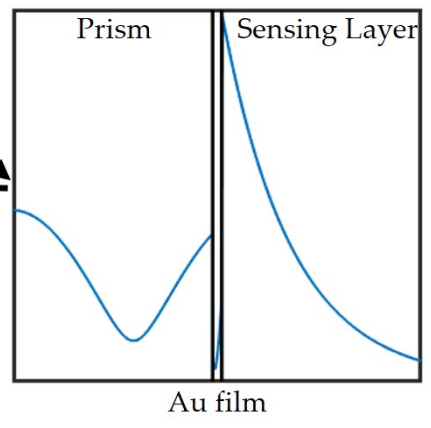

Figure 3-3: (A) The structure of an SPR RI sensor, based on a Spreeta chip. The excitation light passes through a prism of $n_{p}=1.5$ to a gold ( $\left.\mathrm{Au}\right)$ film $\left(d_{m}=50 \mathrm{~nm}, n_{m}\right.$ as defined by CRC Materials Science and Engineering Handbook [125]) on top of a cryptophane-A doped PDMS sensing layer $\left(n_{s}=1.41198\right.$ for $\left.0 \mathrm{nM} \mathrm{CH}_{4}, d_{s}=100 \mu \mathrm{m}\right)$. (B) The resonant dip in the reflectance spectrum of the structure at incident angle 77 degrees. Note that the x-axis is $200 \mathrm{~nm}$ in width while the reflectance spectra shown for the following structures have much narrower dips and are plotted over 10 $\mathrm{nm}$. (C) The relative p-polarization electric field distribution inside the structure at the resonant wavelength of $850 \mathrm{~nm}$. The surface plasmon polariton mode is excited at the interface between the Au film and the sensing layer, which produces a resonant dip in the structure's reflectance spectrum.

\subsubsection{Bragg reflector with a single defect layer (Bragg defect structure)}

A Bragg reflector with a central defect layer with the low index layers of the reflector structure all acting as sensing layers can provide an alternative sensor configuration, which offers higher sensitivity in the intensity readout mode. An example of a new sensor configuration is shown in Figure 3-4 a. All layers of this structure are designed to be composed of PDMS, with high-index layers composed of PDMS doped with $\mathrm{TiO}_{2}$ 
nanoparticles, and low-index layers of PDMS doped with cryptophane-A. Doping of PDMS with $\mathrm{TiO}_{2}$ nanoparticles increases the refractive index of the composite material without loss of optical transparency, and provides index contrast with the cryptophane-A necessary for the formation of the Bragg mirror. The thicknesses of the layers have been optimized to form a photonic band gap (PBG) centered around $700 \mathrm{~nm}$ wavelength. This structure would allow gas to permeate all layers of the Bragg reflector, passing through the $\mathrm{TiO}_{2}$-integrated layers and become trapped into the cryptophane-A layers (Figure 3-4A). The sharp resonant dip within the PBG of the Bragg reflector spectrum, as shown in Figure 3-4B, is a result of inserting a defect layer at the center of the Bragg reflector. This creates a defect mode, in which the electric field is amplified within that layer, present in both $\mathrm{s}$ and p polarizations (Figure 3-4C) [126].
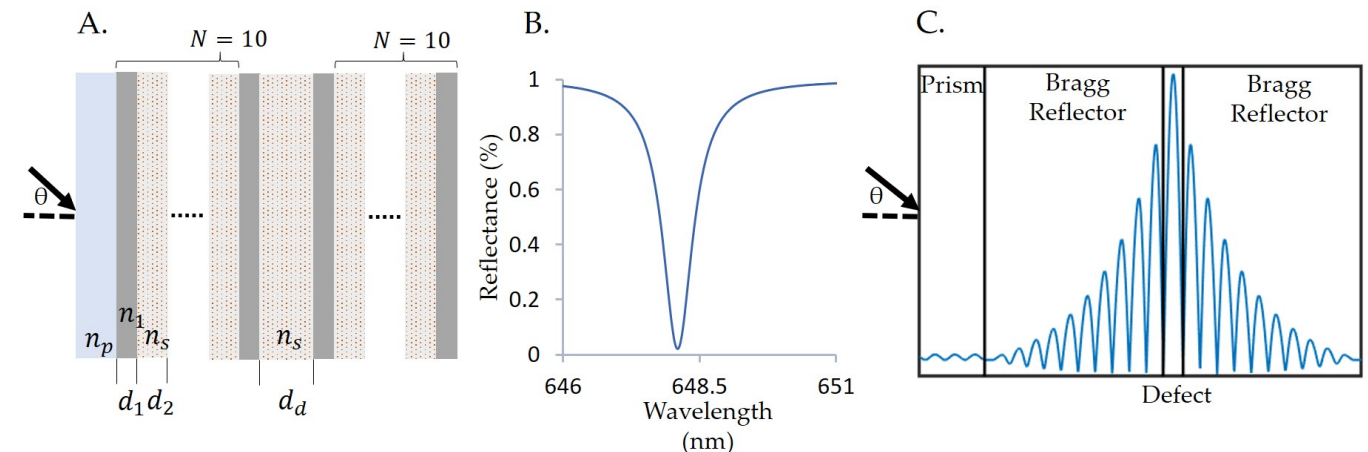

Figure 3-4: (A) The structure of the Bragg reflector with a central defect layer optimized for RI sensing. The excitation light passes through a prism of $n_{p}=1.4$. The Bragg reflector is formed from alternating layers of $\mathrm{TiO}_{2}$ - integrated PDMS $\left(n_{1}=1.68\right.$, $\left.d_{1}=119 \mathrm{~nm}\right)$ and cryptophane-A doped PDMS $\left(n_{s}=1.41198\right.$ for $0 \mathrm{nM} \mathrm{CH}_{4}, d_{2}=140$ $\mathrm{nm})$. The defect layer of cryptophane-A doped PDMS has width $d_{d}=300 \mathrm{~nm}$. (B) The resonant dip in the s-polarization reflectance spectrum of the structure at an incident angle of 40 degrees. (C) The s-polarization electric field of the structure at resonant wavelength $645 \mathrm{~nm}$, which corresponds to the excitation of the localized defect mode within the center layer.

The RI of the $\mathrm{TiO}_{2}$-integrated PDMS can be tuned through the fabrication process and can exceed 1.7 RIU [127,128]. Here, we use an RI value of $1.68 \mathrm{RIU}$ for $\mathrm{TiO}_{2}$ integrated PDMS. The sharpness of the resonant feature can be controlled by changing the number of periods in the Bragg reflectors. In this paper, the number of periods 
was chosen to maximize the total percent change of reflectance for the defined RIU range (0.0016 RIU). More periods would support a smaller RIU range, while fewer periods would support a larger RIU range. The spectral position of the resonant peak has been shifted by tuning the center wavelength of the PBG of the Bragg reflectors and the width of the defect. Polymer-based Bragg mirrors can be fabricated via conventional polymer film-making processes such as dip-coating from solution or meltextrusion. Multi-layer structures produced by these techniques have been shown to be amenable to large-scale manufacturing and can incorporate a variety of polymers with and without nano-dopants [129, 130].

\subsubsection{Multi-layer stack that supports Tamm plasmon mode (Tamm structure)}

This structure consists of a dielectric $\mathrm{SiO}_{2} / \mathrm{SiC}$ Bragg reflector with a thin $\mathrm{Au}$ film on one side and the polymer sensing layer on the other side (Figure 3-5A). This structure supports a Tamm plasmon mode, which is a hybrid mode localized at the interface between a metal film and a Bragg reflector [131-135]. Excitation of the Tamm plasmon mode is manifested as the narrow resonant dip in the reflectance spectrum of the structure, which shifts with refractive index change of the sensing layer below (Figure 3-5B). The electric field profile of the structure is shown in Figure 3-5C. Unlike SPP resonances, which can only be excited on metal surfaces with the p-polarized light, Tamm plasmon modes exist for both $\mathrm{p}$ and s orthogonal polarizations, and their spectral positions in the visible and infrared wavelength range of the structure (Figure 3-5B) can be tuned by varying the thicknesses of metal and dielectric layers as well as the excitation angle [134]. The sharp resonant peaks in the reflectance spectrum of this structure make it a good candidate for tracking small changes of $n_{s}$ via the intensity-based readout.

The sharpness of the Tamm plasmon resonant feature can be tuned by increasing the number of periods of the Bragg reflector. In our design, the number of periods was chosen to maximize the total percent change of reflectance for the defined RIU range 
(0.0016 RIU). More periods would support a smaller RIU range while fewer periods would support a larger RIU range. The spectral position of the resonant feature has been tuned optimizing the thicknesses of the dielectric layers comprising the Bragg reflector, and the number of periods.
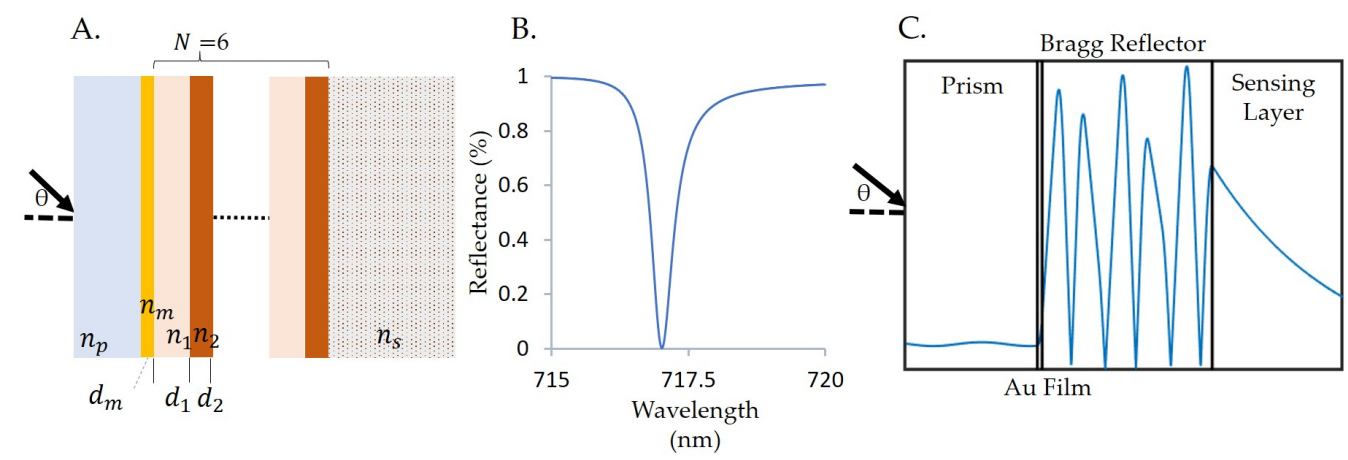

Figure 3-5: (A) The Tamm plasmon structure for RI sensing. The excitation light passes through a prism of $n_{p}=1.5$. A thin film of $\mathrm{Au}\left(d_{m}=40 \mathrm{~nm}, n_{m}\right.$ defined by [125]) covers a 6 period Bragg reflector with alternating layers of $\mathrm{SiO}_{2}\left(n_{1}=1.45, d_{1}=103.5\right.$ $\mathrm{nm})$ and $\mathrm{SiC}\left(n_{2}=2.6, d_{2}=57.7 \mathrm{~nm}\right)$. The sensing layer of cryptophane-A doped PDMS completes the structure $\left(n_{s}=1.41198\right.$ for $\left.0 \mathrm{nM} \mathrm{CH}_{4}, d_{s}=140 \mathrm{~nm}\right)$. (B) The resonant dip in the s-polarization reflectance spectrum of the structure at an incident angle of 70.73 degrees. (C) The s-polarization electric field of the structure at resonant wavelength $827.94 \mathrm{~nm}$. The evanescent field extends into the sensing layer.

\subsubsection{Hybrid multi-layer stack that acts as a magnetic mirror (Magnetic Mirror)}

In this structure, the sensing layer lies atop a thin Au film absorber coated on the surface of a planar photonic crystal magnetic mirror. The magnetic mirror is formed as a 16 period $\mathrm{SiO}_{2} / \mathrm{SiC}$ Bragg stack with a standard Au electric mirror back reflector (Figure 3-6A). This structure acts as a magnetic mirror at select frequencies by preserving the phase of the reflected wave and creating the constructive interference of the incident and reflected wave at its top surface [136-138]. This constructive interference condition promotes efficient narrow band absorption in the thin Au film at multiple tunable-by-design wavelengths in the visible spectral range (Figure 3-6B). The electric field at the Au-polymer interface is amplified, and the evanescent mode 
extends into the sensing layer (Figure 3-6C). The field excitation and the resulting light absorptance in the Au film creates sharp resonant features in the reflectance spectra for both $\mathrm{s}$ and $\mathrm{p}$ polarizations, whose positions are sensitive to the value of $n_{s}$.

The sharpness of the resonant feature, as well as the number of these features in the optical spectrum, can be tuned by increasing the number of periods of the Bragg reflector. In our design, the number of periods was chosen to maximize the total percent change of reflectance for the defined RIU range (0.0016 RIU). More periods would support a smaller RIU range while fewer periods would support a larger RIU range. The spectral position of the resonant feature has been tuned by changing the thicknesses of the high and low index layers comprising the Bragg reflector and the number of periods.
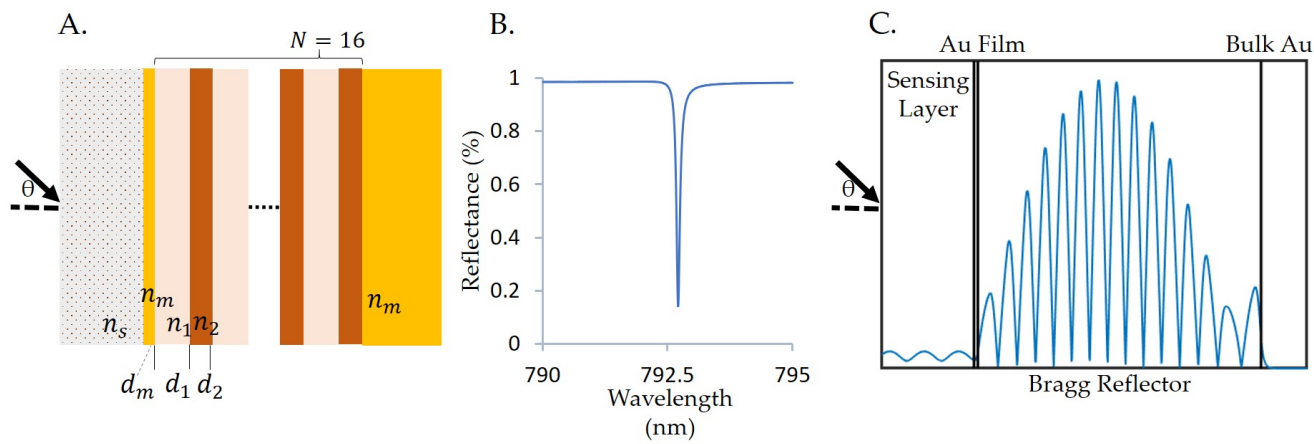

Figure 3-6: (A) The magnetic mirror structure for RI sensing. The excitation light passes through the PDMS sensing layer $\left(n_{s}=1.41198\right.$ for $\left.0 \mathrm{nM} \mathrm{CH}_{4}, d_{s}=1 \mu m\right)$. A thin film of $\mathrm{Au}\left(d_{m}=40 \mathrm{~nm}, n_{m}\right.$ defined by [125]) covers a 16 period Bragg reflector formed from alternating layers of $\mathrm{SiO}_{2}\left(n_{1}=1.45, d_{1}=125 \mathrm{~nm}\right)$ and $\mathrm{SiC}\left(n_{2}=2.6, d_{2}=65 \mathrm{~nm}\right)$. A layer of bulk Au completes the structure. (B) A resonant dip in the s-polarization reflectance spectrum of the structure at an incident angle of 41 degrees. (C) The relative s-polarization electric field of the structure at the resonant wavelength of 791 $\mathrm{nm}$. The field is amplified around the thin Au film coating interfacing the sensing layer, and the evanescent mode extends into the sensing layer.

\subsubsection{Simulation Methodology}

The planar structures in this paper were simulated using the Lumerical STACK photonic simulation software package, which uses the analytic transfer matrix method to 
model the optical behavior of planar multilayer stacks [139]. The spectral response for incident angles in the range between 0 and 90 degrees and for wavelengths in the 500-900 nm range was simulated for each structure. The STACK simulations used the structure parameters outlined in Sections 2.3-2.6, and all materials besides Au were approximated as lossless in the 500-900 nm range (no imaginary part of the dielectric constant). The parameters of Au were defined by the CRC Materials Science and Engineering Handbook [125]. The RI of $\mathrm{SiO}_{2}$ was defined by Malitson et al. (1965) and the RI of $\mathrm{SiC}$ was defined by Singh et al. (1971) [140,141].

\subsection{Results and Discussion}

\subsubsection{Comparable performance of the planar sensor structures}

The spectral response of the four sensor configurations for incident angles of 0-90 degrees and wavelengths in the 500-900 nm range are shown in Figure 3-7. Here, the calculations are performed assuming $0 \mathrm{nM}$ of dissolved methane $\left(n_{s}=1.41198 \mathrm{RIU}\right)$, corresponding to a blank measurement, when methane is absent from seawater. The sharpest resonant dips were observed in the s-polarization for each structure (except the SPR sensor), but sharp dips are also present in the p-polarized reflectance spectra of the three improved sensors.

It should also be noted that, unlike the reference SPR sensor, the new sensor structures exhibit multiple resonant modes in their optical spectra. They also provide efficient coupling to light incident at normal and oblique angles, differently from the SPR sensor, which needs to be illuminated at a large oblique angle to excite a surface plasmon polariton mode. This offers flexibility of designing sensors to operate at multiple frequencies within the visible spectral range, and at different incident angles, potentially simplifying sensor designs and reducing their physical footprints. The calculated angular shift of each structure in response to a change in the RI of the sensing layer(s) is shown in Figure 3-8. The SPR structure exhibits the largest angular shift in resonant dip position. The other structures exhibit smaller angular 


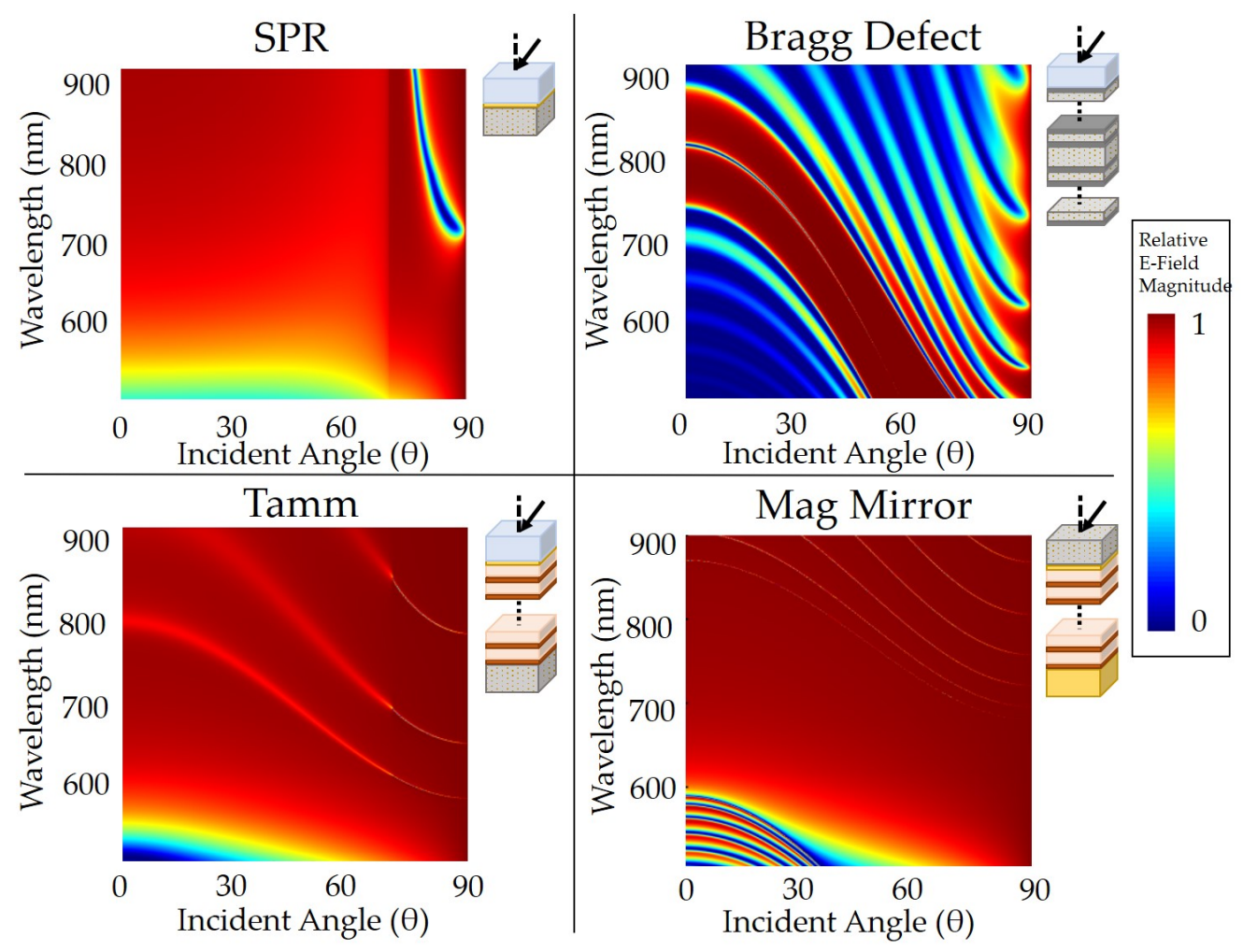

Figure 3-7: The reflectance spectra for the three improved sensor configurations (spolarization) and for the reference SPR sensor (p-polarization). Each of the three new structures exhibits resonant modes in both polarizations, with s-polarization giving the sharpest resonant peaks. SPR only supports a resonant mode in p-polarization. The blue-colored areas correspond to the resonant reflectance dips, whose dependence on the angle of incidence and wavelengths are unique for each structure. Note that the linewidth of the resonant mode in the SPR configuration is much larger than for the other structures.

shifts of their resonant features; however, the dips in their reflectance spectra are much sharper and are better suited to provide an optical readout mechanism for the reflectance intensity monitoring to trace a small RI change. Figure 3-9A plots the intensity vs. $\Delta n_{s}$ values obtained at a fixed wavelength and incident angle for each structure (indicated with the red dashed lines in Figure 3-8). By designing the structures for the RI range for 0-300 $\mathrm{nM}$ dissolved methane, a reflection intensity change of up to $76 \%$ is predicted for each structure (Table 1). Figure 3-9A shows the intensity change magnitude for specific wavelength and incident angle pairs, optimized separately for each structure. Our calculations predict that each structure outperforms 
the SPR configuration significantly by this metric. Figure 3-9B shows the angular shift of each structure; and our data show that the SPR sensor outperforms every other structure by this metric. However, the angular shift monitoring sensor operation requires integration of multiple detectors into an array for the signal readout, increasing the sensor cost and complexity.

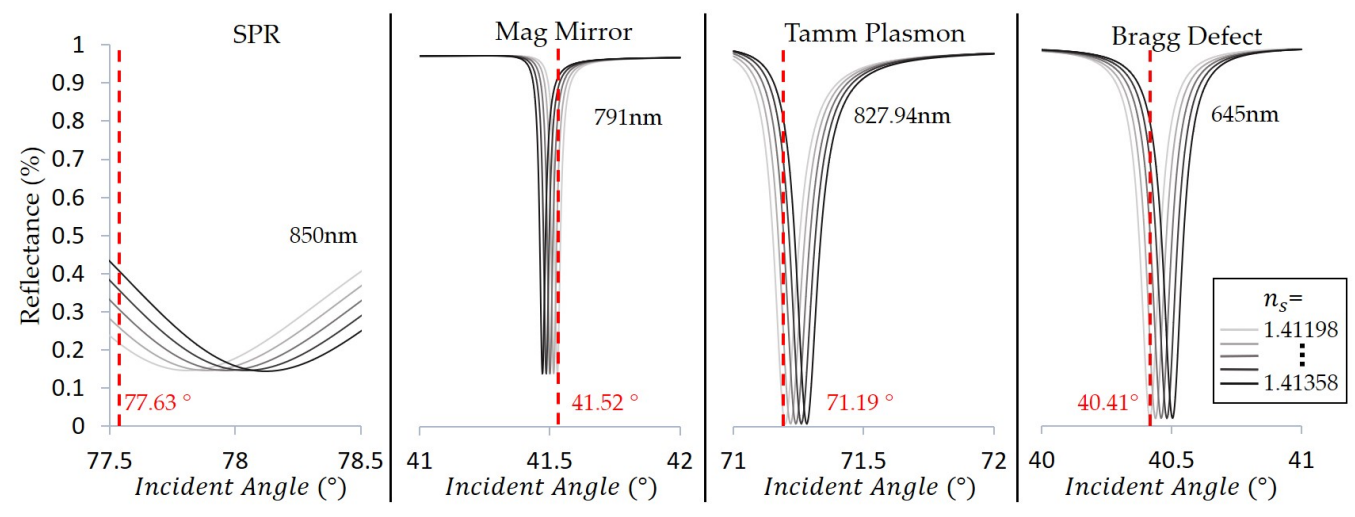

Figure 3-8: Angular shift of the resonant dip for each sensing structure for RI value changing in steps of 0.0004 RIU. The red dashed line shows the interrogation angle at which each configuration would be monitored to in the intensity-based readout implementation. The SPR configuration exhibits the resonant dip and the smallest change in intensity at the fixed wavelength. Resonant dip is shown for p-polarization for the SPR structure and for s-polarization for all the other structures.

Table 3.1 summarizes the characteristics of different sensor structures, and the figure of merits [142-144] for both the angular resonant mode shift readout and the reflectance intensity readout. The data in Figure 3-9 and Table 3.1 show that each of the nanostructures demonstrated at least a $76 \%$ change in reflectance intensity for the defined RIU range in simulation as compared to an $8.77 \%$ change for SPR. Larger values of $F O M_{I N T}$ are preferable for reflectance intensity readout sensing applications. The calculations show that each evaluated structure is expected to demonstrate larger $F_{O} M_{I N T}$ values than the SPR configuration, with the magnetic mirror and Bragg defect structures exhibiting the best performance (Table 3.1). The large FoM $M_{I N T}$ for each of these configurations resulted from both high sensitivity to the refractive index change (i.e., the large spectral shifts of the resonant modes) and narrow mode linewidths, and showed an excellent potential for intensity-based readout setups. For the angular resonant mode shift readout (ANG), the angular figure of 


\begin{tabular}{|c|c|c|c|c|c|c|c|}
\hline \multirow[b]{2}{*}{ Structure* } & \multicolumn{5}{|c|}{$\begin{array}{c}\text { Angular Resonant Mode } \\
\text { Shift Response }\end{array}$} & \multicolumn{2}{|c|}{$\begin{array}{c}\text { Reflection } \\
\text { Intensity Response }\end{array}$} \\
\hline & $\begin{array}{l}\text { Resonance } \\
\text { Wavelength } \\
\text { (nm) }\end{array}$ & $\begin{array}{l}\text { Sensitivity } \\
(\% / \mathrm{RIU})\end{array}$ & $\begin{array}{l}\text { FWHM } \\
\left({ }^{\circ}\right)\end{array}$ & $\begin{array}{c}\text { Quality } \\
\text { Factor } \\
\text { (Q) }\end{array}$ & $\begin{array}{l}\mathrm{FOM}_{A N G} \\
\left(\mathrm{RIU}^{-1}\right)\end{array}$ & $\begin{array}{c}\theta_{\text {critical }} \\
\left({ }^{\circ}\right)\end{array}$ & $\begin{array}{c}\mathrm{FOM}_{I N T} \\
= \\
R_{B}-R_{A} \\
\quad \%)^{* *}\end{array}$ \\
\hline SPR & 850 & 200.19 & 1.34 & 12.7 & 149.3 & 77.63 & 8.77 \\
\hline Tamm Plasmon & 827.94 & 51.32 & 0.1031 & 1096 & 487.7 & 71.91 & 76.78 \\
\hline Bragg Defect & 645 & 54.44 & 0.0821 & 957 & 663.1 & 40.41 & 78.36 \\
\hline Mag Mirror & 791 & 35.63 & 0.019 & 9218 & 1287 & 41.52 & 78.29 \\
\hline
\end{tabular}

Table 3.1: The performance metrics for each RI sensing structure. *The performance metrics are calculated for resonant modes in p-polarization for the SPR structure and in s-polarization for the other structures. These metrics correspond to the resonant mode responses shown in Figure 3-8. **As defined by the change in reflection intensity percent for the $R I U$ range the structures were designed for, i.e., for $\Delta n_{s}=0.0016 R I U$, corresponding to 0 nM to $300 \mathrm{nM}$ dissolved methane.

merit $\left(F o M_{A N G}\right)$ has also been calculated for each structure. This figure of merit is defined as the ratio of sensitivity (angular shift per change in RI) to FWHM. Larger values of $F o M_{A N G}$ are preferable for angular resonant mode shift sensing applications. Each evaluated structure exhibited higher $F_{O} M_{A N G}$ values than the reference SPR configuration, with the magnetic mirror offering the best performance on this metric (Table 3.1). This shows that despite their smaller sensitivities, the sharp peaks of the evaluated structures enable large $F o M_{A N G}$. The large $F o M_{A N G}$ for each of these configurations shows their potential for use in angular resonant mode shift readout setups.

Of all the planar sensor configurations considered above, the sensors based on the use of magnetic mirror and the Bragg reflector with a defect layer emerged as the most promising designs for dissolved methane sensing in the reflectance intensity based readout mode. They exhibit a large figure of merit $F o M_{I N T}$, and can be easily optimized for performance at a preferred wavelength and angle of incidence by tuning parameters of the structures. These designs are simple and low-cost to manufacture. Implementation of these structures with a simple intensity-based readout system would produce a low-cost and highly portable in situ instrument for dissolved methane sensing. In the following sections, we discuss a possible approach to further enhance the sensor performance by interrogating it with both p- and s-polarized light, 

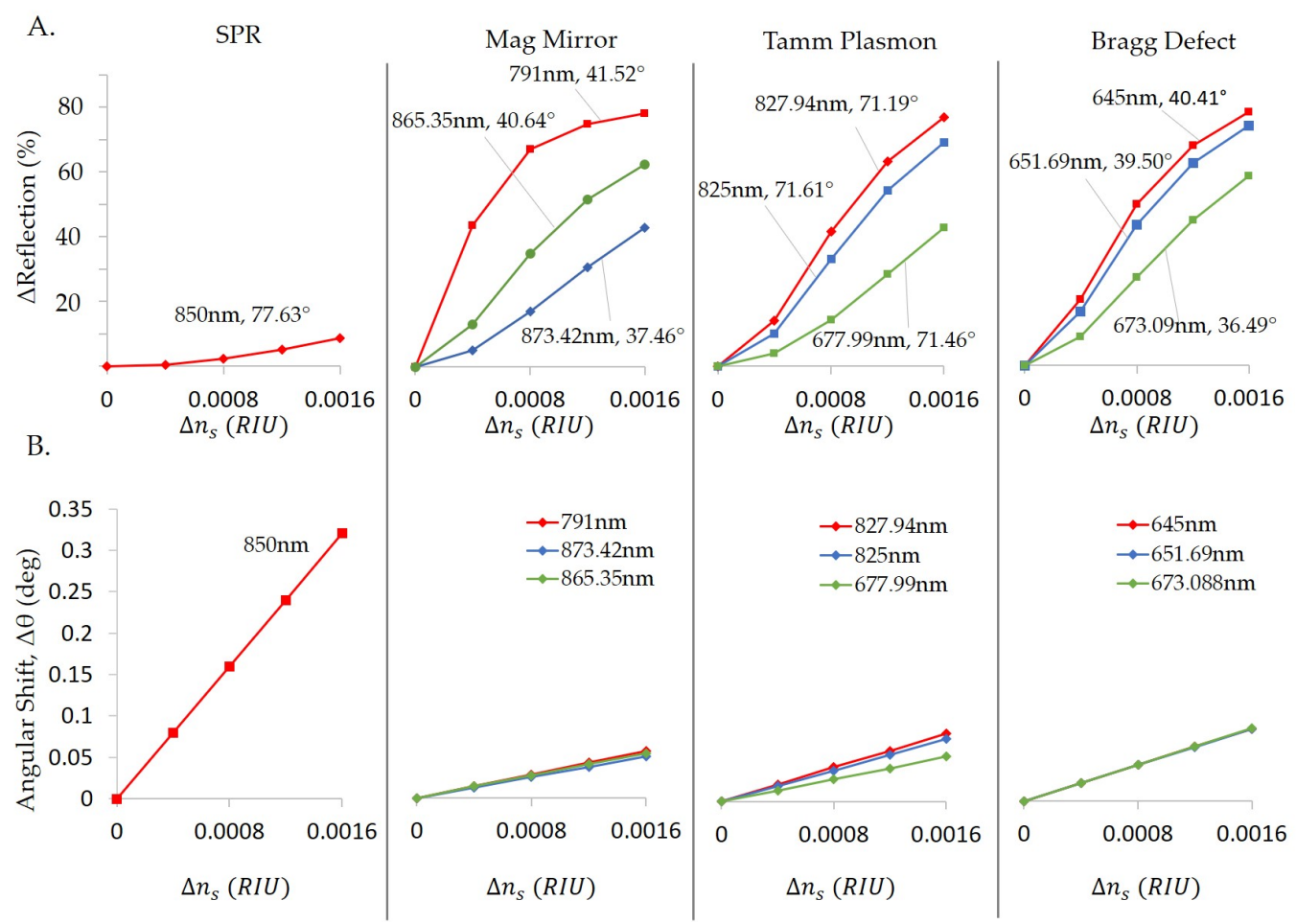

Figure 3-9: (A) Intensity based readout of each sensing structure. The intensity readout is plotted for multiple wavelengths and incident angle pairs. The red lines correspond to the data shown in Figure 8. Each of the structures is predicted to exhibit a larger response to the change in $n_{s}$ than the reference SPR configuration. Linearity of the response is tuned by optimizing the number of periods in each structure as well as by the selection of operational wavelength and the incident angle. (B) Angular shift of the reflectance resonant dip for each sensing structure. The shift is plotted for multiple wavelengths. The SPR configuration is predicted to exhibit the largest response.

and assess the robustness of the designed sensor structures for use in seawater.

\subsubsection{Measuring both polarizations for sensitivity enhance- ment}

The sensitivity of each of the proposed planar sensing structures can be enhanced by measuring the reflectance of both $\mathrm{s}$ and p-polarizations ( $R_{s}$ and $R_{p}$, respectively) and tracking the change of the value of the first ellipsometric angle, defined here as $\psi=\operatorname{atan}\left(\frac{R_{s}}{R_{p}}\right)$, in response to the RI changes in the sensing layer. No ellipsometer is required for these intensity-only measurements, instead simply both polarizations 
are monitored, and the ratio of the reflectances is calculated in the data processing steps. We have previously demonstrated that if a structure is designed to exhibit partially-overlapping resonant peaks/dips in both s and p-polarizations, this process enables enhanced sensitivity of detection $[133,134]$.

A.

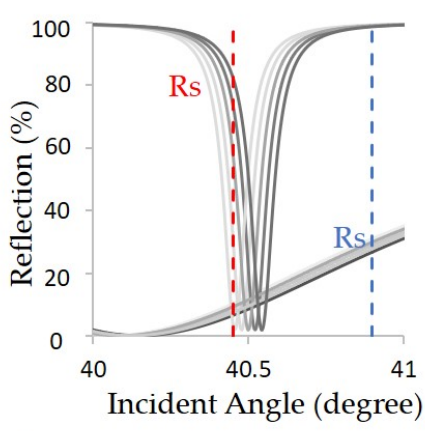

D.

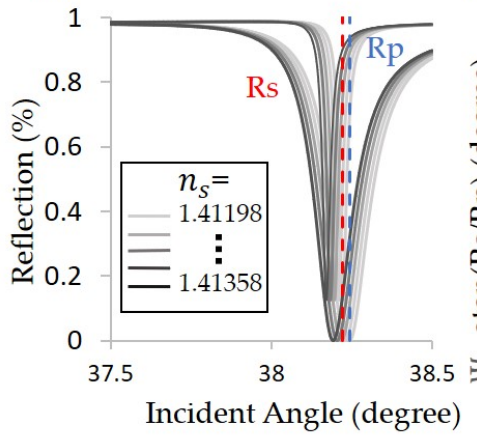

B.

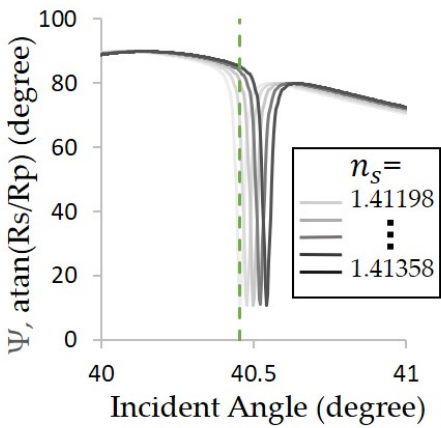

E.

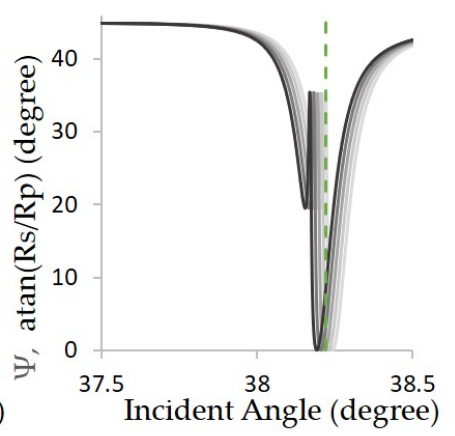

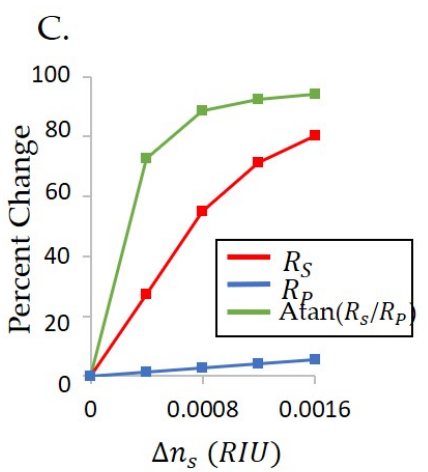

F.

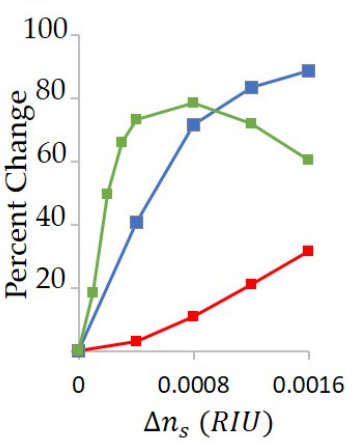

Figure 3-10: (A) The s and p-polarization reflectance of the Bragg Defect structure for a $645 \mathrm{~nm}$ excitation wavelength and the RIU range 1.41198 to 1.41358 of the sensing layer. The red and blue dotted lines show interrogation angles for s- and p-polarized light, respectively (B) The calculated s and p-polarization ratio is shown in the form $\psi=\operatorname{atan}\left(\frac{R_{s}}{R_{p}}\right)$. The green dotted lines show the interrogation angle. (C) The percent change of $\mathrm{s}$ and p-polarization reflectances $\left(\Delta R_{(s / p)}\right.$ divided by reflectance dip height) and $\operatorname{atan}\left(\frac{R_{s}}{R_{p}}\right)$ value as a function of the RI value change. (d-f) The same data as in (a-c), but for the magnetic mirror structure at a $801.57 \mathrm{~nm}$ excitation wavelength.

Figure 3-10 summarizes the performance of the two most promising structures for implementation in methane sensing - Bragg defect and magnetic mirror - but this technique can be expanded to improve the performance of any sensor structures exhibiting resonant features in both p- and s-polarized optical spectra. Our calculations predict that for the Bragg defect structure, the ellipsometric angle will exhibit a significant $72.8 \%$ change for $\Delta n_{s}=0.00004$ RIU, compared to a $27.3 \%$ and $1.3 \%$ change in the 
reflectance for the s and p-polarizations, respectively. This shows that combining the measurement of both polarizations can more than double the sensitivity of the sensor to small RI changes of the sensing layers. In turn, for the magnetic mirror structure, the predicted ellipsometric angle change is $73.2 \%$ for $\Delta n_{s}=0.00004$ RIU, compared to a $3.2 \%$ and $35.5 \%$ change in the reflectance for the s and p-polarizations, respectively. The sensitivity of this measurement can be defined as $S_{E}=\Delta \psi / \Delta n_{s}[134]$ and equals 55.0 $/ R I U$ for the Bragg defect structure and $31.9^{\circ} / R I U$ for the magnetic mirror structure. Finally, the low FWHM of the resonant features in Figs. 3-10B and e translates into improved $F o M_{A N G}$ of $1523 R I U^{-1}$ for the Bragg Defect (as compared to $663.1 R I U^{-1}$ for s-polarization reflectance) and $1385 R I U^{-1}$ for the magnetic Mirror (as compared to $1287.0 R I U^{-1}$ for the s-polarization reflectance).

\subsubsection{Discussion of chip robustness in seawater}

It is important to consider the potential swelling of polymer layers in nanostructures immersed in seawater, especially when the use of multiple layers is proposed. PDMS has a very low swelling ratio in water and is widely used in microfluidic devices without swelling [145]. Thus, it can be reasonably expected that nanostructures containing PDMS layers immersed in water would similarly not swell. Future experimental work can help to determine if any swelling occurs, which would further increase the spectral changes and might improve the sensitivity of the proposed structures as optical transducers for methane sensing [146]. Pressure is a similarly important factor to consider for underwater sensing. PDMS thin films have low compressibility and are in fact used in capacitive pressure sensors for this reason [147]. Under large hydrostatic loads however, PDMS membranes can compress and as a result become less permeable to gases [148]. However, thin films of PDMS of 500-3,000 nm thickness, like the structures presented here, have shown decreased compressibility compared to bulk PDMS $[148,149]$. The effect of hydrostatic pressure on the spectral response (from the changes in both layer thickness and permeation rate of gas) will need to be characterized. Thus, it can be expected that PDMS-based nanostructure sensors can be applied to both surface and subsea water measurements through experimental 
calibration.

The effects of temperature on such chips must also be considered. Temperature change in cryptophane-A doped polymers can cause phase drift, impacting the resonance condition of the chip and thus the output signal [114]. This can be address by stabilizing the temperature with a temperature controller, normalizing your output against a reference sensor, or through a combination of both techniques.

Another important factor that may negatively affect the sensor performance, is structural stability, which may be compromised by the long periods of exposure to water. For example, Boulart et al. (2013) attached the sensing layer of PDMS to a commercially-available Spreeta SPR chip; however, the PDMS layer often peeled off in seawater [112]. Adding a thin film of Chromium (Cr) between the Au and PDMS layers would provide a more robust adhesion of the polymer, but it would increase absorption losses, further broadening the SPR linewidth and thus degrading sensor performance [150].

In turn, the proposed Bragg defect structure can be fabricated by depositing alternating layers of cryptophane- $\mathrm{A}$ and $\mathrm{TiO}_{2}$-integrated PDMS via spin coating [127, 128]. Dalod et al. (2017) presented a method for fabricating PDMS Bragg reflectors with $\mathrm{TiO}_{2}$-integrated PDMS [127]. They constructed a 3-inch diameter chip, which demonstrated strong adhesion between layers and little dependence on temperature change [127]. This fabrication approach can be directly applied to the Bragg defect design. This shows the structure's potential for being robust for seawater measurements. This structure is also engineered for large surface area contact with water, enabling faster permeation of methane through the layers. The total thickness of the Bragg defect structure is less than $7 \mu \mathrm{m}$. Boulart et. al (2008) used a 100 $\mu m$ PDMS sensing layer in their SPR configuration resulting in a response time of 1 minute [111]. It can be reasonably expected that the structure proposed here could achieve a faster response time due to the reduced thickness.

The Tamm structure is also expected to be robust against long-term exposure to seawater. It has been previously shown that a strong bond can be formed between PDMS layers and oxidized silica with a primer called TMSM [151]. This primer 
forms a covalent bond between the layers, meaning manual peeling of the layers is not possible [151]. This addresses the need for a robust adhesive connection between the sensing layer and the rest of the nanostructure. Additionally, the sensing layer is designed to be immersed in water, while the rest of the structure interfaces with the measurement setup. This allows for large surface area contact.

Unlike the other structures, the magnetic mirror sensor configuration has been designed to have the sensing layer is on the side of the excitation source. This is unfavorable for in situ methane sensing in seawater; it would require the methane to enter from the sides of the structure (which have a very small surface area) or for the incident beam to pass through water. While this structure shows a high promise as a RI sensor for intensity-based readout, it could be better suited for a microfluidic-cellintegrated sensor implementation rather than in-situ seawater sensing. Additionally, this structure requires a connection between Au and PDMS films, which is weak, as found by Boulart et al. [112]. However, the high figures of merit exhibited by this sensor structure make in an interesting candidate for engineering planar sensors for high-sensitivity detection of very small RI changes.

Finally, the use of the Bragg defect structure in a practical low-cost and compact device configuration incorporating a light emitting diode (LED), a polarizer, and a narrowband filter is illustrated in Figure 3-11A. In this case, the intensity of reflected light is the product of the reflection coefficient and the filtered emission intensity at a specific wavelength (Figure 3-11B) [120,152]. Figure 3-11 shows the expected change in integrated intensity readout of the system as the refractive index of the sensing layers, $n_{s}$, increases with the increasing $\mathrm{CH}_{4}$ concentration.

\subsubsection{Conclusion}

To address the need for small, portable methane sensors that are capable of in situ measurements in aqueous environments, this paper presents the design of a dissolved methane sensor system that is robust, low-cost and simple to manufacture and only requires a small, low-cost measurement setup. The main goal of this manuscript has been to find and optimize a simple planar structure with the best performance for the 


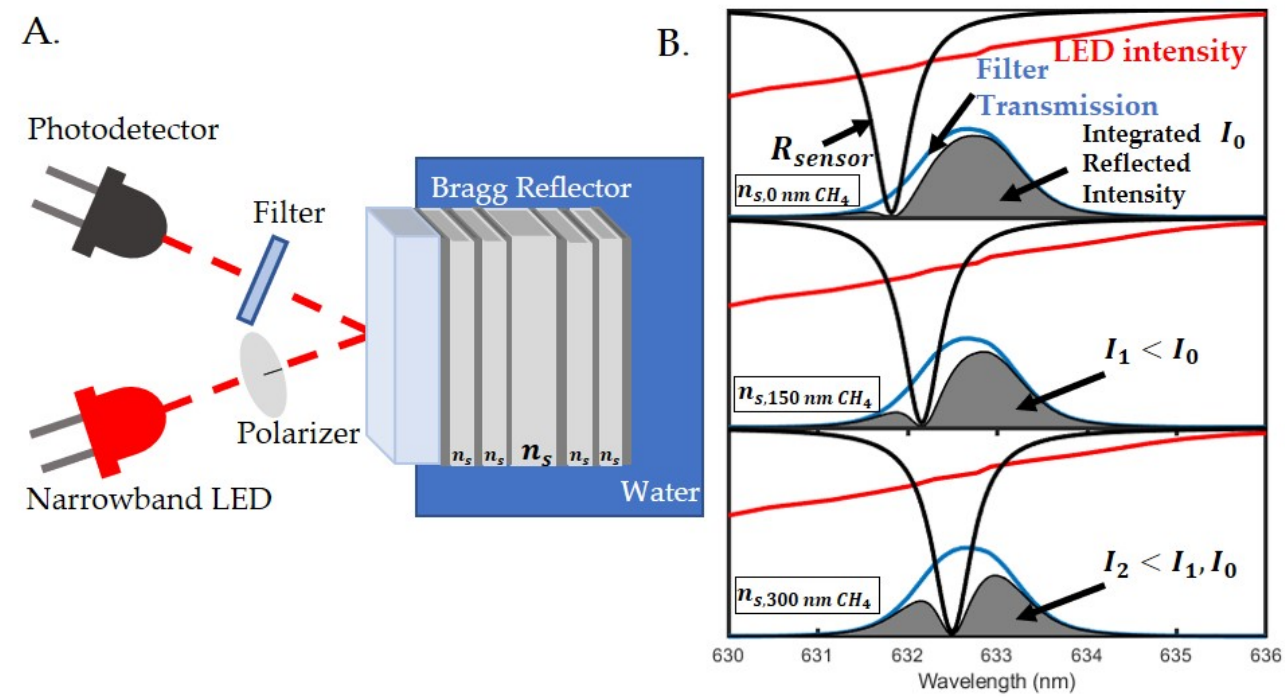

Figure 3-11: (A) Schematic of a reflectance intensity-based readout implementation of the Bragg defect structure. (B) The expected integrated reflectance intensity measurement of the Bragg defect structure shown in (A). The narrowband LED spectrum is based on the $635 \mathrm{~nm}$ peak wavelength LED635L (Thorlabs part LED635L) and the filter transmission spectrum is based on the $632.8 \mathrm{~nm}$ center wavelength bandpass filter FL632.8-1 (Thorlabs FL632.8-1). As the refractive index of the sensing layers, $n_{s}$, increase, the shifting reflectance dip decreases the intensity reading at the photodetector.

intensity-based readout for a specific dynamic range of the refractive index change corresponding to the typical concentrations of dissolved methane in seawater. To reach this goal, we proposed and evaluated multiple designs concepts, and revealed their advantages and disadvantages.

To the best of our knowledge, this is the first study offering the designs of the Bragg defect cavity and a Tamm plasmon perfect absorber optimized for use in planar dissolved methane sensor platforms with the intensity readout. Furthermore, this manuscript introduced a new conceptual design of a planar magnetic mirror with a multi-spectral response, which offers opportunities for ultra-high-sensitivity detection. All the new sensor designs presented here have been tailored to exhibit high sensitivity within the sensor dynamic range relevant for the dissolved methane sensing applications. Our results show that the planar Bragg reflector with a defect layer not only exhibits a large intensity response, but also is the most promising design for a low-cost and robust photonic chip for in-situ dissolved methane sensing. In the 
future, we plan to fabricate the Bragg reflector with a defect layer and characterize its response to dissolved methane. The Tamm and magnetic mirror structures should also be further evaluated for their promise in RI sensing. Importantly, all the sensor designs presented in this manuscript hold promise to outperform the state-of-theart surface plasmon resonance sensor when operated in the intensity readout mode without extra fabrication complexity or increased fabrication costs.

The application of the planar Bragg defect structure presented in this paper expands beyond dissolved methane sensing. Gas permeable nanostructures such as these could be explored for sensing other gases, either dissolved in water or present in the atmosphere. The sensing system proposed here avoids the need for costly equipment that is commonly used in other dissolved methane sensors, namely gas extraction systems and spectrometers [18]. Broadly, the implementation of spectrometer-free nanophotonic sensors in ocean sensing has the potential to shift the paradigm of ocean instruments from being large, bulky, and expensive towards small, portable, and affordable. 


\section{Chapter 4}

\section{Perspectives on Field-Deployable Microplastic Sensing}

An expanded version of this thesis chapter was accepted to MDPI's Sensors Journal at the time of this thesis submission, Blevins et al. 2021, and a shortened and reformatted version is reproduced here.

Chapters 2 and 3 have emphasized that accounting for cost, simplicity, and robustness in sensor design is key in pushing instrumentation from development in lab to actual application and measurement in the field. In this same vein, this chapter presents a thorough review of future perspectives in field-deployable microplastic sensing techniques. In this thesis chapter, MP measurement technologies are presented with a focus on both their eventual field-deployability and their respective data products (e.g. MP particle count, size, and/or polymer type).

\subsection{Introduction}

Microplastics (MPs) are small particles of polymer debris, commonly defined as being between $1 \mu \mathrm{m}$ and $1000 \mu \mathrm{m}$ [153], though no internationally agreed upon definition 
exists. MPs have emerged as an important subject of study for scientists with regards to their ecological impact and environmental fate and transport $[154,155]$. Research has been conducted to collect and analyze MPs from ponds [156], lakes [157, 158], rivers [158, 159], oceans [160-163], wastewater [164, 165], and drinking water [166]. Studies like these inform our understanding of the extent and impact of MPs in aqueous environments. However, open questions remain about the amount and distribution of MPs in our hydrosphere, as well as the environmental and ecological impacts of these particles [167,168]. Eriksen et al. (2014) estimated the total number of plastic particles in the ocean to be over 5 trillion, weighing over 250,000 tons; however, they acknowledge that the true concentration could be higher due to the limited number and distribution of available MP datasets [167], with little data available from the southern hemisphere [169-171]. Recent studies have found that MPs can absorb toxic compounds and transfer these to organisms that ingest them $[172,173]$. Other environmental and health consequences of MPs are still being studied, but results from these investigations are limited by a lack of MP sample data, due to the shortcomings of current sampling and analysis techniques $[168,174,175]$. It is clear that despite being accepted as a ubiquitous presence in aqueous environments, our ability to study MPs is hindered by a lack of technologies for rapidly characterizing environmental samples for MPs.

Currently, most open-water sample collections of MPs are conducted with plankton sampling nets, which are deployed from boats and ships $[172,176]$. Once collected, time-intensive laboratory work, including chemical pretreatment, is commonly required to prepare MP samples for analysis [172]. The American Society for Testing and Materials (ASTM) provides standards for these MP sample collection (D8332) [177] and preparation (D8333) [178] steps, however MP analysis technologies vary considerably and tend to be researcher-specific [179-181].

To provide a more thorough characterization of the MP pollution in aqueous environments (e.g. lakes, ponds, rivers, and oceans), MP analysis techniques must transition from manual laboratory approaches to robust and reproducible technologies that are well-suited for measurements in the field. Development of field-portable 
sensors will enable studies to achieve spatial coverage, sampling frequency, and time series data not possible with current techniques $[155,170]$. To enable development of field-deployable sensors, measurement techniques are needed that require minimal sample preparation so they can operate as in situ as possible.

To that end, this chapter identifies several measurement techniques that are potentially advantageous for application in a field-deployable MP sensor for aqueous environments and a related framework to evaluate them. A field-portable MP sensing system will require robust packaging, portable power electronics, and other components to be ready for field operation. Here the focus is restricted to technologies that would enable a critical measurement functionality and the full span of engineering efforts that will be needed to adapt the proposed technologies for field use, such as sample collection, are not addressed. Prata et al. (2019) present a comprehensive review of the current methods used for aqueous sample collection [182]. Each analysis technology is referred to as a "measurement technique", while "sensor" is used to describe an engineered instrument or system that incorporates one or more measurement techniques along with any collection or concentration technologies. Each measurement technique presented is characterized by the intrinsic physical property (chemical, mechanical, or electrical) it engages to distinguish MPs from their surroundings.

\subsection{Framework of Field-Deployable Microplastic Sens- ing}

This section introduces a two-part framework to evaluate the suitability of MP measurement techniques for use in field-portable sensors. The first part is a field-deployability tradespace, which establishes a set of criteria for comparing suitability for fielddeployability. The second part considers the measurement techniques themselves, how those techniques are coupled to the physical properties of MPs, and the specific data products that result. For instance, in this framework, what infrared microscopy 
may lack in field-deployability, it makes up for in the completeness of its MP characterization, delivering data regarding MP size, prevalence, morphology, and chemical type.

\subsubsection{Field-Deployability Tradespace}

Development of field-portable MP sensors will require thoughtful consideration of system design trade-offs. For instance, data quality may be associated with high cost and diminished durability. Users will have differing requirements for a field MP sensor, therefore this chapter presents a broad and pragmatic set of criteria for evaluating the field-deployability of a technology, but does not provide numerical scores for any specific technology. Users may weight the proposed criteria according to a specific application.

Field-deployable is defined here as having a favorable combination of the characteristics that would allow a sensor to operate in locations or on a platform where aqueous water samples are collected (e.g. on a boat, underwater vehicle, dock, or other location remote from the controlled environment of a laboratory). The characteristics proposed for consideration for a MP sensor in the field-deployability tradespace include: cost, durability, portability, low-power operation, fast-time response, and high-quality data (Figure 4-1). Additionally, this chapter considers the capability to analyze aqueous samples to be beneficial insofar as it avoids the need to chemically treat, dry, or spread extracted solids on a dry surface.

\subsubsection{Principles of Operation of MP Measurement Techniques and their Data Products}

Next we turn to the MP measurement techniques themselves, categorizing their principle of operation as chemical, mechanical, and electrical. For instance, techniques that rely on chemical properties are those that interact with the internal chemical structure of the sample. Many optical techniques fall in this category [183], for example, in

vibrational spectroscopic analysis, a unique reflectance, absorption, or transmission 
spectrum is obtained for a given MP type based on internal bond structure [184]. Analysis based on mechanical properties evaluate MP size, density, modulus of elasticity, and acoustic contrast factor [185]. Electrical properties of MPs include relative permittivity, and dielectrophoretic mobility [186, 187].

A.

MP Data Products

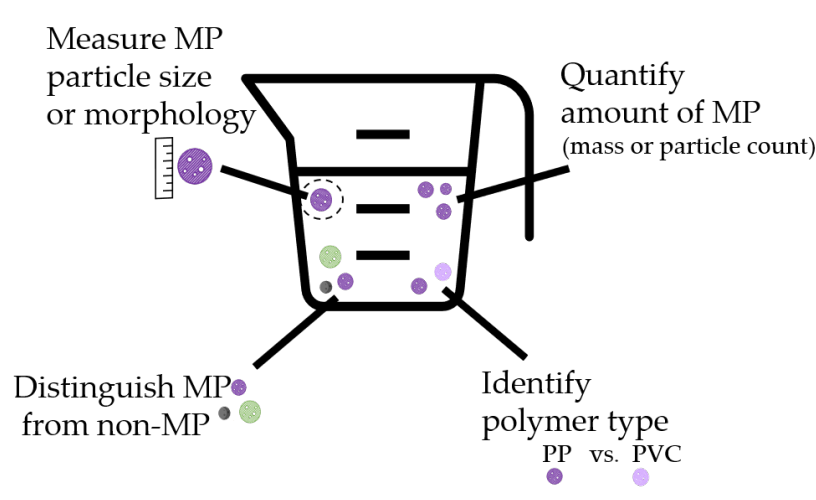

B. Field Deployable Metrics

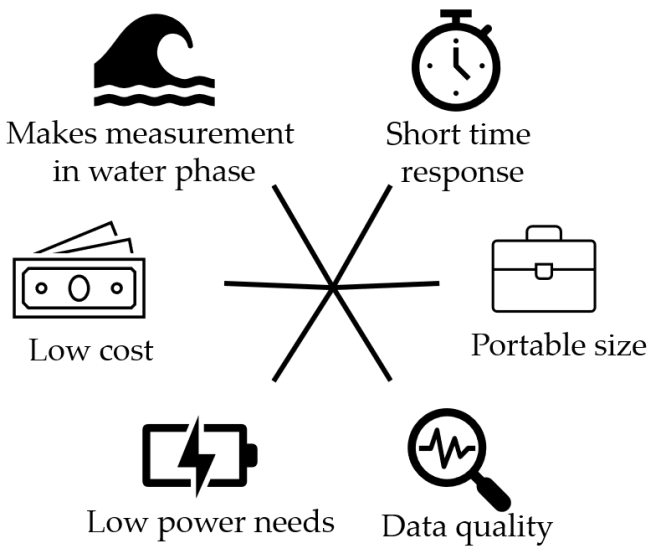

Figure 4-1: (A) Microplastic data products that can be collected from aqueous samples. MP number density, size distribution, mass, morphology, and adsorbed chemicals can all be further refined by measuring them by polymer type. (B) The key characteristics that define the field-deployable tradespace for a microplastic measurement technique for aqueous samples.

The most fundamental requirement of any MP sensor is a capability to positively identify MPs as polymers, as opposed to other non-MP particles in the environment, such as plankton, inorganic particles, and marine snow. This capability allows quantification of MP number density per sample volume. Ideally this number density could be broken down further by MP size distribution, polymer type, and morphology. Another requirement is the need to quantify the mass of MP particles in a sample, which can be derived from some combination of these measurements. Figure 4-1A illustrates the MP data products that can be collected from an aqueous sample. For completeness, identification of adsorbed chemicals is identified as a MP data product. Separate studies have addressed the measurement of adsorbed chemicals in MPs [188-190]. Combinations of these data products can be collected and analyzed to meet the varied objectives that exist for a MP study. 


\subsection{Review Results}

Table 4.1: Measurement techniques based on sample chemical properties with applicability to MP analysis. ${ }^{\dagger}$ Separation from Non-MPs is not a data product but instead an enabling technology for measuring MP data products.

*This observable has only preliminary data or is currently theoretical only.

\begin{tabular}{|c|c|c|c|}
\hline & Measurement & $\begin{array}{c}\text { Data } \\
\text { Products }\end{array}$ & Considerations for Field-Deployability \\
\hline \multirow{7}{*}{ 胥 } & Py-GC/MS & $\begin{array}{l}\text { Polymer } \\
\text { Type, } \\
\text { Relative } \\
\text { Mass }\end{array}$ & $\begin{array}{l}\text { Pyrolysis - Gas Chromatography / Mass Spectrometry (Py- } \\
\text { GC/MS). Demonstrated accuracy for MP polymer type iden- } \\
\text { tification (ID) [191-196]. Can characterize the identity of sam- } \\
\text { ple contaminants. Requires a dry sample, time-intensive data } \\
\text { collection and is often bulky. }\end{array}$ \\
\hline & $\begin{array}{c}\text { FTIR } \\
\text { Spectroscopy / } \\
\text { Imaging }\end{array}$ & $\begin{array}{c}\text { Polymer } \\
\text { Type, } \\
\text { Count, Size }\end{array}$ & $\begin{array}{l}\text { Fourier Transform Infrared Spectroscopy (FTIR). Demon- } \\
\text { strated accuracy for MP polymer type ID [197,198], often used } \\
\text { with attenuated total reflectance (ATR) [199]. Can character- } \\
\text { ize the identity of sample contaminants. Time-intensive due } \\
\text { to chemical pretreatment and scanning of dried sample sur- } \\
\text { face. Requires expensive, precisely aligned optomechanics, of- } \\
\text { ten bulky. Traditionally, sample must be dry. }\end{array}$ \\
\hline & $\begin{array}{c}\text { Raman } \\
\text { Spectroscopy / } \\
\text { Imaging }\end{array}$ & $\begin{array}{l}\text { Polymer } \\
\text { Type, Size, } \\
\text { Count }\end{array}$ & $\begin{array}{l}\text { Demonstrated accuracy for MP polymer type ID }[197,198,200- \\
202] \text {. Can characterize the identity of sample contaminants. } \\
\text { Time-intensive due to chemical pretreatment and scanning of } \\
\text { dried sample surface. Requires expensive, precisely aligned } \\
\text { optomechanics, often bulky. Traditionally, sample must be } \\
\text { dry. }\end{array}$ \\
\hline & $\begin{array}{l}\text { Hyperspectral } \\
\text { Imaging }\end{array}$ & $\begin{array}{c}\text { Polymer } \\
\text { Type, } \\
\text { Count, Size }\end{array}$ & $\begin{array}{l}\text { Demonstrated accuracy for MP polymer type ID in near- } \\
\text { infrared [203-205] or short-wave infrared [206] regimes. Can } \\
\text { characterize the identity of sample contaminants. Time- } \\
\text { intensive due to chemical pretreatment. Requires expensive, } \\
\text { precisely aligned optomechanics, often bulky. Traditionally, } \\
\text { sample must be dry. }\end{array}$ \\
\hline & Py-GC/DMS & $\begin{array}{l}\text { Polymer } \\
\text { Type, } \\
\text { Relative } \\
\text { Mass }\end{array}$ & $\begin{array}{l}\text { Pyrolysis - Gas Chromatography / Differential Mobility Spec- } \\
\text { trometry (Py-GC/DMS). Robust and portable package, cur- } \\
\text { rently used in non-MP field applications [207]. Can chemically } \\
\text { characterize the identity of sample contaminants. Lower cost } \\
\text { and smaller than Py-GC/MS. Requires a dry sample and time- } \\
\text { intensive data collection. Heritage as highly sensitive breath } \\
\text { diagnostic and air quality device. [207]. }\end{array}$ \\
\hline & $\begin{array}{l}\text { Multispectral } \\
\text { Imaging }\end{array}$ & $\begin{array}{c}\text { Polymer } \\
\text { Type, } \\
\text { Count, Size }\end{array}$ & $\begin{array}{l}\text { Rapid sample imaging. Time-intensive due to chemical pre- } \\
\text { treatment. Uses portable and relatively low-cost equipment } \\
\text { compared to spectrometers. Traditionally, sample must be dry. } \\
\text { Heritage in mineral and polymer type identification [208-210] }\end{array}$ \\
\hline & Fluorescent Dye & Count, Size & $\begin{array}{l}\text { Initial demonstrations with Nile Red }[211-214] \text { and pyrene } \\
{[215] \text { in laboratory MP studies. Uses low-cost equipment (dye, }} \\
\text { camera, and filter). May not require chemical pretreatment. } \\
\text { Potential for false positives }[216] \text {. Traditionally, sample must } \\
\text { be dry. }\end{array}$ \\
\hline
\end{tabular}


Table 4.1: Cont. Measurement techniques based on sample mechanical and electrical properties with applicability to MP analysis. ${ }^{\dagger}$ Separation from Non-MPs is not a data product but instead an enabling technology for measuring MP data products.

*This observable has only preliminary data or is currently theoretical only.

\begin{tabular}{|c|c|c|c|}
\hline \multirow{4}{*}{ 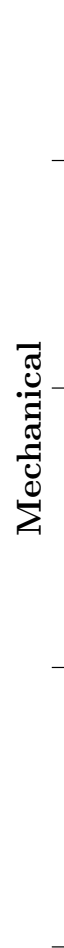 } & $\begin{array}{c}\text { Laser Optical } \\
\text { Trapping }\end{array}$ & $\begin{array}{l}\text { Separation } \\
\quad \text { from } \\
\text { Non-MPS }\end{array}$ & $\begin{array}{l}\text { Preliminary demonstrations of usefulness for MP identification } \\
\text { when coupled with Raman Spectroscopy }[217,218] \text {. Performed } \\
\text { in a microfluidic device, reducing sample preparation time. }\end{array}$ \\
\hline & $\begin{array}{l}\text { Photonic } \\
\text { Optical } \\
\text { Trapping }\end{array}$ & $\begin{array}{l}\text { Size }^{*} \\
\text { Separation } \\
\text { from } \\
\text { Non-MPs }\end{array}$ & $\begin{array}{l}\text { Performed in a microfluidic device, reducing sample prepara- } \\
\text { tion time. Heritage in particle sorting and manipulation for } \\
\text { bio-sensing and imaging [219-222]. }\end{array}$ \\
\hline & $\begin{array}{l}\text { Field Flow } \\
\text { Fractionation } \\
\qquad(\mathrm{FFF})\end{array}$ & $\begin{array}{l}\text { Size, } \\
\text { Separation } \\
\text { from } \\
\text { Non-MPs }\end{array}$ & $\begin{array}{l}\text { Centrifugal }[218] \text {, Asymmetrical flow }[218,223] \text {, or Thermal } \\
\text { [224]. A recent study used FFF with Raman Spectroscopy to } \\
\text { identify MP type [218]. Performed in a microfluidic device, re- } \\
\text { ducing sample preparation time. Equipment is relatively low- } \\
\text { cost and portable. }\end{array}$ \\
\hline & Acoustophoresis & $\begin{array}{l}\text { Polymer } \\
\text { Type*, Size, } \\
\text { Separation } \\
\text { from } \\
\text { Non-MPs }\end{array}$ & $\begin{array}{l}\text { Performed in a microfluidic device, reducing sample prepa- } \\
\text { ration time. Equipment is relatively low-cost and portable. } \\
\text { Heritage in cell and particle manipulation in microfluidics } \\
\text { field }[185,225] \text {. Recent studies have demonstrated MP sort- } \\
\text { ing }[226,227] \text {. }\end{array}$ \\
\hline \multirow{3}{*}{ : } & Ultrasound & $\begin{array}{c}\text { Polymer } \\
\text { Type*, Size* }\end{array}$ & $\begin{array}{l}\text { Performed in a microfluidic device or liquid volume, reducing } \\
\text { sample preparation time. Equipment is relatively low-cost and } \\
\text { portable. Heritage in flow cytometry }[228]\end{array}$ \\
\hline & $\begin{array}{l}\text { Impedance } \\
\text { Spectroscopy }\end{array}$ & $\begin{array}{l}\text { Polymer } \\
\text { Type*, } \\
\text { Count, Size }\end{array}$ & $\begin{array}{l}\text { Preliminary demonstrations of accuracy in MP identifica- } \\
\text { tion [187]. Performed in microfluidic device, reducing sam- } \\
\text { ple preparation time. Equipment is relatively low-cost and } \\
\text { portable [229]. }\end{array}$ \\
\hline & Dielectrophoresis & $\begin{array}{l}\text { Polymer } \\
\text { Type*, } \\
\text { Count, Size }\end{array}$ & $\begin{array}{l}\text { Performed in microfluidic devices, reducing sample preparation } \\
\text { time. Equipment is relatively low-cost and portable. Heritage } \\
\text { in cell and particle manipulation, some recent studies on use } \\
\text { with MPs }[186,230,231] \text {. }\end{array}$ \\
\hline
\end{tabular}

Table 4.1 compares a selection of prominent existing MP sensing technologies with several new or early-stage technologies. The tables consider each technology within the framework discussed, and describes notable features that are relevant to the framework. The measurement techniques are categorized by the basic 
properties they discern and are listed with the MP data products that they can assess/quantity/measure/observe (particle count and size, polymer type or relative mass of mixed polymer types).

Considering both the deployability trades (Figure 4-1) along with performance trades (Table 4.1), suggests that while FTIR may not be readily field-deployable, it is one of the few techniques that generates quality data of three desired data products (polymer type, size, count). Perhaps this is why FTIR and its vibrational spectroscopic analogue, Raman Microscopy, are the most widely used on samples collected for the laboratory and yet few studies show these methods performing in the field.

It is likely and perhaps inevitable that more than one technique can be combined to create a system which captures multiple MP characteristics for a specific application. For example, in recent studies, Raman Spectroscopy, which is normally performed on dry samples, was combined with optical trapping and microscopy to perform chemical identification and sizing of MPs in seawater [217,218]. In the study of micro-particle and organism, microscopy is often coupled with particle sorting and focusing technologies, for example, the FlowCAM couples flow cytometry with microscopy for precise imaging of micron scale specimens [232]. The combinations are numerous, and many possible way MP measurement techniques can be combined to target multiple MP characteristics, but here each is presented individually to motivate any combination of interest.

\subsection{Discussion}

This chapter seeks to inform the MP community of the potential of these techniques in creating rapid and automated MP sensors within a framework that addresses the full scope of practical/technological trade-offs to be considered. In addition to the MP measurement techniques presented here, development of efficient, portable MP sample collection and concentration technologies is equally crucial for creating full aqueous MP sensor systems. 
Rapid and portable MPs sensors would allow widespread monitoring at potential sources such as plastic production plants or waste water plants [233]. Technologies like these are essential for equipping scientists with the tools they need to better understand the fate, transport, abundance, and environmental impact of MPs in aqueous environments. Further, technologies like these are important for addressing the concerns of MP pollution and contamination in fisheries and aquaculture [234]. Effective, rapid, and affordable sensors are also integral in shaping and backing policies to limit MP contamination from factory run-off or keeping drinking water safe (e.g. California's Safe Drinking Water Act) [235]. 


\section{Chapter 5}

\section{Conclusion}

This thesis offers examples of and guidance on making dissolved gas monitoring more accessible and low-cost, pushing ocean and aqueous sensing into the domain of applied photonics, and driving microplastic detection techniques toward field deployment. This thesis will conclude with final thoughts on the future directions associated with the works in each chapter.

\subsection{The LC-GEMS for $\mathrm{pCO}_{2}$ Sensing}

Future iterations of the LC-GEMS presented in Chapter 2 can be incorporated with different gas sensors to enable low cost and accessible dissolved gas studies across a whole suite of targets. While the current LC-GEMS is designed for handheld used, structural changes to the LC-GEMS can adapt it for use on different platforms, such as incorporation on surface vehicles, ship flow-through systems, or on underwater vehicles. The design of the LC-GEMS is shared in this thesis so that community and research groups may replicate and iterate on it to conduct environmental studies that would otherwise require high-cost and bulky equipment. 


\subsection{Planar Nanophotonic Chips for dissolved $\mathrm{CH}_{4}$ Sensing}

The novel planar nanophotonic dissolved $\mathrm{CH}_{4}$ sensor designs presented in Chapter 3 are contributions to both the field of environmental instrumentation and the field of photonic sensing. Like the LC-GEMS, the proposal of the low-cost implementation of the Bragg defect structure for dissolved $\mathrm{CH}_{4}$ monitoring pushes the field of ocean sensing from large, expensive equipment to miniaturized, cheap instruments which can be multiplexed and deployed in a broad range of environments. Photonics, the study of light-matter interaction, is a field that emerged from the end of the 20th century and is now fueling progress in many technology sectors [105]. While some thin-film photonic techniques have been evaluated for ocean sensing applications, this thesis presents three new designs that motivate the further merging of photonics and ocean sensing. Broadly, the implementation of spectrometer-free nanophotonic sensors like those presented here for ocean sensing have the potential to shift the paradigm of ocean instruments from being large, bulky, and expensive towards small, portable, and affordable.

\subsection{Field Portable Microplastic Sensing}

The transition of the microplastic detection technologies presented in Chapter 4 from lab-based to field-based is key for obtaining the rich temporal and spatial data needed to better understand the fate, transport, and impact of MPs in aqueous environments. This thesis presents a review of potential future paths for field-deployable MP technologies, taking a novel perspective on the necessary next steps for advancing microplastic analysis. 


\subsection{Final words}

This thesis presents solutions for dissolved $\mathrm{CO}_{2}$ and $\mathrm{CH}_{4}$ sensing that address the need for more spatial and temporal data while factoring in the importance of real, practical applications of such sensors in the field. In this same vein, this thesis concludes with a review of the future perspectives in technology for field-depolyable microplastic sensing techniques. The efforts here highlight the essential collaboration and relationship needed between engineers and scientists to push new developments in instrumentation. Accounting for cost, simplicity, and robustness is key in pushing sensors from development and use in lab to actual application and measurement in the field. 


\section{Bibliography}

[1] Noaa Marine Debris Program. Strategic Plan NOAA Marine Debris Program 2 NOAA Marine Debris Program FY 2021-2025 Strategic Plan. Technical report, 2020.

[2] E.B. Jewett, E.B. Osborne, K.M. Arzayus, K. Osgood, J. DeAngelo, and J.M. Mintz. NOAA Ocean, Coastal, and Great Lakes Acidifcation Research Plan: 2020-2029. 2020.

[3] Ocean Acidification: A National Strategy to Meet the Challenges of a Changing Ocean. National Academies Press, 102010.

[4] Shivika Sharma and Subhankar Chatterjee. Microplastic pollution, a threat to marine ecosystem and human health: a short review. Environmental Science and Pollution Research, 24(27):21530-21547, 92017.

[5] Global Monitoring Laboratory US Department of Commerce, NOAA. Global Monitoring Laboratory - Carbon Cycle Greenhouse Gases.

[6] Wmo Unep, Neil Adger, Pramod Aggarwal, Shardul Agrawala, Joseph Alcamo, Abdelkader Allali, Oleg Anisimov, Nigel Arnell, Michel Boko, Osvaldo Canziani, Timothy Carter, Gino Casassa, Ulisses Confalonieri, Rex Victor Cruz, Edmundo De, Alba Alcaraz, William Easterling, Christopher Field, Andreas Fischlin, B Blair Fitzharris, Carlos Gay García, Clair Hanson, Hideo Harasawa, Kevin Hennessy, Saleemul Huq, Roger Jones, Kajfež Bogataj, David Karoly, Richard Klein, Zbigniew Kundzewicz, Murari Lal, Rodel Lasco, Geoff Love, Xianfu Lu, Graciela Magrín, Luis José Mata, Roger Mclean, Bettina Menne, Guy Midgley, 
Nobuo Mimura, Monirul Qader Mirza, José Moreno, Linda Mortsch, Isabelle Niang-Diop, Robert Nicholls, Béla Nováky, Leonard Nurse, Anthony Nyong, Michael Oppenheimer, Jean Palutikof, Martin Parry, Anand Patwardhan, Patricia Romero Lankao, Cynthia Rosenzweig, Stephen Schneider, Serguei Semenov, Joel Smith, John Stone, Jean-Pascal Van Ypersele, David Vaughan, Coleen Vogel, Thomas Wilbanks, Poh Wong, Shaohong Wu, and Gary Yohe. Intergovernmental Panel on Climate Change 2007: Impacts, Adaptation and Vulnerability Working Group II Contribution to the Intergovernmental Panel on Climate Change Fourth Assessment Report Summary for Policymakers Summary for Policymakers IPCC WGII Fourth Assessment Report. Technical report, 2007.

[7] S Solomon, D. Qin, M. Manning, Z. Chen, M. Marquis, K.B. Averyt, M.Tignor, and H.L. Miller. IPCC, 2007: Summary for Policymakers. In: Climate Change 2007: The Physical Science Basis. Contribution of Working \}Group I to the Fourth Assessment Report of the Intergovernmental Panel on Climate Change. Technical report, Cambridge University Press, Cambridge, United Kingdom and New York, NY, USA, 2007.

[8] C. Turley. Impacts of changing ocean chemistry in a high-CO 2 world . Mineralogical Magazine, 72(1):359-362, 22008.

[9] Matthew L. Kirwan and Simon M. Mudd. Response of salt-marsh carbon accumulation to climate change, 92012.

[10] John I. Hedges. Why Dissolved Organics Matter. In Biogeochemistry of Marine Dissolved Organic Matter, pages 1-33. Elsevier, 12002.

[11] Christian Lønborg, Cátia Carreira, Tim Jickells, and Xosé Antón ÁlvarezSalgado. Impacts of Global Change on Ocean Dissolved Organic Carbon (DOC) Cycling, 62020.

[12] Jordi Dachs and Laurence Méjanelle. Organic pollutants in coastal waters, sediments, and biota: A relevant driver for ecosystems during the anthropocene? Estuaries and Coasts, 33(1):1-14, 12010. 
[13] Richard G. Williams and Michael J. Follows. Ocean Dynamics and The Carbon Cycle: Principles and Mechanisms. Cambridge University Press, 2011.

[14] Allen Doyle, Michael N. Weintraub, and Joshua P. Schimel. Persulfate Digestion and Simultaneous Colorimetric Analysis of Carbon and Nitrogen in Soil Extracts. Soil Science Society of America Journal, 68(2):669-676, 32004.

[15] Randall Kolka, Peter Weisbarnpel, and Mats Froberg. Measurement and Importance of Dissolved Organic Carbon. Technical report.

[16] Carolyn D. Ruppel and John D. Kessler. The interaction of Climate Change and Methane Hydrates. Deep-Sea Research Part II: Topical Studies in Oceanography, 57(1):1-20, 2016.

[17] Lisa A. Levin, Amy R. Baco, David A. Bowden, Ana Colaco, Erik E. Cordes, Marina R. Cunha, Amanda W.J. Demopoulos, Judith Gobin, Benjamin M. Grupe, Jennifer Le, Anna Metaxas, Amanda N. Netburn, Greg W. Rouse, Andrew R. Thurber, Verena Tunnicliffe, Cindy Lee Van Dover, Ann Vanreusel, and Les Watling. Hydrothermal vents and methane seeps: Rethinking the sphere of influence. Frontiers in Marine Science, 3(MAY):72, 52016.

[18] C Boulart, D P Connelly, and M C Mowlem. Sensors and technologies for in situ dissolved methane measurements and their evaluation using Technology Readiness Levels. TrAC Trends in Analytical Chemistry, 29(2):186-195, 2010.

[19] A.G. Dickson, C.L. Sabine, and J.R Christian. Guide to Best Practices for Ocean C0 2 Measurements. PICES Special Publication . Technical report, 2007.

[20] J. Zosel, W. Oelner, M. Decker, G. Gerlach, and U. Guth. The measurement of dissolved and gaseous carbon dioxide concentration, 52011.

[21] Steven Emerson and John Hedges. Chemical oceanography and the marine carbon cycle, volume 9780521833134. Cambridge University Press, 12008. 
[22] Emilio Mayorga, Anthony K. Aufdenkampe, Caroline A. Masiello, Alex V. Krusche, John I. Hedges, Paul D. Quay, Jeffrey E. Richey, and Thomas A. Brown. Young organic matter as a source of carbon dioxide outgassing from Amazonian rivers. Nature, 436(7050):538-541, 72005.

[23] A P McNichol, P D Quay, A R Gagnon, and J R Burton. Collection and Measurement of Carbon Isotopes in Seawater DIC. Technical report, 2010.

[24] Zeland Muccio and Glen P. Jackson. Isotope ratio mass spectrometry, 2009.

[25] Alex Bayliss, Gerry Mccormac, and Hans Van Der Plicht. An illustrated guide to measuring radiocarbon from archaeological samples. Technical report, 2004.

[26] Terhi Rasilo, Yves T. Prairie, and Paul A. del Giorgio. Large-scale patterns in summer diffusive $\mathrm{CH} 4$ fluxes across boreal lakes, and contribution to diffusive C emissions. Global Change Biology, 21(3):1124-1139, 32015.

[27] Audrey Campeau and Paul A. Del Giorgio. Patterns in CH4 and CO2 concentrations across boreal rivers: Major drivers and implications for fluvial greenhouse emissions under climate change scenarios. Global Change Biology, 20(4):10751088, 42014.

[28] J. F. Magenheimer, T. R. Moore, G. L. Chmura, and R. J. Daoust. Methane and carbon dioxide flux from a macrotidal salt marsh, Bay of Fundy, New Brunswick. Estuaries, 19(1):139-145, 1996.

[29] Gwenaël Abril and Alberto Vieira Borges. Carbon Dioxide and Methane Emissions from Estuaries. pages 187-207. Springer, Berlin, Heidelberg, 2005.

[30] Changchun Song, Jinbo Zhang, Yiyong Wang, Yaosi Wang, and Zhichun Zhao. Emission of $\mathrm{CO} 2, \mathrm{CH} 4$ and $\mathrm{N} 2 \mathrm{O}$ from freshwater marsh in northeast of China. Journal of Environmental Management, 88(3):428-436, 82008.

[31] A. M. Nahlik and M. S. Fennessy. Carbon storage in US wetlands. Nature Communications, 7(1):1-9, 122016. 
[32] A. J. Williams. CTD (conductivity, temperature, depth) profiler. In Encyclopedia of Ocean Sciences, pages 1-10. Elsevier, 12019.

[33] Ocean Instruments : Woods Hole Oceanographic Institution. https://www.whoi.edu/science/instruments/.

[34] Cédric Magen, Laura L. Lapham, John W. Pohlman, Kathleen Marshall, Samantha Bosman, Michael Casso, and Jeffrey P. Chanton. A simple headspace equilibration method for measuring dissolved methane. Limnology and Oceanography: Methods, 12(SEP):637-650, 2014.

[35] Taro Takahashi, Jon Olafsson, John G. Goddard, David W. Chipman, and S. C. Sutherland. Seasonal variation of $\mathrm{CO} 2$ and nutrients in the high-latitude surface oceans: A comparative study. Global Biogeochemical Cycles, 7(4):843878, 1993.

[36] Ingeborg Bussmann, Irina Fedorova, Bennet Juhls, Pier Paul Overduin, and Matthias Winkel. Methane dynamics in three different Siberian water bodies under winter and summer conditions. Biogeosciences, 18(6):2047-2061, 32021.

[37] Christopher S. Martens and J. Val Klump. Biogeochemical cycling in an organic-rich coastal marine basin-I. Methane sediment-water exchange processes. Geochimica et Cosmochimica Acta, 44(3):471-490, 31980.

[38] J. A. Leach, A. Larsson, M. B. Wallin, M. B. Nilsson, and H. Laudon. Twelve year interannual and seasonal variability of stream carbon export from a boreal peatland catchment. Journal of Geophysical Research: Biogeosciences, 121(7):1851-1866, 72016.

[39] Vallero. Daniel. Fundamentals of Air Pollution, volume 5. Elsevier, 2014.

[40] O. David Sparkman, Zelda Penton, and Fulton G. Kitson. Gas Chromatography and Mass Spectrometry: A Practical Guide, volume 2. Academic Press, 2011. 
[41] Raymond H. Hesslein. An in situ sampler for close interval pore water studies1. Limnology and Oceanography, 21(6):912-914, 111976.

[42] C Barber and D Briegel. A Method for the in-situ Determination of Dissolved Methane in Groundwater in Shallow Aquifers. Technical report, 1987.

[43] Jackie R. Webb, Damien T. Maher, and Isaac R. Santos. Automated, in situ measurements of dissolved $\mathrm{CO} 2, \mathrm{CH} 2$, and $\delta 13 \mathrm{C}$ values using cavity enhanced laser absorption spectrometry: Comparing response times of air-water equilibrators. Limnology and Oceanography: Methods, 14(5):323-337, 52016.

[44] Jimmy Liem, Tom Owano, Manish Gupta, Douglas Baer Los, and Gatos Research. Novel System for Continuous Measurements of Dissolved Gases in Liquids. Technical report.

[45] R. F. Weiss. Determinations of Carbon Dioxide and Methane by Dual Catalyst Flame Ionization Chromatography and Nitrous Oxide by Electron Capture Chromatography. Journal of Chromatographic Science, 19(12):611-616, 12 1981.

[46] Arne Körtzinger, Helmuth Thomas, Bernd Schneider, Nicole Gronau, Ludger Mintrop, and Jan C. Duinker. At-sea intercomparison of two newly designed underway pCO2 systems - Encouraging results. Marine Chemistry, 52(2):133145, 41996.

[47] R. Krishna and J. M. Van Baten. Mass transfer in bubble columns. In Catalysis Today, volume 79-80, pages 67-75. Elsevier, 42003.

[48] Michel Frankignoulle, Alberto Borges, and Renzo Biondo. A new design of equilibrator to monitor carbon dioxide in highly dynamic and turbid environments. Water Research, 35(5):1344-1347, 42001.

[49] Elizabeth A Bagshaw, Alexander Beaton, Jemma L Wadham, Matt Mowlem, Jon R Hawkings, and Martyn Tranter. Chemical sensors for in situ data col- 
lection in the cryosphere. TrAC Trends in Analytical Chemistry, 82:348-357, 2016.

[50] David P Nicholson, Anna P M Michel, Scott D Wankel, Kevin Manganini, Rebecca A Sugrue, Zoe O Sandwith, and Samuel A Monk. Rapid Mapping of Dissolved Methane and Carbon Dioxide in Coastal Ecosystems Using the ChemYak Autonomous Surface Vehicle. Environmental Science 85 Technology, 52(22):13314-13324, 2018.

[51] Anna P.M. Michel, Scott D. Wankel, Jason Kapit, Zoe Sandwith, and Peter R. Girguis. In situ carbon isotopic exploration of an active submarine volcano. Deep-Sea Research Part II: Topical Studies in Oceanography, 150:57-66, 42018.

[52] Cara C Manning, Victoria L Preston, Samantha F Jones, Anna P M Michel, David P Nicholson, Patrick J Duke, Mohamed M M Ahmed, Kevin Manganini, Brent G T Else, and Philippe D Tortell. River Inflow Dominates Methane Emissions in an Arctic Coastal System. Geophysical Research Letters, 47(10):e2020GL087669, 2020.

[53] ABB - Los Gatos Research. Los Gatos Research Greenhouse Gas Analyzer (CH4,CO2,H2O)-Ultraportable. http://www.lgrinc.com/analyzers/ultraportable-greenhouse-gas-analyzer/.

[54] Scott D Wankel, Yi-wen Huang, Manish Gupta, Robert Provencal, J Brian Leen, Andrew Fahrland, Charles Vidoudez, and Peter R Girguis. Characterizing the Distribution of Methane Sources and Cycling in the Deep Sea via in Situ Stable Isotope Analysis. Environmental Science 83 Technology, 47(3):1478-1486, 22013.

[55] Pro-Oceanus Systems Inc. Mini CH 4 Digital Model User's Manual. Technical report, www.pro-oceanus.com, 2016.

[56] Kongsberg Maritime. CONTROS HydroC CH 4 Operating principle. Technical report, 102018. 
[57] Jonathan J. Cole, Nina F. Caraco, George W. Kling, and Timothy K. Kratz. Carbon dioxide supersaturation in the surface waters of lakes. Science, 265(5178):1568-1570, 91994.

[58] Sylvie Pighini, Maurizio Ventura, Franco Miglietta, and Georg Wohlfahrt. Dissolved greenhouse gas concentrations in 40 lakes in the Alpine area. Aquatic Sciences, 80(3):32, 72018.

[59] Jake J. Beaulieu, Sarah Waldo, David A. Balz, Will Barnett, Alexander Hall, Michelle C. Platz, and Karen M. White. Methane and Carbon Dioxide Emissions From Reservoirs: Controls and Upscaling. Journal of Geophysical Research: Biogeosciences, 125(12), 122020.

[60] Gail L. Chmura, Shimon C. Anisfeld, Donald R. Cahoon, and James C. Lynch. Global carbon sequestration in tidal, saline wetland soils. Global Biogeochemical Cycles, 17(4):n/a-n/a, 122003.

[61] I. Forbrich, A. E. Giblin, and C. S. Hopkinson. Constraining Marsh Carbon Budgets Using Long-Term C Burial and Contemporary Atmospheric CO $<$ sub $>2</$ sub $>$ Fluxes. Journal of Geophysical Research: Biogeosciences, 123(3):867-878, 32018.

[62] Luc Pelletier, Ian B. Strachan, Nigel T. Roulet, Michelle Garneau, and Karoline Wischnewski. Effect of open water pools on ecosystem scale surface-atmosphere carbon dioxide exchange in a boreal peatland. Biogeochemistry, 124(1-3):291304,52015 .

[63] Hafsah Nahrawi, Monique Y Leclerc, Gengsheng Zhang, and Roshani Pahari. Influence of Tidal Inundation on CO2 Exchange between Salt Marshes and the Atmosphere.

[64] Michel Frankignoulle, Gwenaël Abril, Alberto Borges, Isabelle Bourge, Christine Canon, Bruno Delille, Emile Libert, and Jean Marie Théate. Carbon dioxide emission from European estuaries, 101998. 
[65] John T. Crawford, Luke C. Loken, Nora J. Casson, Colin Smith, Amanda G. Stone, and Luke A. Winslow. High-speed limnology: Using advanced sensors to investigate spatial variability in biogeochemistry and hydrology. Environmental Science and Technology, 49(1):442-450, 12015.

[66] Technical Note 1.1: Dissolved CO2 and Units of Measurement. ProOceanus.com.

[67] Andrew H. Manning, D. Solomon, and Amy L Sheldon. Applications of a Total Dissolved Gas Pressure Probe in Ground Water Studies. Ground Water, 41(4):440-448, 72003.

[68] Shangbin Xiao, Liu Liu, Wei Wang, Andreas Lorke, and Jason Woodhouse. A Fast-Response Automated Gas Equilibrator (FaRAGE) for continuous in situ measurement of CH4 and CO2 dissolved in water. Hydrology and Earth System Sciences, 24(7):3871-3880, 72020.

[69] Shangbin Xiao, Liu Liu, and Hans-Peter Grossart. Supplement of A FastResponse Automated Gas Equilibrator (FaRAGE) for continuous in situ measurement of $\mathrm{CH} 4$ and $\mathrm{CO} 2$ dissolved in water The copyright of individual parts of the supplement might differ from the CC BY 4.0 License. Supplement of Hydrol. Earth Syst. Sci, 24:3871-3880, 2020.

[70] Kazuo Nakamoto. Infrared and Raman Spectra of Inorganic and Coordination Compounds. John Wiley \& Sons, Inc., Hoboken, NJ, USA, 122008.

[71] Daniel C Harris. Symmetry and Spectroscopy: An Introduction to Vibrational and Electronic Spectrscopy. Technical report, 1978.

[72] Peter R. Griffiths and James A. De Haseth. Fourier Transform Infrared Spectrometry. John Wiley \& Sons, Inc, 2 edition, 2007.

[73] You Wen Sun, Yi Zeng, Wen Qing Liu, Pin Hua Xie, Ka Lok Chan, Xian Xin Li, Shi Mei Wang, and Shu Hua Huang. Cross-interference correction and 
simultaneous multi-gas analysis based on infrared absorption. Chinese Physics B, 21(9):090701, 92012.

[74] Trieu Vuong Dinh, In Young Choi, Youn Suk Son, and Jo Chun Kim. A review on non-dispersive infrared gas sensors: Improvement of sensor detection limit and interference correction, 82016 .

[75] Tomomi Yasuda, Seiichiro Yonemura, and Akira Tani. Comparison of the characteristics of small commercial NDIR CO2 sensor models and development of a portable CO2 measurement device. Sensors, 12(3):3641-3655, 32012.

[76] Stephen D. Piccot, Sushma S. Masemore, Eric S. Srinivasan, David A. Kirchgessner, and William F. Herget. Validation of a method for estimating pollution emission rates from area sources using open-path ftir spectroscopy anddispersion modeling techniques. Air and Waste, 44(3):271-279, 1994.

[77] Cory R. Martin, Ning Zeng, Anna Karion, Russell R. Dickerson, Xinrong Ren, Bari N. Turpie, and Kristy J. Weber. Evaluation and environmental correction of ambient CO2 measurements from a low-cost NDIR sensor. Atmospheric Measurement Techniques, 10(7):2383-2395, 72017.

[78] George F. Fine, Leon M. Cavanagh, Ayo Afonja, and Russell Binions. Metal Oxide Semi-Conductor Gas Sensors in Environmental Monitoring. Sensors, 10(6), 62010.

[79] Chengxiang Wang, Longwei Yin, Luyuan Zhang, Dong Xiang, and Rui Gao. Metal oxide gas sensors: Sensitivity and influencing factors, 32010.

[80] Luciano Mendes, Nico Ogink, Nadège Edouard, Hendrik van Dooren, Ilda Tinôco, and Julio Mosquera. NDIR Gas Sensor for Spatial Monitoring of Carbon Dioxide Concentrations in Naturally Ventilated Livestock Buildings. Sensors, 15(5):11239-11257, 52015. 
[81] Sudhir Pandey and Ki-Hyun Kim. The Relative Performance of NDIR-based Sensors in the Near Real-time Analysis of CO2 in Air. Sensors, 7(9):1683-1696, 92007.

[82] Peer Fietzek, Björn Fiedler, Tobias Steinhoff, and Arne Körtzinger. In situ quality assessment of a novel underwater pCO 2 sensor based on membrane equilibration and NDIR spectrometry. Journal of Atmospheric and Oceanic Technology, 31(1):181-196, 2014.

[83] Leah Bandstra, Burke Hales, and Taro Takahashi. High-frequency measurements of total CO2: Method development and first oceanographic observations. Marine Chemistry, 100(1-2):24-38, 62006.

[84] Mark S. Johnson, Michael F. Billett, Kerry J. Dinsmore, Marcus Wallin, Kirstie E. Dyson, and Rachhpal S. Jassal. Direct and continuous measurement of dissolved carbon dioxide in freshwater aquatic systems-method and applications. Ecohydrology, 3(1):68-78, 32010.

[85] Nguyen Thanh Duc, Samuel Silverstein, Martin Wik, Patrick Crill, David Bastviken, and Ruth K. Varner. Technical note: Greenhouse gas flux studies: An automated online system for gas emission measurements in aquatic environments. Hydrology and Earth System Sciences, 24(7):3417-3430, 72020.

[86] S. L. Brown, C. S. Goulsbra, M. G. Evans, T. Heath, and E. Shuttleworth. Low cost CO2 sensing: A simple microcontroller approach with calibration and field use. HardwareX, 8:e0136, 102020.

[87] Samuel Gagnon, Emmanuel L'Hérault, Mickaël Lemay, and Michel Allard. New low-cost automated system of closed chambers to measure greenhouse gas emissions from the tundra. Agricultural and Forest Meteorology, 228-229:29-41, 11 2016.

[88] Datasheet: K-30 Sensor. CO2meter.com, 52015. 
[89] John Colt. Solubility of Atmospheric Gases in Freshwater. In Computation of Dissolved Gas Concentration in Water as Functions of Temperature, Salinity and Pressure, pages 1-71. Elsevier, 12012.

[90] Mahmoud Al-Hindi and Fouad Azizi. Absorption and desorption of carbon dioxide in several water types. Canadian Journal of Chemical Engineering, 96(1):274-284, 72018.

[91] Damian Moran. Carbon dioxide degassing in fresh and saline water. I: Degassing performance of a cascade column. Aquacultural Engineering, 43(1):29-36, 7 2010.

[92] Shiyu Rachel Wang, Daniela Di Iorio, Wei Jun Cai, and Charles S. Hopkinson. Inorganic carbon and oxygen dynamics in a marsh-dominated estuary. Limnology and Oceanography, 63(1):47-71, 12018.

[93] Douglas C Montgomery, George C Runger, and John Wiley. Applied Statistics and Probability for Engineers Third Edition. Technical report, 2003.

[94] Nathaniel E Helwig. Multivariate Linear Regression. Technical report, 2017.

[95] Wanda Gülzow, Gregor Rehder, Bernd Schneider, Jens Schneider v. Deimling, and Bernd Sadkowiak. A new method for continuous measurement of methane and carbon dioxide in surface waters using off-axis integrated cavity output spectroscopy (ICOS): An example from the Baltic Sea. Limnology and Oceanography: Methods, 9(5):176-184, 52011.

[96] C-sense pCO2 Sensor | Turner Designs | United States. https://www.turnerdesigns.com/c-sense-in-situ-pco2-sensor.

[97] Mitsuru Hirota, Yukiko Senga, Yasushi Seike, Seiichi Nohara, and Hidenobu Kunii. Fluxes of carbon dioxide, methane and nitrous oxide in two contrastive fringing zones of coastal lagoon, Lake Nakaumi, Japan. Chemosphere, 68(3):597-603, 62007. 
[98] S. H. Knox, L. Windham-Myers, F. Anderson, C. Sturtevant, and B. Bergamaschi. Direct and Indirect Effects of Tides on Ecosystem-Scale CO2 Exchange in a Brackish Tidal Marsh in Northern California. Journal of Geophysical Research: Biogeosciences, 123(3):787-806, 32018.

[99] James T. Morris and Gary J. Whiting. Emission of gaseous carbon dioxide from salt-marsh sediments and its relation to other carbon losses. Estuaries, $9(1): 9-19,31986$.

[100] Chuan Tong, Jia F. Huang, Zhi Q. Hu, and Yu F. Jin. Diurnal Variations of Carbon Dioxide, Methane, and Nitrous Oxide Vertical Fluxes in a Subtropical Estuarine Marsh on Neap and Spring Tide Days. Estuaries and Coasts, 36(3):633-642, 52013.

[101] Joshua M. Blackstock, Matthew D. Covington, Matija Perne, and Joseph M. Myre. Monitoring Atmospheric, Soil, and Dissolved CO2 Using a Low-Cost, Arduino Monitoring Platform (CO2-LAMP): Theory, Fabrication, and Operation. Frontiers in Earth Science, 7:313, 112019.

[102] Tae Kyung Yoon, Hyojin Jin, Neung Hwan Oh, and Ji Hyung Park. Technical note: Assessing gas equilibration systems for continuous pCO2 measurements in inland waters. Biogeosciences, 13(13):3915-3930, 72016.

[103] Enric Sala, Juan Mayorga, Darcy Bradley, Reniel B. Cabral, Trisha B. Atwood, Arnaud Auber, William Cheung, Christopher Costello, Francesco Ferretti, Alan M. Friedlander, Steven D. Gaines, Cristina Garilao, Whitney Goodell, Benjamin S. Halpern, Audra Hinson, Kristin Kaschner, Kathleen KesnerReyes, Fabien Leprieur, Jennifer McGowan, Lance E. Morgan, David Mouillot, Juliano Palacios-Abrantes, Hugh P. Possingham, Kristin D. Rechberger, Boris Worm, and Jane Lubchenco. Protecting the global ocean for biodiversity, food and climate. Nature, 592(7854):397-402, 42021. 
[104] Morgan G. Blevins, Anna P. M. Michel, and Svetlana V. Boriskina. Planar nanophotonic structures for intensity based readout refractive index sensing applied to dissolved methane detection. OSA Continuum, 3(12):3556, 122020.

[105] John D. J Joannopoulos, Steven G. Johnson, Joshua N. Winn, and Robert D. Meade. Photonic Crystals: Molding the Flow of Light. Princeton University Press, 2 edition, 32008.

[106] Charles D Koven, Bruno Ringeval, Pierre Friedlingstein, Philippe Ciais, Patricia Cadule, Dmitry Khvorostyanov, Gerhard Krinner, and Charles Tarnocai. Permafrost carbon-climate feedbacks accelerate global warming. Proceedings of the National Academy of Sciences, 108(36):14769-14774, 2011.

[107] Joshua F Dean, Jack J Middelburg, Thomas Röckmann, Rien Aerts, Luke G Blauw, Matthias Egger, Mike S M Jetten, Anniek E E de Jong, Ove H Meisel, Olivia Rasigraf, Caroline P Slomp, Michiel H in't Zandt, and A J Dolman. Methane Feedbacks to the Global Climate System in a Warmer World. Reviews of Geophysics, 56(1):207-250, 2018.

[108] Katrin Kohnert, Andrei Serafimovich, Stefan Metzger, Jörg Hartmann, and Torsten Sachs. Strong geologic methane emissions from discontinuous terrestrial permafrost in the Mackenzie Delta, Canada. Scientific Reports, 7(1):5828, 2017.

[109] Laurent Garel, Jean-Pierre Dutasta, and André Collet. Complexation of Methane and Chlorofluorocarbons by Cryptophane-A in Organic Solution. Angewandte Chemie International Edition in English, 32(8):1169-1171, 1993.

[110] Stefania Torino, Laura Conte, Mario Iodice, Giuseppe Coppola, and Ralf D Prien. PDMS membranes as sensing element in optical sensors for gas detection in water. Sensing and Bio-Sensing Research, 16:74-78, 2017.

[111] Cédric Boulart, Matthew C Mowlem, Douglas P Connelly, Jean-Pierre Dutasta, and Christopher R German. A novel, low-cost, high performance dissolved methane sensor for aqueous environments. Optics Express, 16(17):12607, 2008. 
[112] Cédric Boulart, Ralf Prien, Valérie Chavagnac, and Jean-Pierre Dutasta. Sensing Dissolved Methane in Aquatic Environments: An Experiment in the Central Baltic Sea Using Surface Plasmon Resonance. Environmental Science \& Technology, page 130716153115002, 2013.

[113] H Apriyanto, O Bernal, F Lizion, F Surre, J H Sharp, V Chavagnac, and H C Seat. Optical fiber methane sensor using refractometry. In 2019 IEEE SENSORS, pages 1-4. IEEE, 2019.

[114] Firehun Tsige Dullo, Susan Lindecrantz, Jana Jágerská, Jørn H Hansen, Magnus Engqvist, Stian Andre Solbø, and Olav Gaute Hellesø. Sensitive on-chip methane detection with a cryptophane-A cladded Mach-Zehnder interferometer. Optics Express, 23(24):31564, 2015.

[115] Suozhu Wu, Yan Zhang, Zhongping Li, Shaomin Shuang, Chuan Dong, and Martin M.F. Choi. Mode-filtered light methane gas sensor based on cryptophane A. Analytica Chimica Acta, 633(2):238-243, 22009.

[116] Jianchun Yang, Lang Zhou, Jing Huang, Chuanyi Tao, Xueming Li, and Weimin Chen. Sensitivity enhancing of transition mode long-period fiber grating as methane sensor using high refractive index polycarbonate/cryptophane A overlay deposition. Sensors and Actuators B: Chemical, 207:477-480, 2015.

[117] Mauricio E Calvo and Hernán Míguez. Flexible, Adhesive, and Biocompatible Bragg Mirrors Based on Polydimethylsiloxane Infiltrated Nanoparticle Multilayers. Chemistry of Materials, 22(13):3909-3915, 2010.

[118] Leonid M Goldenberg, Victor Lisinetskii, and Sigurd Schrader. Fast and simple fabrication of organic Bragg mirrors - application to plastic microchip lasers. Laser Physics Letters, 10(5):055808, 2013.

[119] Arif E Cetin, Ahmet F Coskun, Betty C Galarreta, Min Huang, David Herman, Aydogan Ozcan, and Hatice Altug. Hand-Held Plasmonic Biosensor for HighThroughput Sensing for Point-of-Care Applications. 2014. 
[120] Yousef Nazirizadeh, Uwe Bog, Sylwia Sekula, Timo Mappes, Uli Lemmer, and Martina Gerken. Low-cost label-free biosensors using photonic crystals embedded between crossed polarizers. Optics Express, 18(18):19120, 82010.

[121] Sabrina Jahns, Marion Bräu, Björn-Ole Meyer, Torben Karrock, Sören B Gutekunst, Lars Blohm, Christine Selhuber-Unkel, Raymund Buhmann, Yousef Nazirizadeh, and Martina Gerken. Handheld imaging photonic crystal biosensor for multiplexed, label-free protein detection. Biomedical Optics Express, 6(10):3724-3736, 2015.

[122] David Michel, Feng Xiao, and Kamal Alameh. A compact, flexible fiber-optic Surface Plasmon Resonance sensor with changeable sensor chips. Sensors and Actuators B: Chemical, 246:258-261, 2017.

[123] T. M. Chinowsky, J. G. Quinn, D. U. Bartholomew, R. Kaiser, and J. L. Elkind. Performance of the Spreeta 2000 integrated surface plasmon resonance affinity sensor. Sensors and Actuators, B: Chemical, 91(1-3):266-274, 62003.

[124] Yi Xu, Ping Bai, Xiaodong Zhou, Yuriy Akimov, Ching Eng Png, Lay-Kee Ang, Wolfgang Knoll, and Lin Wu. Optical Refractive Index Sensors with Plasmonic and Photonic Structures: Promising and Inconvenient Truth. Advanced Optical Materials, 7(9):1801433, 52019.

[125] James F. Shackelford, Young-Hwan Han, Sukyoung Kim, and Se-Hun Kwon. CRC Materials Scienece and Engineering Handbook (4th ed.). CRC Press, fourth edi edition, 2015.

[126] Jiankun Peng, Weijia Wang, Yapeng Qu, Tengpeng Sun, Dajuan Lv, Jixiang Dai, and Minghong Yang. Thin films based one-dimensional photonic crystal for humidity detection. Sensors and Actuators A: Physical, 263:209-215, 2017.

[127] Antoine R M Dalod, Ola G Grendal, Anders B Blichfeld, Vedran Furtula, Javier Pérez, Lars Henriksen, Tor Grande, and Mari-Ann Einarsrud. Structure and 
Optical Properties of Titania-PDMS Hybrid Nanocomposites Prepared by In Situ Non-Aqueous Synthesis. Nanomaterials, 7(12):460, 2017.

[128] Mark Portnoi, Thomas J Macdonald, Christian Sol, Tom S Robbins, Tao Li, Johannes Schläfer, Stefan Guldin, Ivan P Parkin, and Ioannis Papakonstantinou. All-Silicone-based Distributed Bragg Reflectors for Efficient Flexible Luminescent Solar Concentrators. Nano Energy, 70:104507, 2020.

[129] Michael F Weber, Carl A Stover, Larry R Gilbert, Timothy J Nevitt, and Andrew J Ouderkirk. Giant Birefringent Optics in Multilayer Polymer Mirrors. Science, 287(5462):2451, 32000.

[130] James Giammarco, Bogdan Zdyrko, Laeticia Petit, J. David Musgraves, Juejun Hu, Anu Agarwal, Lionel Kimerling, Kathleen Richardson, and Igor Luzinov. Towards universal enrichment nanocoating for IR-ATR waveguides. Chemical Communications, 47(32):9104-9106, 82011.

[131] M. E. Sasin, R. P. Seisyan, M. A. Kalitteevski, S. Brand, R. A. Abram, J. M. Chamberlain, A. Yu Egorov, A. P. Vasil'Ev, V. S. Mikhrin, and A. V. Kavokin. Tamm plasmon polaritons: Slow and spatially compact light. Applied Physics Letters, 92(25):251112, 62008.

[132] Baptiste Auguié, María Cecilia Fuertes, Paula C. Angelomé, Nicolás López Abdala, Galo J.A.A. Soler Illia, and Alejandro Fainstein. Tamm Plasmon Resonance in Mesoporous Multilayers: Toward a Sensing Application. ACS Photonics, 1(9):775-780, 92014.

[133] Yoichiro Tsurimaki, Jonathan K. Tong, Victor N. Boriskin, Alexander Semenov, Mykola I. Ayzatsky, Yuri P. MacHekhin, Gang Chen, and Svetlana V. Boriskina. Topological Engineering of Interfacial Optical Tamm States for Highly Sensitive Near-Singular-Phase Optical Detection. ACS Photonics, 5(3):929-938, 32018. 
[134] Svetlana V Boriskina and Yoichiro Tsurimaki. Sensitive singular-phase optical detection without phase measurements with Tamm plasmons. Journal of Physics Condensed Matter, 30(22), 52018.

[135] Samir Kumar and Ritwick Das. On the tunability of quality-factor for optical Tamm plasmon modes. Journal of Optics (United Kingdom), 19(9):095001, 7 2017.

[136] Dan Sievenpiper, Lijun Zhang, Romulo F. Jimenez Broas, Nicholas G. Alexöpolous, and Eli Yablonovitch. High-impedance electromagnetic surfaces with a forbidden frequency band. IEEE Transactions on Microwave Theory and Techniques, 47(11):2059-2074, 1999.

[137] Sheng Liu, Michael B. Sinclair, Thomas S. Mahony, Young Chul Jun, Salvatore Campione, James Ginn, Daniel A. Bender, Joel R. Wendt, Jon F. Ihlefeld, Paul G. Clem, Jeremy B. Wright, and Igal Brener. Optical magnetic mirrors without metals. Optica, 1(4):250, 102014.

[138] A S Schwanecke, V A Fedotov, V V Khardikov, S L Prosvirnin, Y Chen, and N I Zheludev. Optical magnetic mirrors. Journal of Optics A: Pure and Applied Optics, 9(1):L1, 112007.

[139] Optical Multilayer Simulator - Lumerical STACK K. https://www.lumerical.com/products/stack/.

[140] I. H. Malitson. Interspecimen Comparison of the Refractive Index of Fused Silica. Journal of the Optical Society of America, 55(10):1205, 101965.

[141] S. Singh, J. R. Potopowicz, L. G. Van Uitert, and S. H. Wemple. Nonlinear optical properties of hexagonal silicon carbide. Applied Physics Letters, 19(3):53-56, 81971.

[142] Mahmoud Hamdy Elshorbagy, Alexander Cuadrado, Gabriel Gonzalez, Francisco Javier Gonzalez, and Javier Alda. Performance Improvement of Refracto- 
metric Sensors Through Hybrid Plasmonic-Fano Resonances. Journal of Lightwave Technology, 37(13):2905-2913, 72019.

[143] Wei Su, Yimin Ding, Yinlong Luo, and Yan Liu. A high figure of merit refractive index sensor based on Fano resonance in all-dielectric metasurface. Results in Physics, 16:102833, 32020.

[144] Peter Offermans, Martijn C. Schaafsma, Said R.K. Rodriguez, Yichen Zhang, Mercedes Crego-Calama, Sywert H. Brongersma, and Jaime Gómez Rivas. Universal scaling of the figure of merit of plasmonic sensors. ACS Nano, 5(6):51515157, 62011.

[145] Jessamine Ng Lee, Cheolmin Park, and George M Whitesides. Solvent Compatibility of Poly(dimethylsiloxane)-Based Microfluidic Devices. Analytical Chemistry, 75(23):6544-6554, 2003.

[146] Wolfgang Mönch, Jan Dehnert, Ewald Jaufmann, and Hans Zappe. FloryHuggins swelling of polymer Bragg mirrors. Applied Physics Letters, 89(16):164104, 2006.

[147] T. H.N. Dinh, E. Martincic, E. Dufour-Gergam, and P. Y. Joubert. Mechanical characterization of PDMS films for the optimization of polymer based flexible capacitive pressure microsensors. Journal of Sensors, 2017, 2017.

[148] L. D. Miranda, R. J. Bell, R. T. Short, F. H.W. van Amerom, and R. H. Byrne. The influence of hydrostatic pressure on gas diffusion in polymer and nano-composite membranes: Application to membrane inlet mass spectrometry. Journal of Membrane Science, 385-386(1):49-56, 122011.

[149] Abel L. Thangawng, Rodney S. Ruoff, Melody A. Swartz, and Matthew R. Glucksberg. An ultra-thin PDMS membrane as a bio/micro-nano interface: Fabrication and characterization. Biomedical Microdevices, 9(4):587-595, 8 2007. 
[150] Zhaoxin Geng, Qing Li, Wei Wang, and Zhihong Li. Theoretical analysis and fabrication of PDMS-based surface plasmon resonance sensor chips. In 2009 4th IEEE International Conference on Nano/Micro Engineered and Molecular Systems, pages 51-54, 2009.

[151] J C Lötters, W Olthuis, P H Veltink, and P Bergveld. The mechanical properties of the rubber elastic polymer polydimethylsiloxane for sensor applications. Journal of Micromechanics and Microengineering, 7(3):145-147, 1997.

[152] Yu Chung Lin, Wen Hsin Hsieh, Lai Kwan Chau, and Guo En Chang. Intensitydetection-based guided-mode-resonance optofluidic biosensing system for rapid, low-cost, label-free detection. Sensors and Actuators, B: Chemical, 250:659-666, 102017.

[153] Nanna B. Hartmann, Thorsten Hüffer, Richard C. Thompson, Martin Hassellöv, Anja Verschoor, Anders E. Daugaard, Sinja Rist, Therese Karlsson, Nicole Brennholt, Matthew Cole, Maria P. Herrling, Maren C. Hess, Natalia P. Ivleva, Amy L. Lusher, and Martin Wagner. Are We Speaking the Same Language? Recommendations for a Definition and Categorization Framework for Plastic Debris. Environmental Science \& Technology, 53(3), 22019.

[154] Robert C. Hale, Meredith E. Seeley, Mark J. La Guardia, Lei Mai, and Eddy Y. Zeng. A Global Perspective on Microplastics, 12020.

[155] GESAMP and ed. Kershaw, P. J. Sources, fate and effects of microplastics in the marine environment: A global assessment. Technical report, IMO/FAO/UNESCO-IOC/UNIDO/WMO/IAEA/UN/UNEP/UNDP Joint Group of Experts on the Scientific Aspects of Marine Environmental Protection, 2015.

[156] Gábor Bordós, Béla Urbányi, Adrienn Micsinai, Balázs Kriszt, Zoltán Palotai, István Szabó, Zsolt Hantosi, and Sándor Szoboszlay. Identification of microplastics in fish ponds and natural freshwater environments of the Carpathian basin, Europe. Chemosphere, 216:110-116, 22019. 
[157] Elke Kerstin Fischer, Lisa Paglialonga, Elisa Czech, and Matthias Tamminga. Microplastic pollution in lakes and lake shoreline sediments - A case study on Lake Bolsena and Lake Chiusi (central Italy). Environmental Pollution, 213:648-657, 62016.

[158] Jingyi Li, Huihui Liu, and J. Paul Chen. Microplastics in freshwater systems: A review on occurrence, environmental effects, and methods for microplastics detection, 62018.

[159] S. Frei, S. Piehl, B. S. Gilfedder, M. G.J. Löder, J. Krutzke, L. Wilhelm, and C. Laforsch. Occurence of microplastics in the hyporheic zone of rivers. Scientific Reports, 9(1):1-11, 122019.

[160] Zhong Pan, Huige Guo, Hongzhe Chen, Sumin Wang, Xiuwu Sun, Qingping Zou, Yuanbiao Zhang, Hui Lin, Shangzhan Cai, and Jiang Huang. Microplastics in the Northwestern Pacific: Abundance, distribution, and characteristics. Science of the Total Environment, 650:1913-1922, 22019.

[161] Thomas Maes, Myra D. Van der Meulen, Lisa I. Devriese, Heather A. Leslie, Arnaud Huvet, Laura Frère, Johan Robbens, and A. Dick Vethaak. Microplastics Baseline Surveys at the Water Surface and in Sediments of the North-East Atlantic. Frontiers in Marine Science, 4(MAY):135, 52017.

[162] Lucy C. Woodall, Anna Sanchez-Vidal, Miquel Canals, Gordon L.J. Paterson, Rachel Coppock, Victoria Sleight, Antonio Calafat, Alex D. Rogers, Bhavani E. Narayanaswamy, and Richard C. Thompson. The deep sea is a major sink for microplastic debris. Royal Society Open Science, 1(4), 122014.

[163] K. L. Law and R. C. Thompson. Microplastics in the seas. Science, 345(6193), 72014.

[164] Christian Baresel and Mikael Olshammar. On the Importance of Sanitary Sewer Overflow on the Total Discharge of Microplastics from Sewage Water. Journal of Environmental Protection, 10(09):1105-1118, 2019. 
[165] Gerald Schernewski, Hagen Radtke, Rahel Hauk, Christian Baresel, Mikael Olshammar, Robert Osinski, and Sonja Oberbeckmann. Transport and Behavior of Microplastics Emissions From Urban Sources in the Baltic Sea. Frontiers in Environmental Science, 8, 102020.

[166] Dafne Eerkes-Medrano, Heather A. Leslie, and Brian Quinn. Microplastics in drinking water: A review and assessment, 22019.

[167] Marcus Eriksen, Laurent C M Lebreton, Henry S Carson, Martin Thiel, Charles J Moore, Jose C Borerro, Francois Galgani, Peter G Ryan, and Julia Reisser. Plastic Pollution in the World's Oceans: More than 5 Trillion Plastic Pieces Weighing over 250,000 Tons Afloat at Sea. PLoS ONE, 9(12):e111913, 2014.

[168] Shaoliang Zhang, Jiuqi Wang, Xu Liu, Fengjuan Qu, Xueshan Wang, Xinrui Wang, Yu Li, and Yankun Sun. Microplastics in the environment: A review of analytical methods, distribution, and biological effects, 22019.

[169] Katsiaryna Pabortsava and Richard S. Lampitt. High concentrations of plastic hidden beneath the surface of the Atlantic Ocean. Nature Communications, 11(1):1-11, 122020.

[170] Erik Van Sebille, Chris Wilcox, Laurent Lebreton, Nikolai Maximenko, Britta Denise Hardesty, Jan A. Van Franeker, Marcus Eriksen, David Siegel, Francois Galgani, and Kara Lavender Law. A global inventory of small floating plastic debris. Environmental Research Letters, 10(12):124006, 122015.

[171] Marcus Eriksen, Nikolai Maximenko, Martin Thiel, Anna Cummins, Gwen Lattin, Stiv Wilson, Jan Hafner, Ann Zellers, and Samuel Rifman. Plastic pollution in the South Pacific subtropical gyre. Marine Pollution Bulletin, 68(1-2):71-76, 32013.

[172] Valeria Hidalgo-Ruz, Lars Gutow, Richard C. Thompson, and Martin Thiel. Microplastics in the marine environment: A review of the methods used for identi- 
fication and quantification. Environmental Science and Technology, 46(6):30603075, 32012 .

[173] Frederic Gallo, Cristina Fossi, Roland Weber, David Santillo, Joao Sousa, Imogen Ingram, Angel Nadal, and Dolores Romano. Marine litter plastics and microplastics and their toxic chemicals components: the need for urgent preventive measures, 122018.

[174] João Pinto da Costa, Vanessa Reis, Ana Paço, Mónica Costa, Armando C. Duarte, and Teresa Rocha-Santos. Micro(nano)plastics - Analytical challenges towards risk evaluation, 22019.

[175] Samaneh Karbalaei, Parichehr Hanachi, Tony R. Walker, and Matthew Cole. Occurrence, sources, human health impacts and mitigation of microplastic pollution, 122018.

[176] A. J. Underwood, M. G. Chapman, and Mark Anthony Browne. Some problems and practicalities in design and interpretation of samples of microplastic waste. Analytical Methods, 9(9):1332-1345, 32017.

[177] ASTM D8332-20, Standard Practice for Collection of Water Samples with High, Medium, or Low Suspended Solids for Identification and Quantification of Microplastic Particles and Fibers. Technical report, ASTM International, West Conshohocken, PA, 2020.

[178] ASTM D8333-20, Standard Practice for Preparation of Water Samples with High, Medium, or Low Suspended Solids for Identification and Quantification of Microplastic Particles and Fibers Using Raman Spectroscopy, IR Spectroscopy, or Pyrolysis-GC/MS. Technical report, ASTM International, West Conshohocken, PA, 2020.

[179] Eoghan M Cunningham and Julia D Sigwart. Environmentally Accurate Microplastic Levels and Their Absence from Exposure Studies. Integrative and Comparative Biology, 59(6), 122019. 
[180] Penelope K. Lindeque, Matthew Cole, Rachel L. Coppock, Ceri N. Lewis, Rachael Z. Miller, Andrew J.R. Watts, Alice Wilson-McNeal, Stephanie L. Wright, and Tamara S. Galloway. Are we underestimating microplastic abundance in the marine environment? A comparison of microplastic capture with nets of different mesh-size. Environmental Pollution, 265, 102020.

[181] Jennifer A. Brandon, Alexandra Freibott, and Linsey M. Sala. Patterns of suspended and salp-ingested microplastic debris in the North Pacific investigated with epifluorescence microscopy. Limnology and Oceanography Letters, 5(1), 2 2020.

[182] Joana Correia Prata, João P. da Costa, Armando C. Duarte, and Teresa RochaSantos. Methods for sampling and detection of microplastics in water and sediment: A critical review. TrAC - Trends in Analytical Chemistry, 110:150159, 12019 .

[183] Benjamin O. Asamoah, Emilia Uurasjärvi, Jukka Räty, Arto Koistinen, Matthieu Roussey, and Kai Erik Peiponen. Towards the development of portable and in situ optical devices for detection of micro and nanoplastics in water: A review on the current status, 32021.

[184] Horst Conrad and Martin E. Kordesch. High Resolution Electron Energy Loss Spectroscopy, Applications. In Encyclopedia of Spectroscopy and Spectrometry, pages 772-783. Elsevier, 11999.

[185] Andreas Lenshof, Cecilia Magnusson, and Thomas Laurell. Acoustofluidics 8: Applications of acoustophoresis in continuous flow microsystems. Lab on a Chip, 12(7):1210-1223, 42012.

[186] Collin D. Davies and Richard M. Crooks. Focusing, sorting, and separating microplastics by serial faradaic ion concentration polarization. Chemical Science, 11(21):5547-5558, 62020. 
[187] Beckett C Colson and Anna P M Michel. Flow-Through Quantification of Microplastics Using Impedance Spectroscopy. ACS Sensors, 2021.

[188] Edward J. Carpenter, Susan J. Anderson, George R. Harvey, Helen P. Miklas, and Bradford B. Peck. Polystyrene spherules in coastal waters. Science, 178(4062):749-750, 111972.

[189] Yukie Mato, Tomohiko Isobe, Hideshige Takada, Haruyuki Kanehiro, Chiyoko Ohtake, and Tsuguchika Kaminuma. Plastic resin pellets as a transport medium for toxic chemicals in the marine environment. Environmental Science and Technology, 35(2):318-324, 12001.

[190] Hrissi K. Karapanagioti and Irene Klontza. Testing phenanthrene distribution properties of virgin plastic pellets and plastic eroded pellets found on Lesvos island beaches (Greece). Marine Environmental Research, 65(4):283-290, 5 2008.

[191] Elke Fries, Jens H. Dekiff, Jana Willmeyer, Marie Theres Nuelle, Martin Ebert, and Dominique Remy. Identification of polymer types and additives in marine microplastic particles using pyrolysis-GC/MS and scanning electron microscopy. Environmental Sciences: Processes and Impacts, 15(10):1949-1956, 102013.

[192] Marten Fischer and Barbara M. Scholz-Böttcher. Simultaneous Trace Identification and Quantification of Common Types of Microplastics in Environmental Samples by Pyrolysis-Gas Chromatography-Mass Spectrometry. Environmental Science and Technology, 51(9):5052-5060, 52017.

[193] Kazuko Matsui, Takahisa Ishimura, Marco Mattonai, Itsuko Iwai, Atsushi Watanabe, Norio Teramae, Hajime Ohtani, and Chuichi Watanabe. Identification algorithm for polymer mixtures based on $\mathrm{Py}-\mathrm{GC} / \mathrm{MS}$ and its application for microplastic analysis in environmental samples. Journal of Analytical and Applied Pyrolysis, 149:104834, 82020. 
[194] Francisca Ribeiro, Elvis D. Okoffo, Jake W. O'Brien, Sarah Fraissinet-Tachet, Stacey O'Brien, Michael Gallen, Saer Samanipour, Sarit Kaserzon, Jochen F. Mueller, Tamara Galloway, and Kevin V. Thomas. Quantitative Analysis of Selected Plastics in High-Commercial-Value Australian Seafood by Pyrolysis Gas Chromatography Mass Spectrometry. Environmental Science and Technology, 54(15):9408-9417, 82020.

[195] Roland Becker, Korinna Altmann, Thomas Sommerfeld, and Ulrike Braun. Quantification of microplastics in a freshwater suspended organic matter using different thermoanalytical methods - outcome of an interlaboratory comparison. Journal of Analytical and Applied Pyrolysis, 148:104829, 62020.

[196] Xiangnan Zhang, Hong Zhang, Kai Yu, Na Li, Yuning Liu, Xiangyu Liu, Hengnan Zhang, Bin Yang, Wenhai Wu, Jing Gao, and Jie Jiang. Rapid Monitoring Approach for Microplastics Using Portable Pyrolysis-Mass Spectrometry. Analytical Chemistry, 92(6):4656-4662, 32020.

[197] Jun Li Xu, Kevin V. Thomas, Zisheng Luo, and Aoife A. Gowen. FTIR and Raman imaging for microplastics analysis: State of the art, challenges and prospects. TrAC - Trends in Analytical Chemistry, 119:115629, 102019.

[198] Gerrit Renner, Torsten C. Schmidt, and Jürgen Schram. Analytical methodologies for monitoring micro(nano)plastics: Which are fit for purpose? Current Opinion in Environmental Science and Health, 1:55-61, 22018.

[199] Anna P. M. Michel, Alexandra Morrison, Victoria Preston, Charles Marx, Beckett Colson, and Helen White. Rapid Identification of Marine Plastic Debris via Spectroscopic Techniques and Machine Learning Classifiers. Environmental Science $\&$ Technology, 72020.

[200] Philipp M. Anger, Elisabeth von der Esch, Thomas Baumann, Martin Elsner, Reinhard Niessner, and Natalia P. Ivleva. Raman microspectroscopy as a tool for microplastic particle analysis. TrAC - Trends in Analytical Chemistry, 109:214-226, 122018. 
[201] Catarina F. Araujo, Mariela M. Nolasco, Antonio M.P. Ribeiro, and Paulo J.A. Ribeiro-Claro. Identification of microplastics using Raman spectroscopy: Latest developments and future prospects. Water Research, 142:426-440, 102018.

[202] Liron Zada, Heather A. Leslie, A. Dick Vethaak, Gerjen H. Tinnevelt, Jeroen J. Jansen, Johannes F. de Boer, and Freek Ariese. Fast microplastics identification with stimulated Raman scattering microscopy. Journal of Raman Spectroscopy, 49(7):1136-1144, 72018.

[203] Chunmao Zhu, Yugo Kanaya, Masashi Tsuchiya, Ryota Nakajima, Hidetaka Nomaki, Tomo Kitahashi, and Katsunori Fujikura. Optimization of a hyperspectral imaging system for rapid detection of microplastics down to $100 \mu \mathrm{m}$. MethodsX, 8:101175, 12021.

[204] Silvia Serranti, Roberta Palmieri, Giuseppe Bonifazi, and Andrés Cózar. Characterization of microplastic litter from oceans by an innovative approach based on hyperspectral imaging. Waste Management, 76, 62018.

[205] Jiajia Shan, Junbo Zhao, Yituo Zhang, Lifen Liu, Fengchang Wu, and Xue Wang. Simple and rapid detection of microplastics in seawater using hyperspectral imaging technology. Analytica Chimica Acta, 1050, 32019.

[206] Silvia Serranti, Ludovica Fiore, Giuseppe Bonifazi, Ayaka Takeshima, Hisato Takeuchi, and Shosaku Kashiwada. Microplastics characterization by hyperspectral imaging in the SWIR range. In Christopher R. Valenta and Masafumi Kimata, editors, SPIE Future Sensing Technologies. SPIE, 112019.

[207] Erkinjon G. Nazarov, Timothy Postlethwaite, Kenneth Markoski, Sophia Koo, and Jeffrey T. Borenstein. Planar Differential Mobility Spectrometry for Clinical Breath Diagnostics. In Diagnostic Devices with Microfluidics. CRC Press, 62017. 
[208] Abraham Vázquez-Guardado, Mason Money, Nathaniel McKinney, and Debashis Chanda. Multi-spectral infrared spectroscopy for robust plastic identification. Applied Optics, 54(24):7396, 82015.

[209] Christina E. Viviano-Beck, Frank P. Seelos, Scott L. Murchie, Eliezer G. Kahn, Kimberley D. Seelos, Howard W. Taylor, Kelly Taylor, Bethany L. Ehlmann, Sandra M. Wisemann, John F. Mustard, and M. Frank Morgan. Revised CRISM spectral parameters and summary products based on the currently detected mineral diversity on Mars. Journal of Geophysical Research E: Planets, 119(6):1403-1431, 62014.

[210] Michaela De Giglio, Marco Dubbini, Irene Cortesi, Michela Maraviglia, Erica Isabella Parisi, and Grazia Tucci. Plastics waste identification in river ecosystems by multispectral proximal sensing: a preliminary methodology study. Water and Environment Journal, page wej.12652, 112020.

[211] Won Joon Shim, Young Kyoung Song, Sang Hee Hong, and Mi Jang. Identification and quantification of microplastics using Nile Red staining. Marine Pollution Bulletin, 113(1-2):469-476, 122016.

[212] Matthew Cole. A novel method for preparing microplastic fibers. Scientific Reports, 6(1):1-7, 102016.

[213] Thomas Maes, Rebecca Jessop, Nikolaus Wellner, Karsten Haupt, and Andrew G. Mayes. A rapid-screening approach to detect and quantify microplastics based on fluorescent tagging with Nile Red. Scientific Reports, 7(1):1-10, 32017.

[214] Gabriel Erni-Cassola, Matthew I. Gibson, Richard C. Thompson, and Joseph A. Christie-Oleza. Lost, but Found with Nile Red: A Novel Method for Detecting and Quantifying Small Microplastics (1 mm to $20 \mu \mathrm{m}$ ) in Environmental Samples. Environmental Science and Technology, 51(23):13641-13648, 122017. 
[215] Camila Q.V. Costa, Joana Cruz, Jorge Martins, Maria Alexandra A. Teodósio, Steffen Jockusch, V. Ramamurthy, and José P. Da Silva. Fluorescence sensing of microplastics on surfaces. Environmental Chemistry Letters, 19(2):1797-1802, 12021.

[216] Thomas Stanton, Matthew Johnson, Paul Nathanail, Rachel L. Gomes, Teresa Needham, and Amanda Burson. Exploring the Efficacy of Nile Red in Microplastic Quantification: A Costaining Approach. Environmental Science and Technology Letters, 6(10):606-611, 102019.

[217] Raymond Gillibert, Gireeshkumar Balakrishnan, Quentin Deshoules, Morgan Tardivel, Alessandro Magazzù, Maria Grazia Donato, Onofrio M. Maragò, Marc Lamy De La Chapelle, Florent Colas, Fabienne Lagarde, and Pietro G. Gucciardi. Raman tweezers for small microplastics and nanoplastics identification in seawater. Environmental Science and Technology, 53(15):9003-9013, 82019.

[218] Christian Schwaferts, Vanessa Sogne, Roland Welz, Florian Meier, Thorsten Klein, Reinhard Niessner, Martin Elsner, and Natalia P. Ivleva. Nanoplastic Analysis by Online Coupling of Raman Microscopy and Field-Flow Fractionation Enabled by Optical Tweezers. Analytical Chemistry, 92(8):5813-5820, 4 2020.

[219] Mohammad Soltani, Jun Lin, Robert A Forties, James T Inman, Summer N Saraf, Robert M Fulbright, Michal Lipson, and Michelle D Wang. Nanophotonic trapping for precise manipulation of biomolecular arrays. 2014.

[220] Domna G. Kotsifaki and Síle Nic Chormaic. Plasmonic optical tweezers based on nanostructures: Fundamentals, advances and prospects. Nanophotonics, 8(7):1227-1245, 72019.

[221] James E. Baker, Ryan P. Badman, and Michelle D. Wang. Nanophotonic trapping: precise manipulation and measurement of biomolecular arrays. Wiley Interdisciplinary Reviews: Nanomedicine and Nanobiotechnology, 10(1):e1477, 12018. 
[222] Xiaofu Xu, Yuming Dong, Guanghui Wang, Wenxiang Jiao, Zhoufeng Ying, Ho Pui Ho, and Xuping Zhang. Reconfigurable Sorting of Nanoparticles on a Thermal Tuning Silicon Based Optofluidic Chip. IEEE Photonics Journal, 10(1), 22018.

[223] Julien Gigault, Hind El Hadri, Stéphanie Reynaud, Elise Deniau, and Bruno Grassl. Asymmetrical flow field flow fractionation methods to characterize submicron particles: application to carbon-based aggregates and nanoplastics. Analytical and Bioanalytical Chemistry, 409(29):6761-6769, 112017.

[224] Guilaume Greyling and Harald Pasch. Thermal Field-Flow Fractionation of Polymers. Springer International Publishing, Cham, 2019.

[225] Filip Petersson, Lena Åberg, Ann Margret Swärd-Nilsson, and Thomas Laurell. Free flow acoustophoresis: Microfluidic-based mode of particle and cell separation. Analytical Chemistry, 79(14):5117-5123, 72007.

[226] Yoshitake Akiyama, Takatoshi Egawa, Kiyoshi Koyano, and Hiroshi Moriwaki. Acoustic focusing of microplastics in microchannels: A promising continuous collection approach. Sensors and Actuators, B: Chemical, 304, 22020.

[227] Oleh Matviykiv, Tamara Klymkovych, Nataliia Bokla, Mykhailo Lobur, Mykhaylo Melnyk, and Anna Timofiejczuk. Simulation of Acoustophoretic Separation of Microplastic Particles in mkFluidic Lab-chip. In 2020 IEEE 16th International Conference on the Perspective Technologies and Methods in MEMS Design, MEMSTECH 2020 - Proceedings, pages 123-126. Institute of Electrical and Electronics Engineers Inc., 42020.

[228] Vaskar Gnyawali, Eric M. Strohm, Jun-Zhi Wang, Scott S. H. Tsai, and Michael C. Kolios. Simultaneous acoustic and photoacoustic microfluidic flow cytometry for label-free analysis. Scientific Reports, 9(1), 122019. 
[229] Vienna L. Mott. Microplastic Detection Using Impedance Measurement in a Microfluidic Channel, Masters Thesis. Tufts University, Medford, Massachusetts, 22020.

[230] Shulin Bu, Mohammad Towshif Rabbani, and Alexandra Ros. Towards Separating Microplastic Particles with Insulator-Based Dielectrophresis . Flagstaff, Arizona, USA, 7 2020. Dielectrophoresis State of the Art Conference 2020.

[231] Yupan Wu, Yukun Ren, Ye Tao, Likai Hou, and Hongyuan Jiang. HighThroughput Separation, Trapping, and Manipulation of Single Cells and Particles by Combined Dielectrophoresis at a Bipolar Electrode Array. Analytical Chemistry, 90(19):11461-11469, 102018.

[232] Tomo Kitahashi, Hiromi Kayama Watanabe, Masashi Tsuchiya, Hideyuki Yamamoto, and Hiroyuki Yamamoto. A new method for acquiring images of meiobenthic images using the FlowCAM. MethodsX, 5:1330-1335, 12018.

[233] Marcus Eriksen, Martin Thiel, Matt Prindiville, and Tim Kiessling. Microplastic: What are the solutions? In Handbook of Environmental Chemistry, volume 58, pages 273-298. Springer Verlag, 2018.

[234] Amy Lusher, Peter Hollman, and Jeremy Mendoza-Hill. Microplastics in fisheries and aquaculture Status of knowledge on their occurrence and implications for aquatic organisms and food safety. Rome, Italy, 2017. FAO Fisheries and Aquaculture Technical Paper.

[235] SB-1422 California Safe Drinking Water Act: microplastics., 92018. 